\title{
Combining gemcitabine with checkpoint kinase inhibitors to sensitize pancreatic tumors
}

\section{Dissertation}

\author{
for the award of the degree \\ "Doctor of Philosophy (Ph.D)" \\ in the "Molecular Biology of Cells" Program \\ at the Georg August University Göttingen, \\ Faculty of Biology
}

submitted by

Priyanka Saini

born in

New Delhi, India

Göttingen 2014 


\section{PhD Thesis committee:}

Prof. Dr. Matthias Dobbelstein, Faculty of Medicine, University of Göttingen (Reviewer) Prof. Dr. Holger Reichardt, Faculty of Medicine, University of Göttingen (Reviewer) Prof. Dr. Dieter Kube, Faculty of Medicine, University of Göttingen

Date of oral exam: $13^{\text {th }}$ October, 2014 


\section{AFFIDAVIT}

Herewith I declare that I prepared the PhD Thesis: "Combining gemcitabine with checkpoint kinase inhibitors to sensitize pancreatic tumors" on my own and with no other sources and aids than quoted.

Göttingen, 29.08.2014 


\section{ACKNOWLEDGMENTS}

Completion of this project would not be possible without the people around me, who helped in their own way for making this happen. Therefore, I would take this opportunity to thank....

First of all, my supervisor, Prof. Matthias Dobbelstein, for giving me the opportunity to work in his lab. His guidance in this project and motivations during struggling times in lab provided a tremendous support in completion of this project.

My thesis committee members, Prof. Holger Reichardt and Prof. Dieter Kube for their critical judgement about the project, providing ideas and being supportive towards the project. My extended thesis committee members, Prof. Heidi Hahn, Prof. Peter Burfeind and Prof. Lutz Walter for their consideration and time.

Erasmus Mundus, Eurindia, program for the scholarship during major part of my doctoral studies. Göttingen Graduate School for Neurosciences, Biophysics, and Molecular Biosciences (GGNB) for stipend and my supervisor for financial support during the required time of my studies.

GGNB for admitting me into the doctoral program, Molecular bology of Cells, and organizing educational retreats and leisure events.

Yizhu Li and Indira Memet for their hard work and contribution in the project. Dr. Norman Ertych for his help in the project.

All members, present and former, of the Institute of Molecular Oncology. Their help, support and encouragement during tough days has nurtured me personally and professionally. The balanced lab environment has made it easier to carry out my studies here.

Dr. Franziska and Dr. Lena for sharing joys and sorrows, intercultural conversations, help in and outside lab and food evenings.

Dr. Frederick, Dr. Uli and Sonja for sharing the office space and having light talks.

Antje, Cathrin and Daniela for providing technical support. Kamila for lab-related work.

Dr. Xin for sharing experiences and assistance in lab. Dr. Manu and Sai for enlightening talks. Yizhu Li for scientific discussions and witty arguments. 
Veena, Vinita, Ankit and Soham for refreshing talks and laughs during lunch. Lalit for his support and motivation during hard times in lab. Rest of the Indian gang in Göttingen, Heena, Avani, Kundan, Pawan, Sumir and Upasana have always supported me in my endeavors.

My brothers, Navneet Saini and Anurag Saini for being so supportive during the time away from the family.

Last but not the least, my parents, Mr. Mehtab Singh Saini and Mrs. Geeta Saini for their constant support and faith during my studies in a foriegn land. 


\section{Table of Contents}

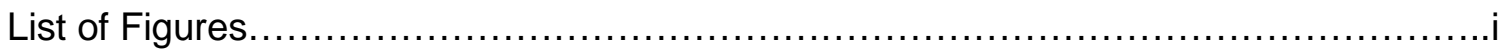

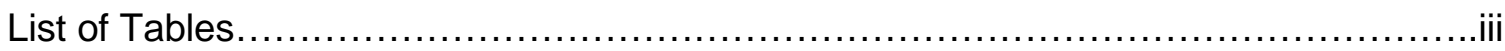

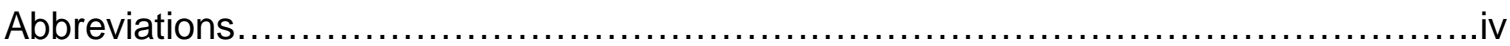

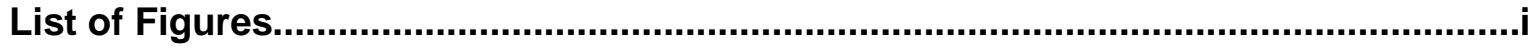

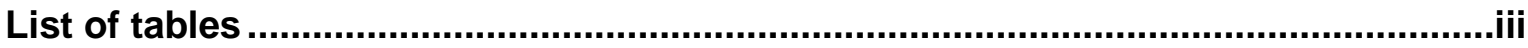

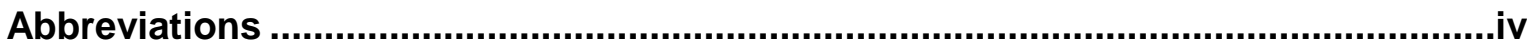

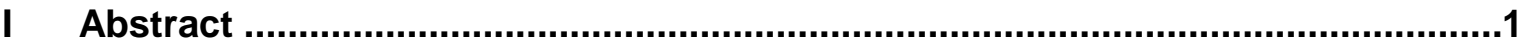

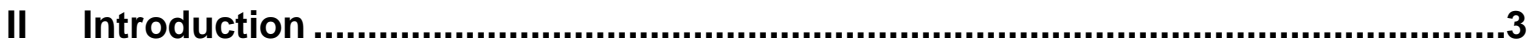

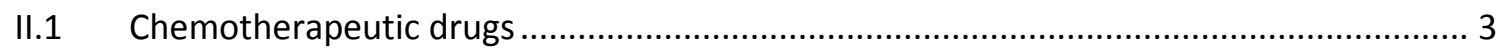

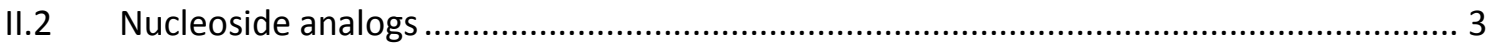

II.3 The cell cycle and its regulation upon DNA damage .................................................. 4

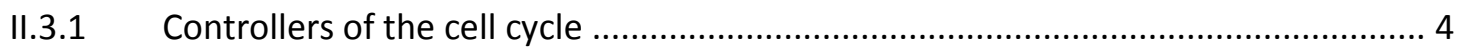

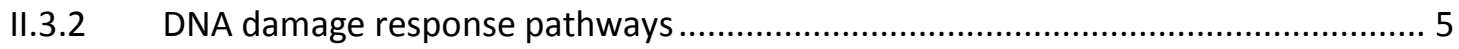

II.3.3 Cyclin-dependent kinases in the DNA damage response ....................................... 9

II.3.4 Checkpoint kinases in cell cycle regulation following DNA damage.......................... 9

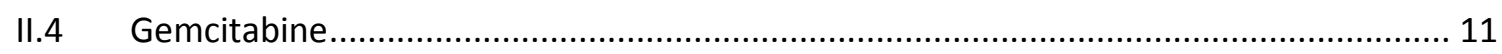

II.4.1 DNA damage response generated by gemcitabine ............................................ 13

II.4.2 Resistance towards gemcitabine in pancreatic cancer......................................... 13

II.5 Targeting cell cycle checkpoint kinases in combination with gemcitabine ..................... 14

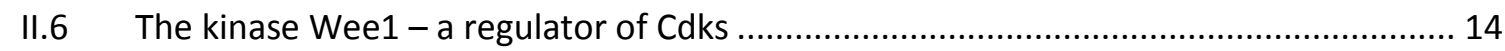

II.7 Polo-like kinase1 and its role in ATR-Chk1 pathway................................................. 17

II.8 Nutlin- 3, as a protector of p53-proficient cells against nucleoside analogues ............... 19

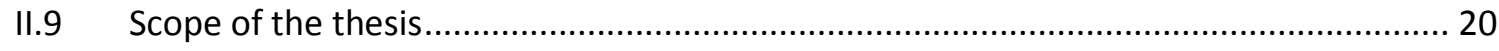

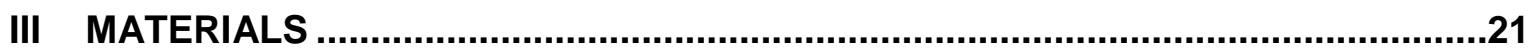

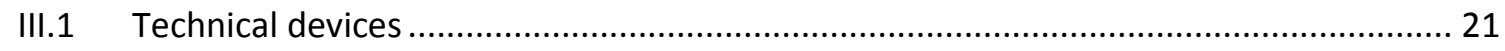

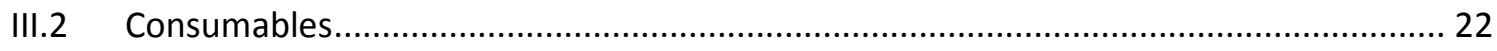

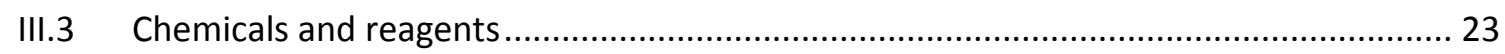

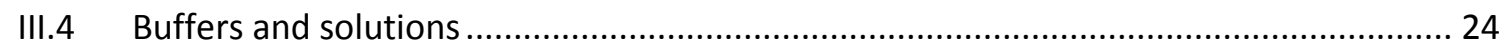

III.5 Chemotherapeutics and pharmacological inhibitor ................................................... 25 


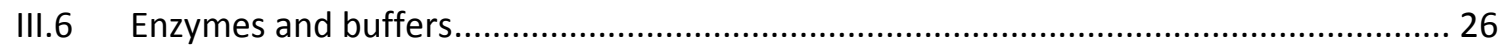

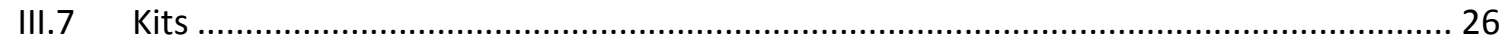

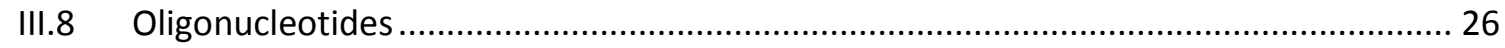

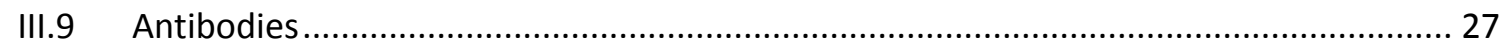

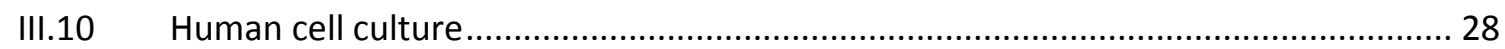

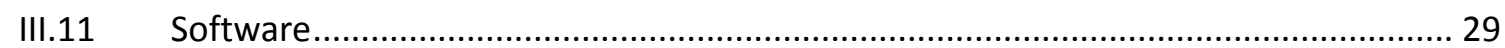

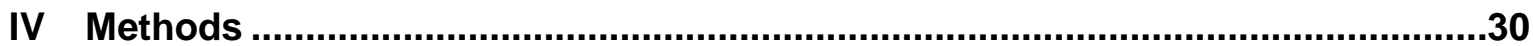

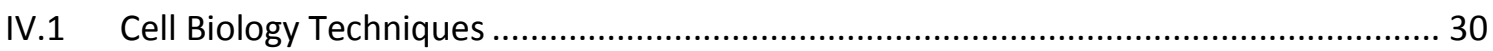

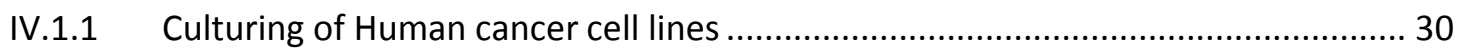

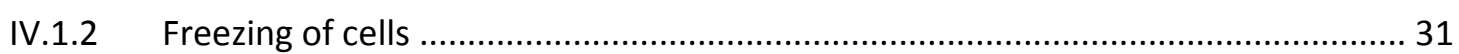

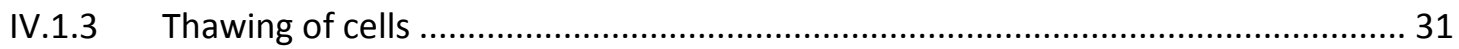

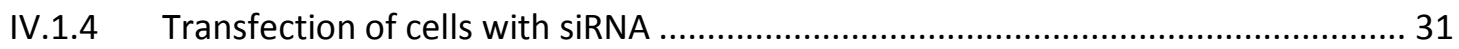

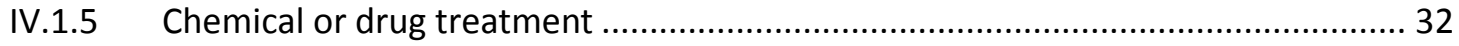

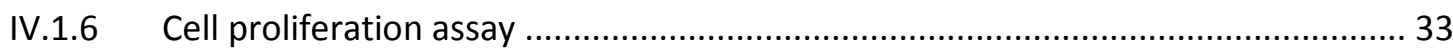

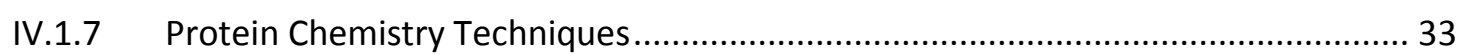

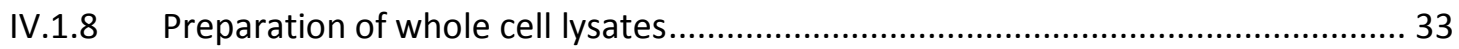

IV.1.9 Separation of proteins by SDS-PAGE (Sodium dodecyl sulfate- Polyacrylamide Gel

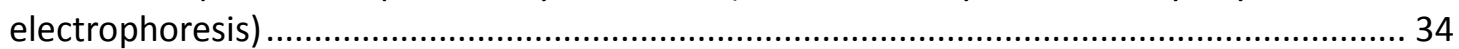

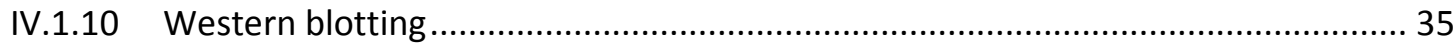

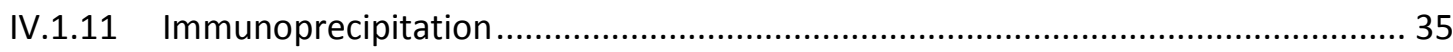

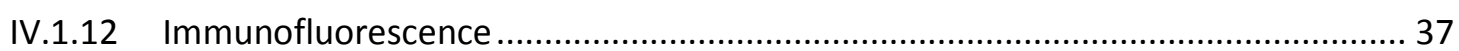

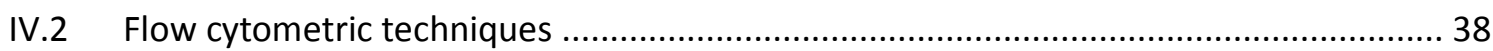

IV.2.1 Cell cycle analysis using Propidium lodide............................................................... 38

IV.2.2 Double thymidine block for cell synchronization ................................................... 39

IV.2.3 Analysis of cells in mitosis or premature mitosis................................................... 39

IV.3 Real Time Quantitative Polymerase Chain Reaction (or qPCR) ...................................... 41

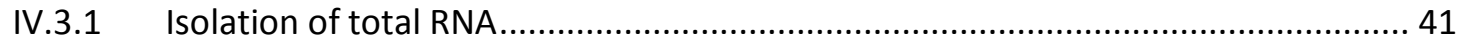

IV.3.2 Conversion of mRNA to cDNA with Reverse transcriptase..................................... 41

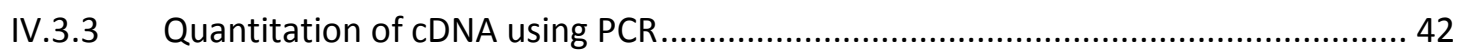

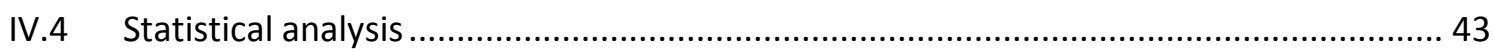

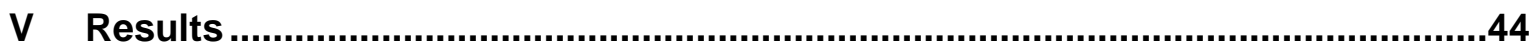

V.1 Chk1, Wee1 and ATR inhibition cooperate with gemcitabine........................................ 44

V.1.1 Chk1, Wee1 or ATR inhibition in combination with gemcitabine intensifies DNA damage response.

V.1.2 Cell growth retards upon combination of Chk1, Wee1 or ATR inhibition with gemcitabine

V.2 Wee1 inhibition inactivates the ATR-Chk1 pathway 
V.2.1 Inhibition or removal of Wee1 in the context of gemcitabine treatment leads to a decrease in Chk1 activation

V.2.2 ATR activation is hampered when Wee1 inhibition is combined with gemcitabine51

V.3 Time-dependent reduction of Chk1 phosphorylation occurs in combination treatment51

V.4 Apoptosis is not the cause of ATR inactivation upon Wee1 inhibition ...................... 53

V.5 Mitotic catastrophe does not lead to down-regulation of ATR-Chk1 pathway............. 53

V.6 Decreased activation of ATR-Chk1 pathway is mediated through Cyclin-dependent kinases 55

V.6.1 Inhibition of Cyclin-dependent kinases (Cdks) using roscovitine restores Chk1 phosphorylation.

V.6.2 Inhibition of Cdk1 could recover Chk1 phosphorylation

V.7 Cdk substrates Mus81 and Retinoblastoma protein do not mediate down-regulation of ATR pathway

V.7.1 Mus81 does not govern inactivation of ATR signaling pathway.....

V.7.2 The Retinoblastoma protein, negatively regulated by Cdks, does not affect the ATR pathway 59

V.8 Polo- like kinase 1 (PIk1) impedes the ATR- Chk1 pathway.

V.8.1 Inhibition of Plk1 recovers decreased Chk1 and Rad17 activation upon Wee1 inhibition and gemcitabine.

V.8.2 Plk1 mediates inactivation of Chk1 through Claspin degradation.

V.9 Nutlin-3 pretreatment attenuates DNA damage response and apoptosis upon Wee1 inhibition with gemcitabine in p53-proficient cells

V.9.1 U2OS, a cell line with wild-type p53, resists cytotoxic effects of combination treatment upon nutlin-3 pretreatment.

V.9.2 Wild-type p53 is required for protective effects of nutlin-3

V.10 Long-term survival of cells treated with Wee1 inhibitor and gemcitabine increases upon pre-treatment with nutlin-3

V.11 Addition of nutlin-3 protects cells from premature mitosis resulting from combination therapy

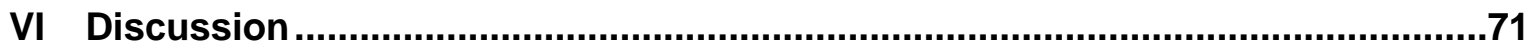

VI.1 Emergence of combination therapy ........................................................... 71

VI.2 Crosstalk between Wee1 and ATR-Chk1 pathway ............................................... 72

VI.3 Decreased activation of ATR-Chk1 pathway is mediated through Cyclin-dependent

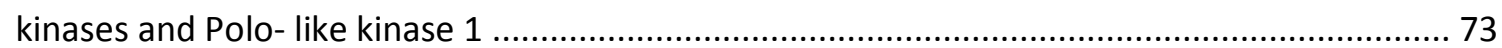

VI.4 Attenuating the side-effects of combination treatment by nutlin-3 pretreatment........ 76

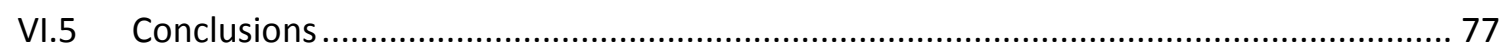

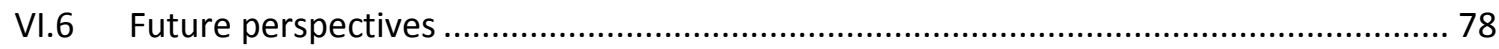

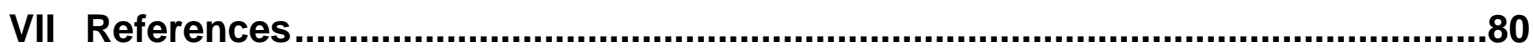

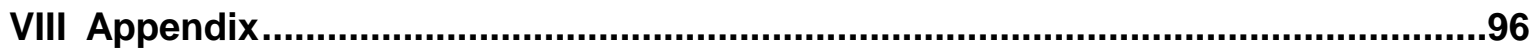




\section{List of Figures}

Figure II-I Oscillation of cyclin-cdk complexes in the cell cycle. ..................................5

Figure II-II Activation of ATR-Chk1 pathway. .........................................................

Figure II-III Checkpoint signaling in response to DNA damage.................................11

Figure II-IV Structures of deoxycytidine and gemcitabine. ........................................12

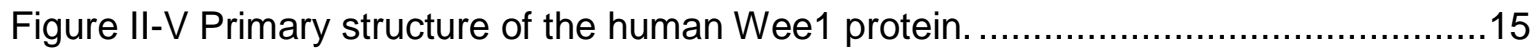

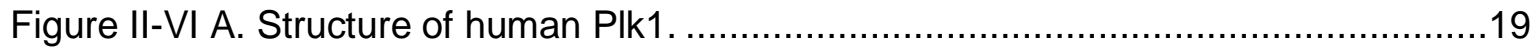

Figure IV-I Flow cytometric analysis of cells in premature mitosis................................40

Figure V-I Three checkpoint inhibitors cooperate with gemcitabine. ............................45

Figure V-II Depletion of the checkpoint kinases sensitize cells towards gemcitabine......47

Figure V-III Long-term survivability of cells decreases upon combination of Wee1 or ATR inhibitor with gemcitabine.

Figure V-IV Wee1 inhibition or knockdown in combination with gemcitabine, reduces Chk1 activation.

Figure V-V ATR activity decreases upon combination of Wee1 inhibitor and gemcitabine.

Figure V-VI Chk1 activity reduces in a time-dependent manner upon combining Wee1

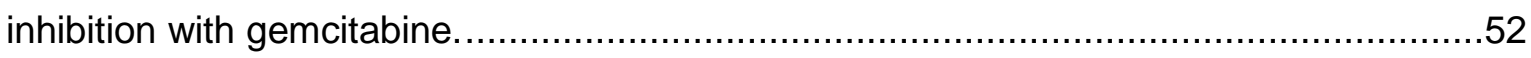

Figure V-VII Caspase activity does not cause loss of Chk1 activation. ..........................53

Figure V-VIII Mitotic catastrophe does not lead to reduction in ATR-Chk1 activity...........55

Figure V-IX Cdk inhibition rescues decreased Chk1 and ATR activity upon Wee1 inhibition with gemcitabine

Figure V-X Cdk1 inhibition recovers Chk1 and Rad17 phosphorylation upon Wee1 inhibition and gemcitabine treatment.

Figure V-XI Mus81 does not mediate ATR-Chk1 inactivation upon Wee1 inhibition and gemcitabine treatment.

Figure V-XII Retinoblastoma protein does not mediate the down-regulation of ATR-Chk1 activity upon inhibition of Wee1 and gemcitabine treatment.

Figure V-XIII Inhibition of Plk1 rescues ATR-Chk1 activity.

Figure V-XIV Plk1 causes Claspin degradation which leads to Chk1 inactivation upon Wee1 inhibition with gemcitabine. .65

Figure V-XV Nutlin-3 attenuates the cytotoxicity caused by combination of Wee1 inhibitor and gemcitabine.

Figure V-XVI p53 is required for protection by nutlin-3. 
Figure V-XVII Pretreatment with nutlin-3 increases long-term survival of the cells treated with Wee1 inhibitor and gemcitabine. .69

Figure V-XVIII Nutlin-3 pretreatment protects cells from premature mitosis caused by

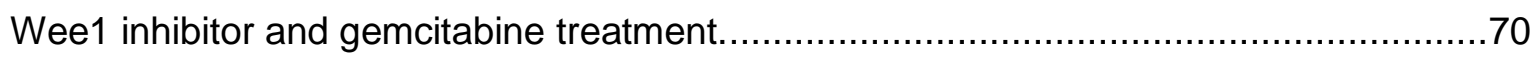

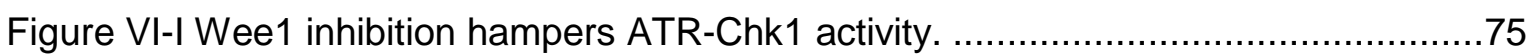

Figure VIII-I Checkpoint kinase inhibitors efficiently inhibit their target kinases...............96

Figure VIII-II Wee1 \#1 siRNA was quite efficient in removing the Wee1 protein. .............96

Figure VIII-III Two different siRNAs against Chk1 are efficient in knockdown of Chk1 protein .97

Figure VIII-IV Cells with Wee1 inhibition in the absence or presence of gemcitabine show entry into premature mitosis. .99 


\section{List of tables}

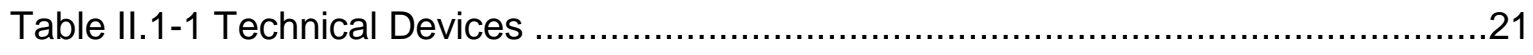

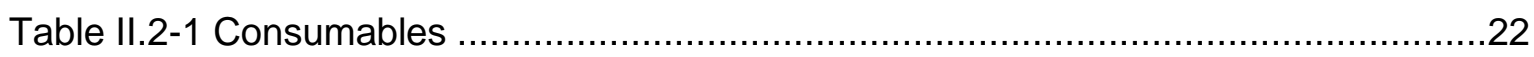

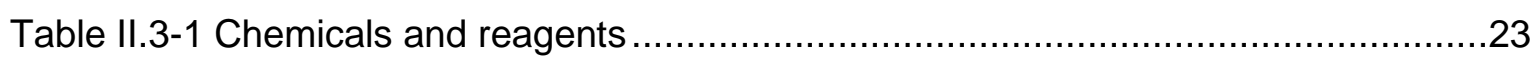

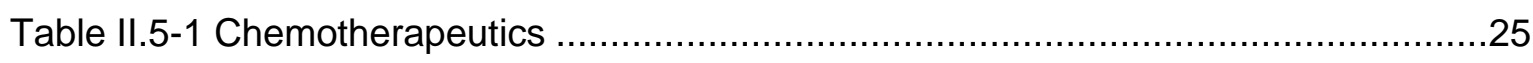

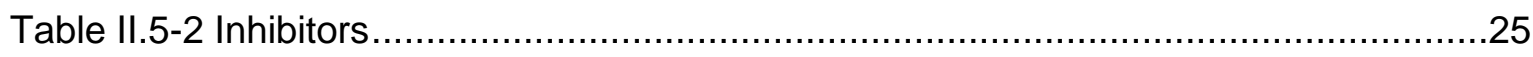

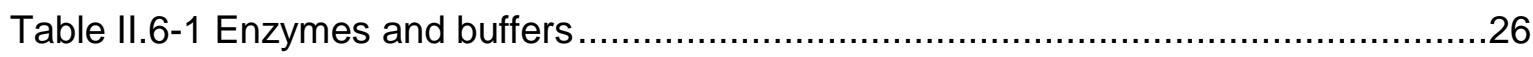

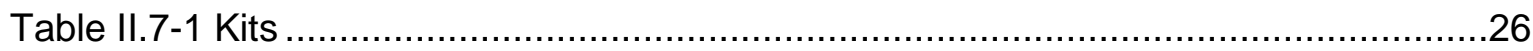

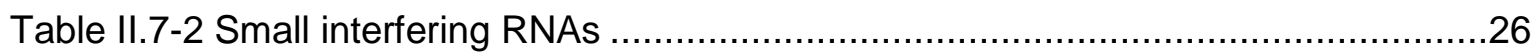

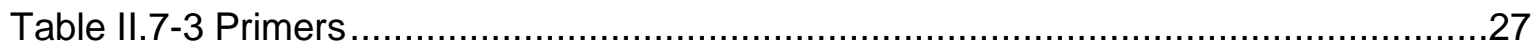

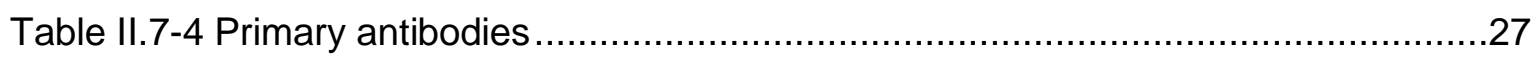

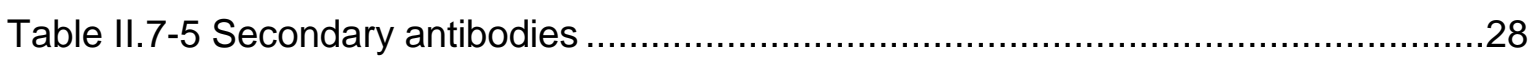

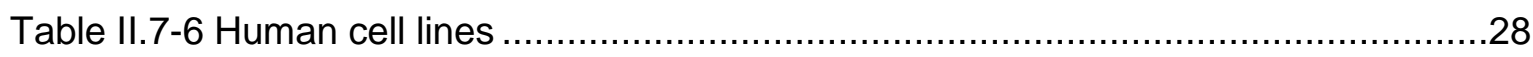

Table II.7-7 Media and reagents for eukaryotic cell culture ....................................28

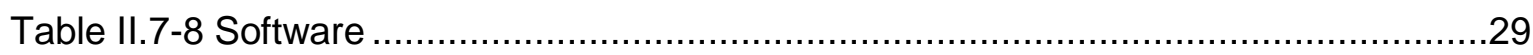

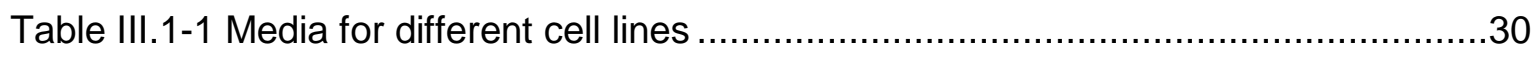

Table III.1-2 siRNA and lipofectamine dilution according to plate format ......................32

Table III.1-3 Concentration of chemicals or drugs used in the treatment ........................32

Table III.1-4 Components of stacking and resolving gels............................................34

Table III.3-1 Reagents required for Reverse Transcription reaction ..............................42

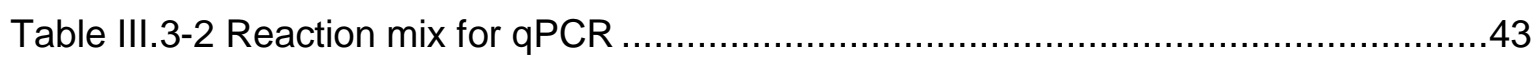

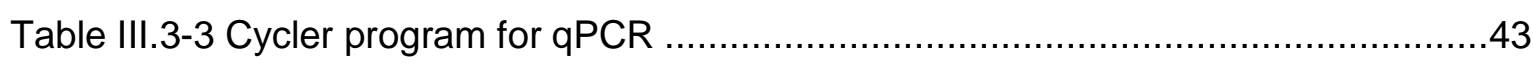




\section{Abbreviations}

\begin{tabular}{|c|c|}
\hline \# & number \\
\hline$\beta$-TrCP1/2 & $\beta$-transducin repeat-containing protein $1 / 2$ \\
\hline${ }^{\circ} \mathrm{C}$ & Degree Celcius \\
\hline$\mu \mathrm{g}$ & Microgram \\
\hline$\mu \mathrm{L}$ & Microliter \\
\hline$\mu \mathrm{M}$ & Micromolar \\
\hline $9-1-1$ & Rad9-Hus1-Rad1 \\
\hline AlF & Apoptosis-inducing Factor \\
\hline APC/C & Anaphase-promoting complex / cyclosome \\
\hline APS & Ammonium persulfate \\
\hline ATM & Ataxia telangiectasia mutated \\
\hline ATP & Adenosine triphosphoate \\
\hline ATR & ATM- and Rad3-related \\
\hline ATRIP & ATR interacting protein \\
\hline bp & Base pair \\
\hline $\mathrm{Bcl}-\mathrm{xl}$ & B-cell lymphoma-extra large \\
\hline BNIP3 & BCL2/Adenovirus E1B 19kDa Interacting Protein 3 \\
\hline BRCA1 & Breast Cancer 1, Early Onset \\
\hline BSA & Bovine serum albumine \\
\hline Cables & CDK5 and ABL1 enzyme substrate 1 \\
\hline CAK & Cdk-activating kinase \\
\hline Cdk & Cyclin-dependent kinase \\
\hline cDNA & Complementary DNA \\
\hline CDT1 & Chromatin Licensing And DNA Replication Factor 1 \\
\hline Chk1 & Checkpoint kinase 1 \\
\hline Chk2 & Checkpoint kinase 2 \\
\hline CKI & Cdk inhibitor \\
\hline $\mathrm{cm}$ & Centimeter \\
\hline C-terminus & Carboxy terminus \\
\hline CtBP & C-terminal binding protein \\
\hline CtIP & CtBP-Interacting Protein \\
\hline CTP & Cytidine triphosphate \\
\hline dCMP & Deoxycytidine monophosphate \\
\hline DDR & DNA damage response \\
\hline DMSO & Dimethylsulfoxide \\
\hline
\end{tabular}




\begin{tabular}{|c|c|}
\hline DNA & Deoxyribonucleic acid \\
\hline DNA-PK & DNA-dependent protein kinase \\
\hline dNTP & Deoxynucleotide triphosphate \\
\hline DSB & Double strand break \\
\hline DTT & Dithiotreitol \\
\hline EDTA & Ethylene diamine tetraacetic acid \\
\hline EGTA & Ethylene glycol tetraacetic acid \\
\hline Eme1 & Essential Meiotic Structure-Specific Endonuclease 1 \\
\hline ERK & Extracellular signal-regulated kinase \\
\hline $\mathrm{EtOH}$ & Ethanol \\
\hline FCS & Fetal calf serum \\
\hline g & Gravitational force \\
\hline $\mathrm{h}$ & Hour \\
\hline $\mathrm{H} 2 \mathrm{AX}$ & Histone variant $2 \mathrm{AX}$ \\
\hline $\mathrm{H} 2 \mathrm{O}$ & water \\
\hline H3 & Histone 3 \\
\hline HDAC & Histone deacetylase \\
\hline hENT1 & Human equilibrative nucleoside transporter 1 \\
\hline HRP & Horseradish peroxidase \\
\hline HSP70 & Heat-shock protein 70 \\
\hline IF & Immunofluorescence \\
\hline $\mathrm{i}$ & Inhibitor \\
\hline IP & Immunoprecipitation \\
\hline IR & Ionizing radiation \\
\hline $\mathrm{kDa}$ & Kilodalton \\
\hline M & Molar \\
\hline MAP kinase & Mitogen-activated protein kinase \\
\hline MC & Mitotic catastrophe \\
\hline MDC1 & Mediator of DNA-damage checkpoint 1 \\
\hline Mdm2 & Mouse Double Minute 2 \\
\hline $\mathrm{mg}$ & Milligram \\
\hline $\min$ & Minute \\
\hline miRNA & Micro RNA \\
\hline $\mathrm{mL}$ & Milliliter \\
\hline $\mathrm{mM}$ & Millimolar \\
\hline MMS & Methyl methanesulfonate \\
\hline MPM-2 & Mitotic Protein Monoclonal \#2 \\
\hline
\end{tabular}




\begin{tabular}{|c|c|}
\hline MRN & MRE11/NBS1/RAD50 \\
\hline mRNA & Messenger RNA \\
\hline Mus81 & MMS and UV-sensitive protein 81 \\
\hline$n$ & Sample size \\
\hline ng & Nanogram \\
\hline $\mathrm{nM}$ & Nanomolar \\
\hline NP-40 & Nonidet $\mathrm{P}-40$ substitute \\
\hline n.s. & Not significant \\
\hline $\mathrm{p}$ & Phospho \\
\hline p53BP1 & p53 binding protein 1 \\
\hline PBD & Polo-box domain \\
\hline PBS & Phosphate buffered saline \\
\hline PCR & Polymerase chain reaction \\
\hline $\mathrm{PHH} 3$ & Phospho-histone 3 \\
\hline PI & Propidium iodide \\
\hline PIKK & Phosphatidylinositol 3-kinase related kinase \\
\hline Plk1 & Polo-like kinase 1 \\
\hline PP2A & Protein phosphatase $2 \mathrm{~A}$ \\
\hline PP4C & Protein phosphatase $4 \mathrm{C}$ \\
\hline $\mathrm{pRb}$ & Retinoblastoma protein \\
\hline qPCR & Quantitative real-time PCR \\
\hline RFC & Replication factor C \\
\hline RNA & Ribonucleic acid \\
\hline ROIs & Region of interest \\
\hline RPA & Replication protein A \\
\hline rpm & Rounds per minute \\
\hline $\mathrm{RR}$ & Ribonucleotide reductase \\
\hline RT & Room temperature; Reverse transcriptase \\
\hline Sae2 & SUMO1 Activating Enzyme Subunit 2 \\
\hline SCF & SKP1/Cul1/F-box protein \\
\hline SDS & Sodium dodecyl sulfate \\
\hline SDS-PAGE & SDS-polyacrylamide gel electrophoresis \\
\hline sec & Second \\
\hline SiRNA & Small interfering ribonucleic acid \\
\hline ssDNA & Single-stranded DNA \\
\hline SLX4 & Synthetic lethal of unknown function protein 4 \\
\hline SMC1 & Structural Maintenance Of Chromosomes \\
\hline
\end{tabular}


Abbreviations

$\begin{array}{ll}\text { TBST } & \text { Tris buffered saline }+ \text { Tween } 20 \\ \text { TEMED } & \text { Tetramethylethylenediamine } \\ \text { TOPBP1 } & \text { DNA topoisomerase2-binding protein1 } \\ \text { TP53 } & \text { Tumor protein p53 } \\ \text { Tris } & \text { Trisamine } \\ \text { UV } & \text { Ultraviolet } \\ \text { V } & \text { Volt } \\ \text { WT } & \text { Wild type } \\ \text { YH2AX } & \text { H2AX phosphorylated on S319 }\end{array}$

Three or one letter codes were used for amino acids. 


\section{Abstract}

Pancreatic tumor is one of the leading causes of cancer-related deaths in the world. Currently, the nucleoside analogue gemcitabine is the leading therapeutic drug for the treatment of pancreatic tumors. However, due to an ever-increasing number of patients developing gemcitabine resistance, there is a renewed interest in developing more efficient treatment regimes.

Combination therapy that utilizes gemcitabine with other chemotherapeutic drugs or biological agents has the potential to overcome issues with traditional gemcitabine therapy. Gemcitabine acts by inducing replicative stress and consequently, cell cycle checkpoint kinases are activated. Tumor cells have more efficient checkpoint control, which could ultimately cause resistance towards gemcitabine. Therefore, inhibitors against checkpoint kinases are attractive candidates for tumor treatment in combination with gemcitabine. In this study, we have evaluated the sensitization of several pancreatic tumor cell lines (Panc1, MiaPaCa2 and BxPC3) towards gemcitabine upon inhibition of Chk1, Wee1 and ATR checkpoint kinases. We find that inhibition of these checkpoint kinases with specific chemical inhibitors sensitize pancreatic tumor cells against gemcitabine. Of these, the combination of Wee1 inhibitor, MK-1775 with gemcitabine shows high efficiency in decreasing the long-term survivability of cells and elimination of pancreatic tumor cells.

Through western blot analysis, we find that Wee1 inhibition along with gemcitabine treatment causes inactivation of the ATR signaling pathway. We show that apoptosis and mitotic catastrophe do not cause the reduction in ATR-Chk1 activity. Interestingly, the attenuation of ATR-Chk1 pathway can be rescued by simultaneous inhibition of Cdks. Surprisingly, we find that simultaneous inhibition of Plk1 along with Wee1 inhibition and gemcitabine treatment can also recover the decreased ATR-Chk1 activity. We observe that activation of Plk1 upon Wee1 inhibition along with gemcitabine is dependent on Cdks. Moreover, we also show that Plk1 mediates inactivation of Chk1 through Claspin degradation.

In order to reduce the toxic effects of the combined treatment of Wee1 inhibitor with gemcitabine in normal proliferating cells with wild-type p53, we tested Mdm2 antagonist, nutlin-3 pretreatment. We find that indeed nutlin-3 pretreatment can decrease the DNA damage response, apoptosis as well as the cells entering into mitosis prematurely caused 
by Wee1 inhibition with gemcitabine. As expected, this virtue of nutlin-3 pretreatment is dependent on p53 status of the cells.

In conclusion, our study shows that the efficiency of Wee1 inhibition and gemcitabine treatment is not solely dependent on cell cycle dysregulation but also on the replicative stress. Since most of the pancreatic tumors have mutated form of $p 53$, we propose that pretreatment with Mdm2 antagonists at sub-lethal dose can provide protection to fast proliferating cells with wild-type p53 against toxic effects of combination of Wee1 inhibition and gemcitabine treatment. 


\section{Introduction}

\section{II.1 Chemotherapeutic drugs}

Cancer is a group of diseases which involve abnormal division of cells and their spreading to other parts of the body. Cancer management involves several procedures including surgery, radiotherapy and chemotherapy. Chemotherapy uses one or more cytotoxic antineoplastic drugs (McKnight 2003). Chemotherapeutic drugs have been classically categorized based on their chemical structure and mechanism of action: Alkylating agents, antibiotics, antimetabolites, topoisomerase I and II inhibitors, mitosis inhibitors, platinum compounds and others (Espinosa et al. 2003). Among these categories of the drugs, antimetabolites comprise the structural analogs of naturally occurring metabolites involved in RNA or DNA synthesis (Malhotra and Perry 2003).

\section{II.2 Nucleoside analogs}

Nucleoside analogs are the antimetabolites which are structurally similar to nucleosides, have a broad range of action, and are clinically active in both solid tumors and hematological malignancies (B Ewald, Sampath, and Plunkett 2008).

\section{II.2.1.1 Classification}

Nucleoside analogs can be sub-categorized into pyrimidine analogs and purine analogs. Pyrimidine analogs are deoxycytidine derivatives and include gemcitabine, ara- $\mathrm{C}$ (or cytarabine), troxacitabine. They get incorporated into the replicating DNA and this is at least one of the major mechanisms of their cytotoxicity, ribonucleotide reductase inhibition is also important in the case of gemcitabine. Likewise, purine analogs are derivatives of deoxyadenosine such as fludarabine, cladrabine, clofarabine. Purine analogs exert their cytotoxic effects by getting incorporated into both DNA and RNA. Furthermore, they can activate DNA-independent processes to promote apoptosis (they change the mitochondrial membrane potential leading to release of cytochrome c; which, in turn, binds to other pro-apoptotic proteins to form an active apoptotic complex called the apoptosome). Purine analogs have been found to be potent in B-cell malignancies while pyrimidine analogs are active in a broad spectrum of solid tumors (Daskalakis et al. 2002, Gore et al. 2006, B Ewald, Sampath, and Plunkett 2008). 


\section{II.2.1.2 Mechanism of action}

The triphosphates of nucleoside analogs compete with natural nucleotides for incorporation into the DNA by DNA polymerases causing steric hindrance to the extending replication forks, thereby leading to fork stalling (Kufe et al. 1980, Huang et al. 1991). As these agents exert their cytotoxic effects after getting incorporated into the DNA, they are predominantly active in cells undergoing active DNA replication or excision repair synthesis (Huang, Chubb, and Plunkett 1990, Yamauchi et al. 2001). Once replication is blocked, cells activate the intra S-phase checkpoint which halts DNA replication and causes S-phase arrest discussed further in section II.3.2 (Shi et al. 2001, Sampath, Shi, and Plunkett 2002, Y.-W. Zhang, Hunter, and Abraham 2006). However, these evolutionary conserved mechanisms of safeguarding the genome are exploited by nucleoside analogs and other DNA-targeting drugs to cause enhanced cell killing. They effectuate cell death either by direct activation of the apoptosome (Genini et al. 2000, Bellosillo et al. 2002, Riedl and Salvesen 2007) or through epigenetic modifications (Stresemann and Lyko 2008).

\section{II.3 The cell cycle and its regulation upon DNA damage}

\section{II.3.1 Controllers of the cell cycle}

Cyclin dependent protein kinases (cdks) are among the major regulators of the cell cycle. Cdks are the catalytic subunits that dimerize with regulatory subunits, cyclins, to get activated. In humans, 11 genes have been shown to encode different Cdks that associate with specific cyclins in a cell cycle dependent manner (Malumbres et al. 2009), and are subsequently phosphorylated by a Cdk-activating kinases (CAKs) to form active complexes (Morgan 1995).

Transition from G0-G1 (in quiescent cells) and early G1 (in proliferating cells) has been found to involve Cdk4-CyclinD, Cdk6-CyclinD and Cdk3-CyclinC complexes (at least in human cells), which mediate their action by phosphorylating the retinoblastoma protein (pRb). Cdk2-CyclinE complex has been proposed to complete the phosphorylation of $\mathrm{pRb}$, therefore, allowing the cells to proceed through the cell cycle. Cdk2-CyclinE complex has also been implicated in the G1-S transition by licensing DNA origins of replication. Cdk2 later associates with CyclinA during progression through the $S$ phase. Cdk1 participates in the S-G2 and G2-M transitions by sequential binding to Cyclin A and Cyclin B (Malumbres and Barbacid 2005). Summarized in Figure II-I. 
$\mathrm{pRb}$ is a tumor suppressor protein that regulates G1-S transition of the cell cycle and differentiation depending on the type of cellular proteins it binds (Taya 1997). Two wellknown substrates of $\mathrm{pRb}$ are E2F and Histone deacetylases (HDACs). pRb interacts with E2F and HDACs forming a trimeric complex that represses transcription of a number of cell cycle regulated proteins (Harbour and Dean 2000).

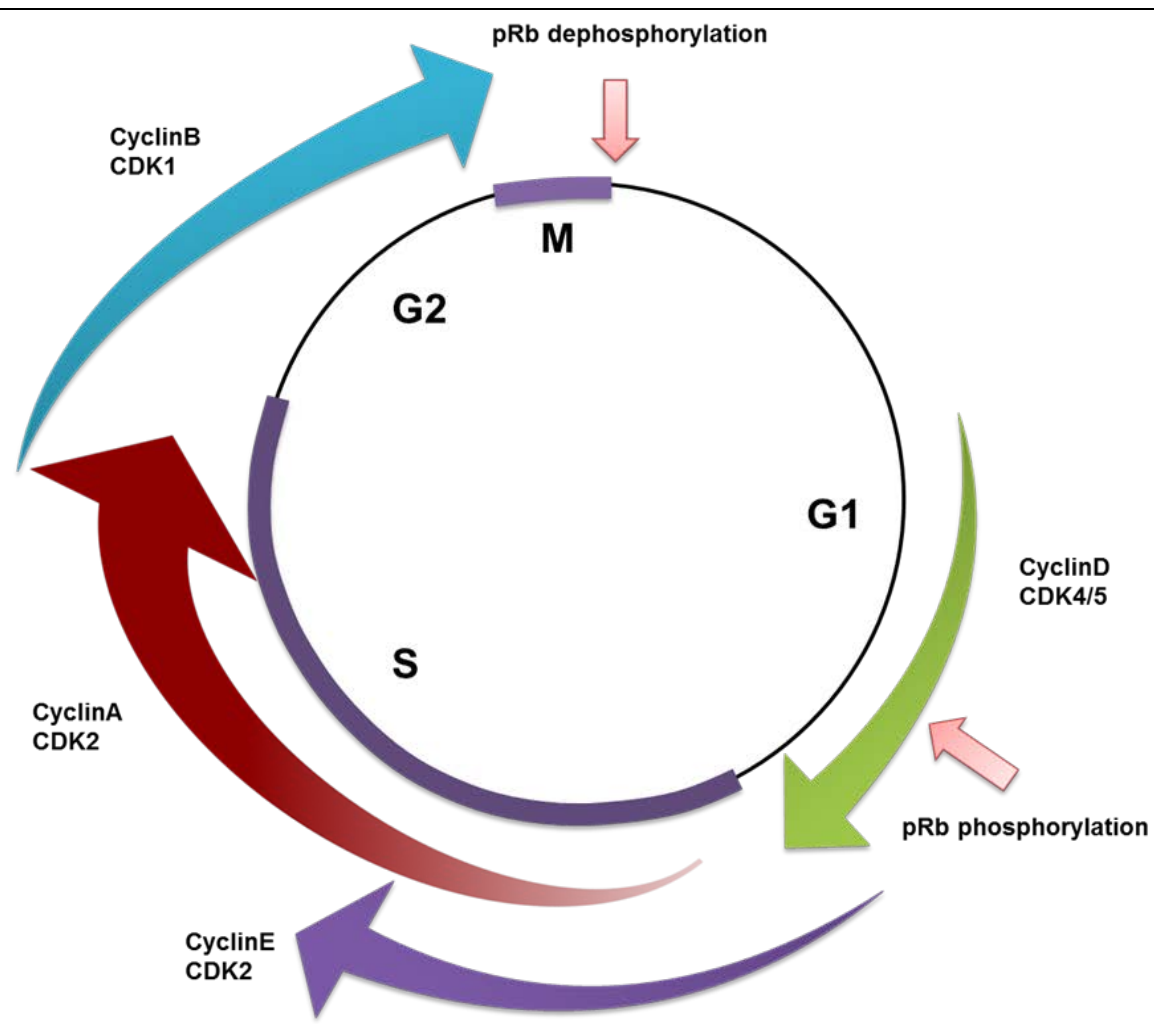

Figure II-I Oscillation of cyclin-cdk complexes in the cell cycle.

Different combinations of cyclin-cdk complexes play role in driving the cell through various phases of the cell cycle.

\section{II.3.2 DNA damage response pathways}

In general, DNA damage or obstruction of DNA replication results in the recruitment of an array of molecular factors that comprise the DNA damage response machinery. These molecular factors bring about the cellular response to the DNA damage either by activating checkpoints, initiating DNA repair or causing cell death. When DNA damage either through UV or replicative stress is encountered by the cells, they respond to it by activating a series of proteins that co-ordinates DNA replication, DNA repair and cell-cycle progression and regulates processes such as firing of replication origins (Santocanale and Diffley 1998, Shirahige et al. 1998, Santocanale, Sharma, and Diffley 1999), stabilization of DNA replication forks in response to DNA damage or replicative stress (Lopes et al. 2001, Tercero and Diffley 2001), resumption of stalled DNA replication forks (Desany et al. 1998, Szyjka et al. 2008), transcriptional induction of DNA damage 
response genes (Allen et al. 1994), choice of the repair pathway (Kai et al. 2007) and inhibition of mitosis until replication is completed (Allen et al. 1994). Central among these are three phosphoinositide 3-kinase-related protein kinases (PIKKs) or serine/threonine kinases- Ataxia-telangiectasia mutated (ATM), Ataxia-telangiectasia mutated and rad3related (ATR) and DNA-dependent protein kinase (DNA-PK) (B Ewald, Sampath, and Plunkett 2008).

\section{II.3.2.1 ATR-Chk1 pathway}

Stalling of replication forks results in single-stranded DNA (sSDNA) that gets coated by replication protein A (RPA), which then recruits ATR. ATR is specific for ssDNA and interacts with ATR interacting protein (ATRIP) which serves as a platform for ATR activation (Cortez et al. 2001). Once ATR is recruited to DNA damage site, it gets autophosphorylated at Thr1989, which is important for its activation (S. Liu et al. 2011). Upon failing to stabilize forks, ATR can activate the apoptotic machinery directly or through Chk1 that phosphorylates and thus, activates p53 (Tibbetts et al. 1999, Shieh et al. 2000). Like ATR, Rad17 is also recruited to the sites of RPA coated single- stranded DNA but independently of ATR (Zou and Elledge 2003, Melo, Cohen, and Toczyski 2001). Rad17, along with the four small subunits of replication factor C (RFC2-5), acts as a clamp loader of Rad9-Rad1-Hus1 (9-1-1) at or near the sites of DNA damage (Zou, Cortez, and Elledge 2002). 9-1-1, in turn, recruits DNA topoisomerase2-binding protein1 (TopBP1) that recognizes auto-phosphorylated site on ATR (Thr1989) and thus, activates it (Delacroix et al. 2007, Akiko Kumagai et al. 2006, J. Lee, Kumagai, and Dunphy 2007). Depicted in Figure II-II.

ATR activates Chk1 by phosphorylating Claspin, thereby creating a docking site for Chk1 and its subsequent phosphorylation by ATR at Ser317 and Ser345 (Akiko Kumagai and Dunphy 2003, (Q. Liu et al. 2000), Guo et al. 2000, (H Zhao and Piwnica-Worms 2001). After ATR-induced phosphorylation, Chk1 undergoes autophosphorylation at Ser296 (Kasahara et al. 2010). Once phosphorylated, Chk1 dissociates from chromatin; thus, ATR regulation of Chk1 may control the transmission of DNA damage signals from chromatin to its targets (Smits, Reaper, and Jackson 2006). Chk1 mediates cell cycle regulation by phosphorylating Cdc25 phosphatase and activating Wee1 that directly inhibit Cyclin-dependent kinases (Cdks) activity by phosphorylation at Tyr 15 (J. Lee, Kumagai, and Dunphy 2001, Rothblum-Oviatt, Ryan, and Piwnica-Worms 2001). It has also been shown that phosphorylation of Rad17 by ATR at Ser635 and Ser645 is required for cells to initiate cell cycle arrest following DNA damage (Bao et al. 2001, Medhurst et al. 2008). 


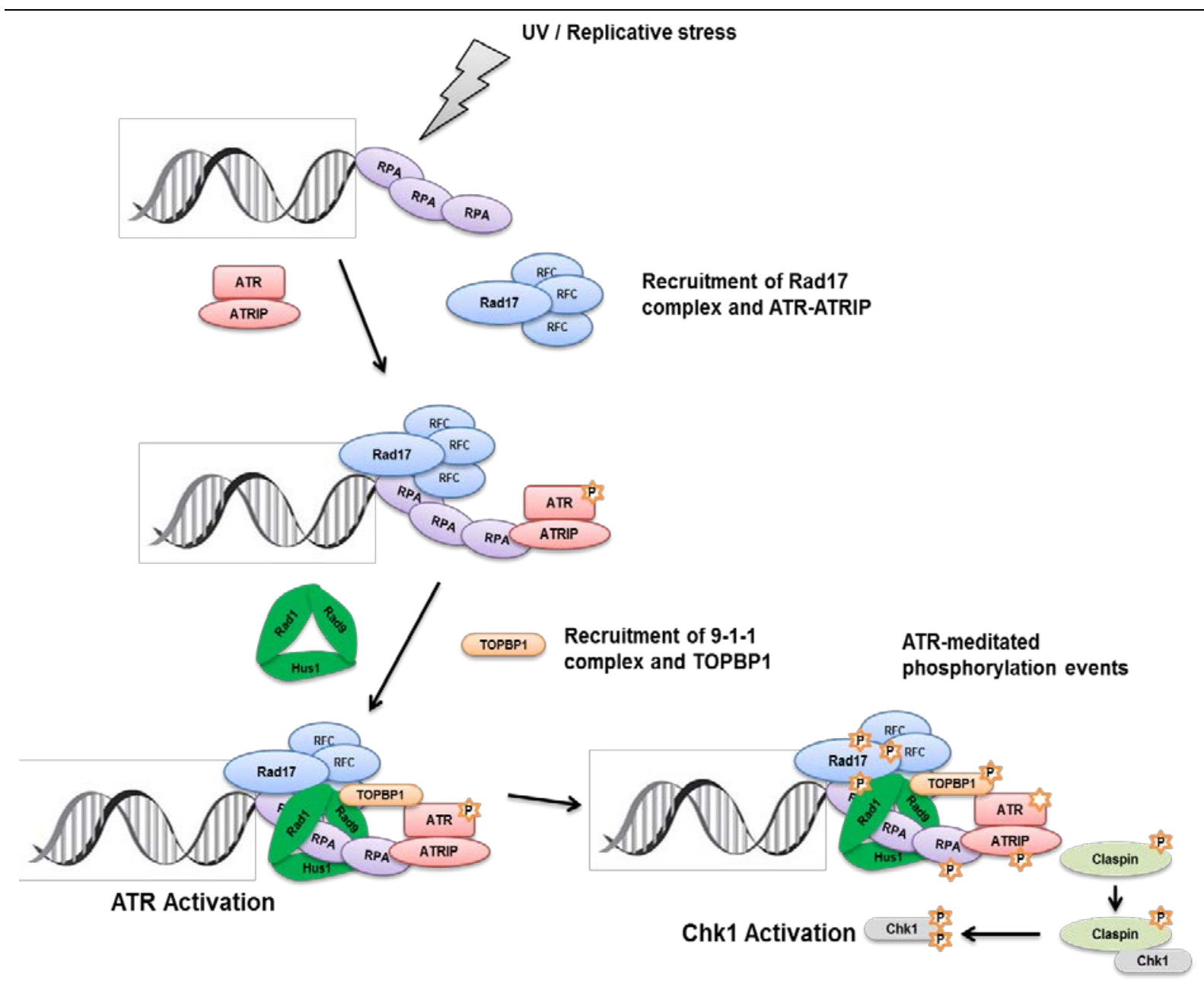

Figure II-II Activation of ATR-Chk1 pathway.

Upon induction of single-strand DNA breaks, a plethora of proteins are recruited to the site of damage and initiates the activation of ATR. Activated ATR, then phosphorylates downstream effector kinase, Chk1 which mediates cell cycle arrest, DNA repair and/or apoptosis. Adapted from (Smits et al. 2010, S. Liu et al. 2011)

\section{II.3.2.2 ATM-Chk2 pathway}

Stalled replication forks can also give rise to DSB either due to replication fork collapse or during processing of the exposed single stranded DNA (Sorensen and Syljuasen 2012). ATR promotes replication fork stabilization at stalled replication forks by controlling replisome-fork association and DNA polymerase stabilization (Cobb et al. 2003, Lucca et al. 2004, (Lopes et al. 2001). If the stalled forks are not stabilized, or if they persist for extended periods of time, they collapse and result in DSB. The situation can be remedied by homologous recombination; further, endonucleases such as Mus81/Eme1 can resolve the transient DNA structures (Sogo, Lopes, and Foiani 2002, Zhou and Elledge 2000).

Following DNA damage, nuclear ATM dimers dissociate into active monomers on autophosphorylation of Ser1981 and localize to sites of DNA damage (Bakkenist and Kastan 2003) via interactions with MRE11/NBS1/RAD50 (MRN) complex (Lee and Paull 
2005). As a central kinase in triggering cellular responses, ATM can phosphorylate several substrates including the variant histone, $\mathrm{H} 2 \mathrm{AX}$, to form the DNA damageassociated histone marker, yH2AX (Fernandez-Capetillo et al. 2004). Additionally, it also phosphorylates the downstream effector kinase Chk2 at Thr68 (Lukas et al. 2003), NBS1 of MRN complex (Bolderson et al. 2004), the cohesin SMC1 (Kitagawa et al. 2004), transcription factor p53 (Lavin and Kozlov 2007), the 'master regulator' of recognition and repair process MDC1, and other repair factors BRCA1 and p53BP1 (Lavin 2008). Once activated, Chk2 acts on multiple substrates involved in cell cycle progression (Cdc25 (Blasina et al. 1999)); apoptosis (p53 (Nabil H. Chehab et al. 2000)) and gene transcription (transcription factors such as E2F1 (Stevens, Smith, and La Thangue 2003)).

\section{Refer to Figure II-III}

ATR is predominantly required for activation of DNA damage checkpoints in response to replication stress, while ATM is the primary mediator for the response to DSB (Yosef Shiloh 2003). Interestingly, evidence from two independent groups suggests activation of ATR by ATM in response to ionizing radiation (IR)-induced DSB (Jazayeri et al. 2006, Myers and Cortez 2006). Moreover, it has been shown that ATM becomes autophosphorylated on its activation site, Ser1981, co-localizes at the sites of replication forks induced by nucleoside analogs, and is required for survival upon induction of DSB in response to IR (Karnitz et al. 2005, Brett Ewald, Sampath, and Plunkett 2007). These findings throw light on the convergence of the two pathways in response to DNA damage.

\section{II.3.2.3 Phosphorylated H2AX (or YH2AX)}

$\mathrm{H} 2 \mathrm{AX}$ belongs to the $\mathrm{H} 2 \mathrm{~A}$ family of histone proteins, one of the five families of histone that package and organize eukaryotic DNA into chromatin. Each nucleosome contains two $\mathrm{H} 2 \mathrm{~A}$ molecules, of which $\sim 10 \%$ are $\mathrm{H} 2 \mathrm{AX}$ in normal human fibroblasts; this translates into an H2AX molecule on every fifth nucleosome on average. However, the percentage of $\mathrm{H} 2 \mathrm{AX}$ can vary from as low as $2 \%$ (in lymphocytes and Hela cells) to as high as $20 \%$ (in SF268, a human glioma cell line) (E P Rogakou et al. 1998). Phosphorylation of H2AX at Ser139 had initially been associated with DSB formation (Emmy P. Rogakou et al. 1998). Apart from ATR and ATM, DNA-PK mediates phosphorylation of H2AX in cells under hypertonic conditions and during apoptotic DNA fragmentation (Reitsema et al. 2005, Mukherjee et al. 2006). Initially, H2AX molecules in a small region near the DSB site are phosphorylated, which is followed by molecules at increasing distances from the break site that can include millions of base pairs (E P Rogakou et al. 1999). Many DNA repair and/or checkpoint proteins accumulate on the growing $\mathrm{yH} 2 \mathrm{AX}$ focus, which may serve to open up the chromatin structure (Kruhlak et al. 2006, Niels Mailand et al. 2007) and form a platform for the accumulation of DNA damage response and repair factors 
(Paull et al. 2000). Once the damaged DNA is repaired, yH2AX foci disappear, probably due to dephosphorylation by phosphatases PP2A and PP4C (Chowdhury et al. 2005, Chowdhury et al. 2008) or removal of $\mathrm{yH} 2 \mathrm{AX}$ from chromatin by histone exchange (Downs et al. 2004)

\section{II.3.3 Cyclin-dependent kinases in the DNA damage response}

In response to DNA damage, Cdks activity is negatively regulated. Apart from regulation by Chk1 and Wee1, various other regulators are present which compete with cyclins in binding to specific Cdks, thereby inhibiting their activity. These regulators have been termed as Cdk inhibitors or CKIs and are classified into two families based on their specificity of interaction with Cdks and sequence homology. One of the families is INK4 which consists of proteins having ankyrin-like repeats. Members of this family are p15 and p16 and they bind to Cdk4/6. Other family is Cip/Kip which includes p21 and p27 and they inhibit Cdk2 (M. H. Lee and Yang 2001). Cdk2 has been proposed to activate Mus81Eme1 endonuclease by its phosphorylation. Moreover, upon depletion of Wee1, hyperactivated Cdk2 can cause increase in Mus81 activity which then triggers DNA damage response (Dominguez-Kelly et al. 2011).

\section{II.3.4 Checkpoint kinases in cell cycle regulation following DNA damage}

Three checkpoints operate during the cell cycle, namely G1-, intra S- and G2/M- phase checkpoints. These checkpoints get activated in response to DNA damage and function to halt the cell cycle progression and signal downstream to repair factors to maintain the integrity of genome as summarized in Figure II-III.

\section{II.3.4.1 The G1 checkpoint}

Regulation of G1 checkpoint has been proposed by a two-wave model wherein a p53 independent, rapid and transient initial response is followed by a delayed, yet sustained, p53-dependent response (Bartek and Lukas 2001). Early response after exposure to IR or UV is mediated by Cdc25A phosphatase degradation, initiated by ATM/ATR pathway activation in response to DNA damage. UV and IR elicit the phosphorylation of Cdc25A at several amino-terminal serine residues by Chk1, downstream of ATR ( $N$ Mailand et al. 2000) and Chk2, activated by ATM (Falck et al. 2001). Phosphorylation of Cdc25A triggers its proteasomal degradation; as a consequence Cdc25A is no longer available to promote the activity of cyclin $E(A) / c d k 2$ by removing the inhibitory phosphates at Thr14 and Tyr15 on Cdk2 (Tse, Carvajal, and Schwartz 2007). The outcome of this cascade is inhibition of Cdk2-dependent loading of Cdc45, an initiator of DNA replication, onto DNA pre-replication complexes (Costanzo et al. 2000). 
A delayed response to sustain $\mathrm{G} 1$ arrest has been ascribed to p53, a tumor suppressor protein that functions as a transcription factor. Upon induction of stress stimuli, p53 is post-transcriptionally modified and stabilized; moreover, its sequence-specific DNA binding is activated (Bert Vogelstein, Lane, and Levine 2000). Once activated, p53 can stimulate the transcription of its target genes; one of them is p21, which inhibits Cdks and therefore, blocks cell cycle progression. However, for the activation of p53, ATM/ATR and Chk2/Chk1 are required, wherein ATM (and also likely ATR) phosphorylates Mdm2 (at Ser395) which deregulates the nuclear transport of p53. It also phosphorylates and activates p53 at Ser15 and some other residues. (Maya et al. 2001, Y. Zhang and Xiong 2001, Kastan and Lim 2000, Y Shiloh 2001, Ryan, Phillips, and Vousden 2001). Phosphorylation of p53 by Chk2/Chk1 at Ser20 helps stabilize p53 by uncoupling it from Mdm2 Ubiquitin ligase ( $\mathrm{N} \mathrm{H}$ Chehab et al. 2000, Hirao et al. 2000, S. Y. Shieh et al. 2000). Thus, two waves of G1 checkpoint are activated simultaneously but their effect on Cdk activity and consequently on G1 blockage varies in time, due to dependence of p53 pathway on transcription and protein synthesis (Bartek and Lukas 2001).

\section{II.3.4.2 The intra-S checkpoint}

The ATR and ATM pathways that operate during $\mathrm{G1}$ are also active during S-phase.In parallel to activation of Chk2, ATM also phosphorylates SMC1 with the aid of BRCA1, FANCD2 and NBS1 that play an active role in repair of DSB or recovery of collapsed replication forks (S.-T. Kim, Xu, and Kastan 2002, Yazdi et al. 2002).

\section{II.3.4.3 The G2/M checkpoint}

The G2/M checkpoint prevents cells from undergoing mitosis in the presence of DNA damage. This checkpoint also employs the ATR-Chk1 or ATM-Chk2 pathways, depending on the type of damage. In both cases, checkpoint kinases inhibit entry into mitosis by down-regulating Cdc25 and up-regulating Wee1, which together control Cdc2/CyclinB activity (Furnari, Rhind, and Russell 1997, Sanchez et al. 1997). Upon phosphorylation, the cdc25 phosphatase binds to 14-3-3 proteins, becomes sequestered in the cytoplasm and is degraded by the ubiquitin-proteasome pathway (C.-Y. Peng et al. 1997). It then leads to accumulation of Tyr15 phosphorylated Cdc2, a substrate for Wee1, that subsequently leads to mitotic arrest. Moreover, MAP kinases p38y (X. Wang et al. 2000) and p38a (Bulavin et al. 2001) have been implicated in G2/M checkpoint response to IR and UV, respectively. 


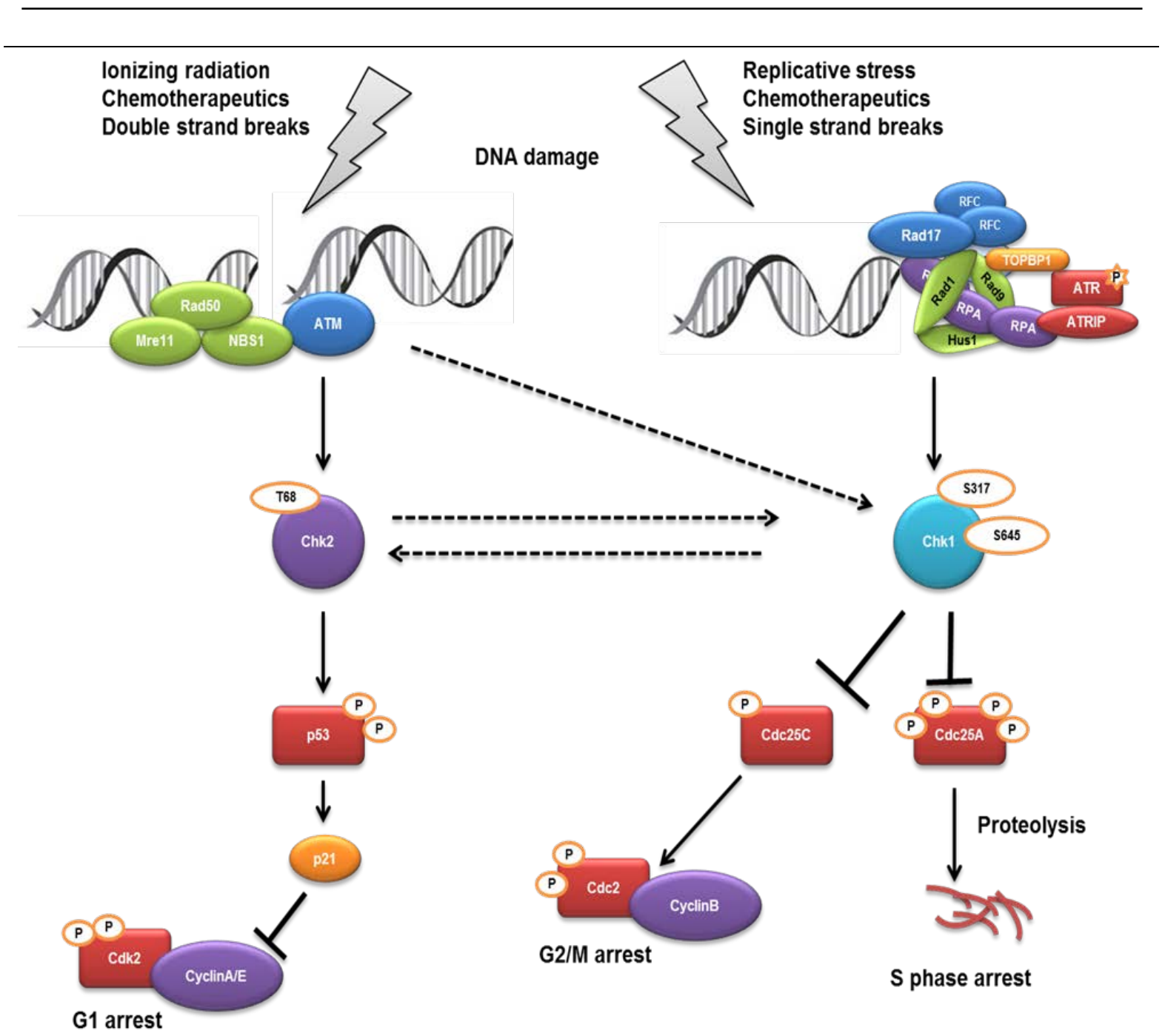

Figure II-III Checkpoint signaling in response to DNA damage.

In response to DNA damage, checkpoint kinases (ATR/ATM) get activated and initiate phosphorylation events to cause cell cycle arrest. Adapted from (Ashwell and Zabludoff 2008)

\section{II.4 Gemcitabine}

Gemcitabine (2', 2'-difluorodeoxycytidine, dFdC) is a deoxycytidine analog having geminal fluorine atoms in the 2'-position of the sugar moiety (as depicted in Figure II-IV). This drug was found to be active in a broad spectrum of solid tumors, as a single agent in the treatment of pancreatic cancer (Burris et. al 1997), in combination chemotherapy of breast cancer (Albain et al. 2008), bladder cancer (von der Maase et al. 2000) and nonsmall cell lung cancer (Sandler et al. 2000). 


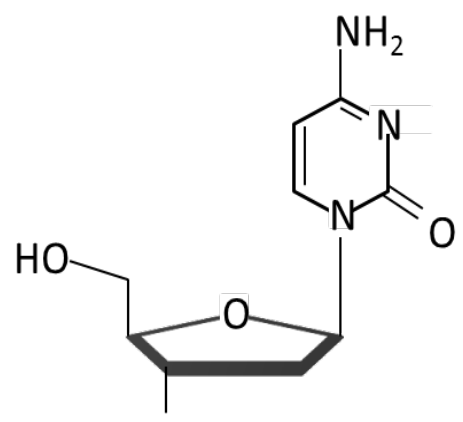

$\mathrm{OH}$

2'-Deoxycytidine

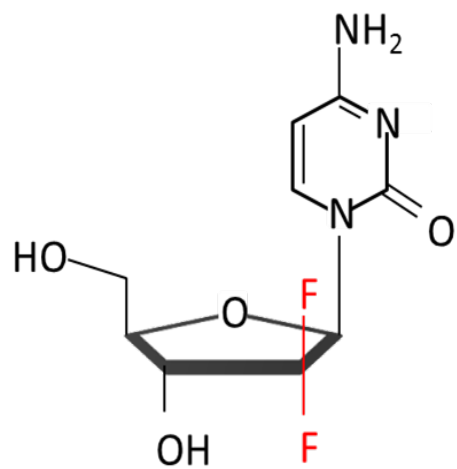

Gemcitabine

Figure II-IV Structures of deoxycytidine and gemcitabine.

Gemcitabine contains geminal fluorine atoms in the 2'-position of the sugar moiety. Adapted from (B Ewald, Sampath, and Plunkett 2008)

Gemcitabine is a prodrug which requires cellular uptake and intracellular phosphorylation. Inside the cell, it is phosphorylated to the active metabolites- gemcitabine di- and triphosphate (dFdCDP and dFdCTP, respectively) (Heinemann et al. 1988). dFdCTP competes with dCTP for incorporation into the DNA (Huang et al. 1991); after incorporation of only one additional nucleotide by DNA polymerase into the DNA chain, it leads to termination of chain elongation. The nonterminal position of dFdCTP in the DNA chain prevents detection and repair by DNA repair enzymes (so-called masked chain termination) (Plunkett et al. 1995).These molecular events are critical for gemcitabineinduced apoptosis.

Efficient phosphorylation and relatively slow elimination ensures high build-up of dFdCTP and $\mathrm{dFdCDP}$ in cells. $\mathrm{dFdCDP}$ is a potent inhibitor of ribonucleotide reductase. Ribonucleotide reductase ( $R R$ ) is the enzyme that mediates conversion of ribonucleotides to deoxyribonucleotides, which is the rate-limiting step in the DNA synthesis. Inhibition of RR impairs DNA synthesis and consequently affects cell proliferation, therefore, it is considered to be an important target for anticancer agents. dFdCDP is falsely recognized by RR as a natural substrate and leads to formation of abnormal products and subsequently to loss of RR catalytic activity. Inhibition of RR causes reduction of deoxynucleotide pools (Baker et al. 1991, van der Donk et al. 1998, Shao et al. 2006, J. Wang, Lohman, and Stubbe 2007). It is also possible that a change in the dFdCTP:dCTP ratio causes enhanced gemcitabine incorporation and further DNA synthesis inhibition, an action known as self-potentiation (Heinemann et al. 1990). Other reported activities of gemcitabine metabolites include the inhibition of cytidine triphosphate synthetase (CTP 
synthetase) (Heinemann et al. 1995) and deoxycytidylate deaminase (dCMP deaminase) by dFdCTP (Heinemann et al. 1992). It has recently been shown that gemcitabine can impede topoisomerase I, suggesting that induction of topoisomerase I-mediated DNA break formation can also contribute to the cytotoxicity of this drug (Pourquier et al. 2002).

\section{II.4.1 DNA damage response generated by gemcitabine}

Gemcitabine exerts its major cytotoxic effect through replication fork stalling, leading to activation of the ATR-Chk1 pathway that maintains genomic stability during replication stress. As discussed in section 1.3.2.1, ATR is an essential replication checkpoint protein which gets activated upon replicative stress and further activates its downstream effector, Chk1. This activates the S-phase checkpoint which blocks cell cycle progression, downregulates origin firing and stabilizes replication forks (Paulsen and Cimprich 2007).

\section{II.4.2 Resistance towards gemcitabine in pancreatic cancer}

According to recent statistics, pancreatic cancer is the eighth leading cause of cancerrelated deaths in the world (Ferlay et al. 2010). Currently, gemcitabine is the leading therapeutic for pancreatic cancer as it improves the survival of patients; however the overall tumor response rate is only 5.4\%, median survival duration of 5.65 months (Burris et. al 1997) and the median progression-free survival is 3.5 months (Moore et al. 2003). In many cases, pancreatic cancer develops resistance to the gemcitabine necessitating further studies into this aspect of cancer.

Many genetic and/or epigenetic alterations have been found to be associated with gemcitabine resistance. These include gene products involved in-

- Transport and metabolism of gemcitabine: Nucleoside transporter-1 (hENT1) is an important element involved in uptake of gemcitabine and its alteration provides gemcitabine resistance to pancreatic tumors (Giovannetti et al. 2006): Metabolic gene products: deoxycytidine kinase and ribonucleoside reductases M1 and M2 have also been related to gemcitabine resistance (Nakano et al. 2007).

- Cell survival or apoptosis: Aberrant expression of S100 can increase resistance partly by modulating hypoxia-induced proapoptotic gene, BNIP3 (Erkan et al. 2005, Mahon et al. 2007).

- Other pathways or proteins implicated in gemcitabine resistance include; phosphatidylinositol 3-kinase/Akt survival pathway ( $\mathrm{Ng}$ et al. 2000, $\mathrm{Ng}$ et al. 2001, Bondar et al. 2002, Asano et al. 2004), activation of the non-receptor protein tyrosine kinases - focal adhesion kinase (M S Duxbury et al. 2004) and c-Src (Mark S. Duxbury et al. 2004). 
Apart from these factors, a plethora of regulators have also been found to decrease gemcitabine sensitivity of pancreatic cancer (Voutsadakis 2011). Consequently, if multiple individual genes contribute to resistance, it would require patient-specific, tailored treatment regimens using specific sensitizers based on the characterization of resistance mechanisms for individual tumors. Another strategy could be to define a 'signature' resistant profile and target it to restore sensitivity, although patient-specific weightage of the variables would need to be taken into account in this case (M. P. Kim and Gallick 2008).

\section{II.5 Targeting cell cycle checkpoint kinases in combination with gemcitabine}

Cancer cells can evade the normal physiological signals for growth and survival by deregulation of kinases. This being one of the major mechanisms for the cancer cell proliferation and survival, has attracted many researchers to design small molecules that target and inhibit kinases (J. Zhang, Yang, and Gray 2009). Checkpoint kinases have emerged as therapeutically important targets as their inhibition could selectively sensitize cancer cells to DNA-damaging agents, thus potentiating the anti-tumor activity and widening the therapeutic margin of these agents. In majority of cancer cells, G1 checkpoint is impaired; as a consequence, these cells rely on S- and G2/M-phase checkpoints for DNA repair and survival (Ashwell and Zabludoff 2008). The known players involved in S- and G2/M- phase checkpoints are ATR, Chk1 and Wee1. Thus, combining inhibitors of these kinases with gemcitabine can sensitize different tumor cells; which includes pancreatic, colon and breast tumors (Prevo et al. 2012a), Zabludoff et al. 2008, (Rajeshkumar, Oliveira, et al. 2011). Since gemcitabine leads to replicative stress in the cells and activates the S-phase checkpoint to counteract the damage to DNA, inhibitors of checkpoint kinases can allow the cells to move through the S-phase even when DNA is not repaired, thus pushing the cells towards cell death.

\section{II.6 The kinase Wee1 - a regulator of Cdks}

Wee1 is a nuclear protein and a serine/threonine and tyrosine kinase which negatively regulates the activity of Cdks by phosphorylating them at Tyr15 (Parker and PiwnicaWorms 1992) and hence, controls the cell cycle progression. The protein levels and activity of Wee1 are tightly regulated during cell cycle; they peak during S- and G2phases of the cell cycle (N Watanabe, Broome, and Hunter 1995). During G2/M transition, 
hyperphosphorylation and degradation of Wee1 has been observed ( $N$ Watanabe, Broome, and Hunter 1995). At the onset of mitosis, Wee1 is phosphorylated by cdc2 (or cdk1) and Polo-like kinase1 (Plk1) at Ser123 and Ser53; these phosphorylation sites are recognized by the SCF $\beta-\operatorname{TrCP} 1 / 2$, an E3 ubiquitin ligase, for Wee1 ubiquitination (Nobumoto Watanabe et al. 2004).

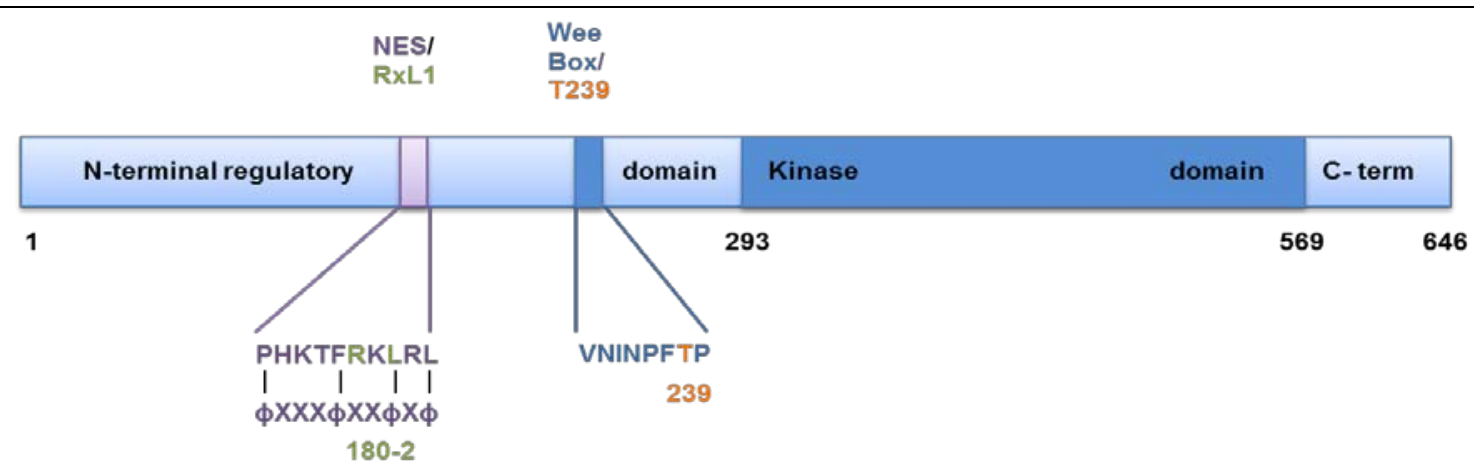

Figure II-V Primary structure of the human Wee1 protein.

The NRD, kinase domain, and short carboxy-terminal domain are marked, with border amino acid residues numbered (below). The T239 Cdk phosphorylation site, an inhibitory modification, resides within the Wee box, a positive regulatory element. Adapted from (Enders 2010)

Several other kinases have also been reported to phosphorylate and activate Wee1. In Xenopus, it has been shown that 14-3-3 binds to Wee1; this requires prior phosphorylation at Ser549 near the C-terminus of Wee1; Chk1 was shown to phosphorylate Ser549 (Ser642 in human Wee1). In contrast, in humans, it has been suggested that other kinases may play this role (J. Lee, Kumagai, and Dunphy 2001, Rothblum-Oviatt, Ryan, and Piwnica-Worms 2001). Wee1 is also positively regulated by autophosphorylation- three sites on the Xenopus Wee1 (Tyr90, Tyr103 and Tyr110) and two sites on the human Wee1 (Tyr295 and Tyr362) have been identified as autophosphorylation sites (Murakami, Copeland, and Vande Woude 1999, Katayama, Fujita, and Tsuruo 2005). Moreover, it has been reported that the activity of Wee1 can also be stimulated by the Cdk-interacting protein Cables (C. L. Wu et al. 2001).

DNA damage (e.g., radiation or UV irradiation) activates Chk1, which promotes G2/M arrest through phosphorylation of Cdc25C and Wee1. Cdc25C is a phosphatase that dephosphorylates Tyr15 of Cdc2 and thus, activates it (A Kumagai and Dunphy 1991). Cdc25C is phosphorylated on Ser216 by Chk1 and the phosphorylated form of Cdc25C binds to 14-3-3 and eliminates its functions through translocation to the cytoplasm (C. Y. Peng et al. 1997). Moreover, Chk1 may also phosphorylate Wee1 at Ser642. Although it is not yet reported in humans, phosphorylated Ser642 increases the stability of Wee1 in the nucleus by binding to $14-3-3 \beta$ or $-\sigma$ in Xenopus and through yeast two-hybrid 
screening (J. Lee, Kumagai, and Dunphy 2001, Y. Wang et al. 2000). As a result, Cdc2 is continuously phosphorylated at Tyr15, and the cell cycle arrests at the G2/M transition. This way, the balance between Cdc25 and Wee1 is tightly controlled through the cell cycle so that cells undergo G2/M transition without damaged DNA.

\section{II.6.1.1 Inhibition of Wee1 and genomic instability in cancer}

Wee1 depletion has been found to induce replicative stress. Inhibition of Wee1 kinase elevates Cdk activity that rapidly increases initiation of replication. Firing of replication origins is increased, followed by shortage of nucleotides and reduction in replication fork speed, and subsequent generation of DNA double-strand breakage mediated by SLX4/MUS81. Interestingly, depletion of a key factor for replication initiation, known as CDT1, leads to normalized fork speed and suppressed DNA DSB formation. Furthermore, addition of nucleosides counteracts the effects of unscheduled Cdk activity on fork speed and DNA DSB formation (Beck et al. 2012).

It has been shown that cells arrested in S-phase enter directly into mitosis without completely replicating DNA when Wee1 is inhibited in these cells (also known as premature mitosis). This results in highly unusual mitoses identified by scattered chromosomes and disordered spindle fibers, which eventually leads to exit of cells from mitosis with many micronuclei formation and apoptosis (Aarts et al. 2012). When cells enter into mitosis prematurely with unrepaired lethal DNA damage, it results in mitotic catastrophe. Thus, Wee1 inhibition pushes the cells to mitotic catastrophe when combined with DNA-damaging agents, especially in p53 deficient cancer cell (Hamer et al. 2011).

\section{II.6.1.2 Mitotic catastrophe versus apoptosis}

Mitotic catastrophe (MC) is the process resulting from abnormal or premature mitosis and is characterized by the formation of multinucleated cells and leads to cell death. Cell death could occur by apoptosis or necrosis either during or after dysregulated mitosis. When cells enter into mitosis, histone $3(\mathrm{H} 3)$ gets phosphorylated at Ser10 and plays a part in complex signaling network and besides serve as mitotic marker (Hans and Dimitrov 2001, Tsuta et al. 2011). Alternately, mitotic cells can be stained with MPM-2 anitbody (mitotic phosphoprotein monoclonal antibody 2), this antibody recognize a subset of proteins having mitotically phosphorylated S/TP motifs (peptides containing LTPLK and FTPLQ domains) (C. F. Wu et al. 2010). Cells undergoing premature mitosis stain positive for the above mentioned mitotic markers and can be identified using flow cytometry; this gives a quantifiable indication of cells going through MC. MC is considered to prevent genomic instability in the cells and its disruption promotes tumorigenesis and cancer progression (Vitale et al. 2011) 
Apoptosis is the process of programmed cell death and is one of the modes of removing damaged cells and thus, help prevent tumorigenesis. Cells undergoing apoptosis are characterized by chromatin condensation, extensive plasma membrane blebbing and nuclear fragmentation. Apoptotic cells are afterwards phagocytosed by macrophages, parenchymal cells, or neoplastic cells and degraded within phagolysosomes (Elmore 2007). Several apoptotic pathways in cells responsive to apoptotic stimuli have been suggested, such as the death receptor-mediated pathway, the mitochondrial apoptotic pathway, and the endoplasmic reticulum pathway. Although initial induction mechanisms are different for each pathway, they converge at a common final phase of apoptosis, consisting of the activation of the executioner caspases and cleaving of substrates critical for cell survival. It has been reported that in response to chemotherapeutic treatment, mitochondrial pathway is largely activated wherein permeability of mitochondrial membrane is increased resulting in release of apoptotic components such as cytochrome c, apoptosis-inducing factor (AIF) and endonuclease G. These proteins eventually activate caspase which leads to cell death. However, mammalian cells can undergo caspase-independent apoptosis under certain circumstances. Caspase-independent apoptosis is mediated by the disruption of the mitochondrial membrane potential and the translocation of AIF and endonuclease $G$ to the nucleus where they induce chromatin condensation and/or large-scale DNA fragmentation (T.-J. Lee et al. 2006).

\section{II.6.1.3 Integration of mitotic catastrophe and apoptosis}

In some instances, $\mathrm{MC}$ is accompanied by the hallmarks of apoptosis suggesting the interaction of checkpoints or sensors of mitotic failure and apoptotic machinery (Castedo et al. 2006). During metaphase-arrest, Cdk1 can phosphorylate anti-apoptotic proteins, such as Bcl-xl (a member of Bcl-2 protein family) (Terrano, Upreti, and Chambers 2010) and survivin (Barrett, Osborne, and Wheatley 2009) and inhibit their activity, thereby facilitating cell death. In addition to metaphase arrest-induced apoptosis, tetraploid cells that are generated through catastrophic mitosis followed by mitotic slippage also undergo apoptosis. It is evidenced by an immediate induction of p21 after mitotic slippage, which is an indicator of a p53-dependent checkpoint response in G1-phase (Vogel et al. 2004). Apoptosis, however, is not always required for MC lethality, as some multinucleated cells can undergo slow death in a necrosis-like manner (Eom et al. 2005).

\section{II.7 Polo-like kinase1 and its role in ATR-Chk1 pathway}

Polo-like kinases (Plks) are emerging as key regulators of essential cell cycle events. Plk family in mammals is comprised of four members- Plk1, 2, 3 and 4. Proteins belonging to 
this family contain an N-terminal Ser/Thr kinase catalytic domain and a C-terminal region containing two conserved Polo-box regions. Plk1 localizes to the cytoplasm and centrosome during interphase and concentrates to kinetochores and the cytokinesis bridge during cell division. Thus, it plays a major role in centrosome maturation, mitotic entry, and cytokinesis (Archambault and Glover 2009). The Polo-box domain (PBD) of Plk1 plays a unique role in subcellular localization and mediates protein interactions (Cheng et al. 2003, Elia et al. 2003). It is a phospho-peptide binding domain that binds to the proteins 'primed' or phosphorylated by kinases, thereby facilitating localization of Plk1. Kinases known to prime Plk1 substrates include Cdk1, that drives the cells into mitosis (K. S. Lee et al. 2008). Plk1 activity is also regulated by Aurora A kinase through an auxiliary protein, Bora (Seki et al. 2008). As shown in Figure II-VI, binding of Bora to Plk1 facilitates the phosphorylation of Plk1 at Thr210 by Aurora A causing the activation of Plk1.

Plk1 has been identified as a novel modulator of DNA damage checkpoints, where it maintains genomic stability during DNA replication (Takaki et al. 2008). It facilitates recovery from DNA damage checkpoint-mediated arrest at G2/M phase following successful DNA damage repair (Niels Mailand et al. 2006, van Vugt, Brás, and Medema 2004). After DNA damage, phosphorylation of Thr210 of Plk1 is inhibited that targets Plk1 for degradation by the anaphase-promoting complex/cyclosome (APC/C) bound to its activator Cdh1; this prevents entry of the cells into mitosis. It has been found that successful resumption of cell cycle progression at G2/M and mitotic entry relies on the activation of Plk1 by Aurora A/Bora-mediated phosphorylation of Thr210 within the activation loop of Plk1 (Macůrek et al. 2008, Seki et al. 2008). Subsequently, Plk1 induces degradation of Wee1, a kinase that inhibits Cdk1 (van Vugt, Brás, and Medema 2004), and Claspin, an adaptor protein that is required to sustain Chk1 and checkpoint activity (Niels Mailand et al. 2006a), Mamely et al. 2006). Phosphorylation of a sequence in Claspin's amino-terminus, called phosphodegron, by Plk1 marks Claspin for degradation by the SCF $\beta-\operatorname{TrCP} 1 / 2$ ubiquitin ligase. Elimination of Wee1 and Claspin contributes to Cdk1 activation and leads to mitotic entry.

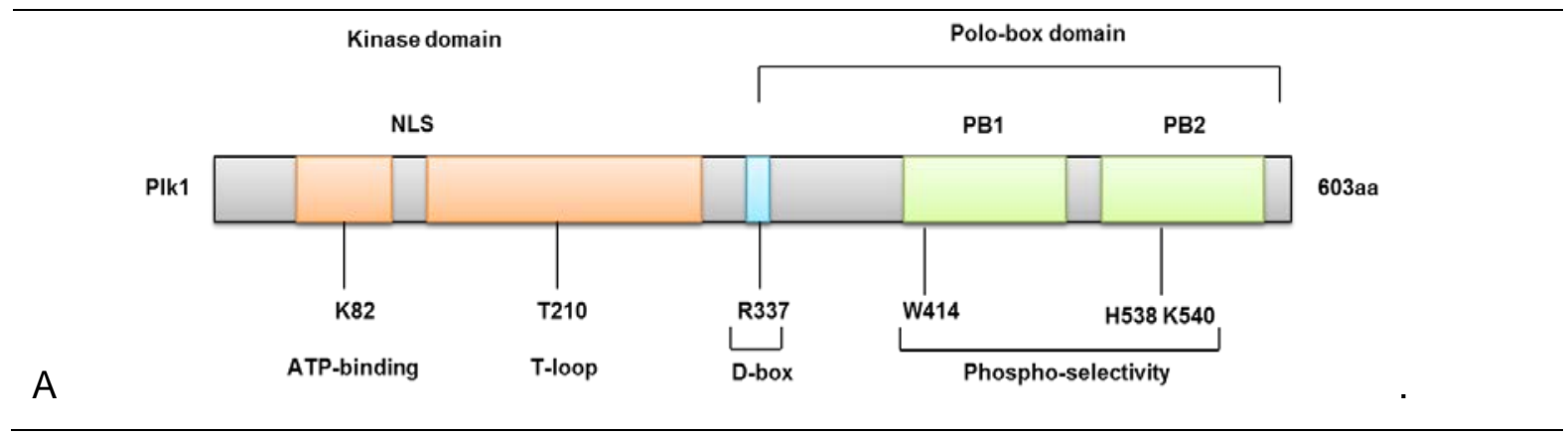




\section{Bora}

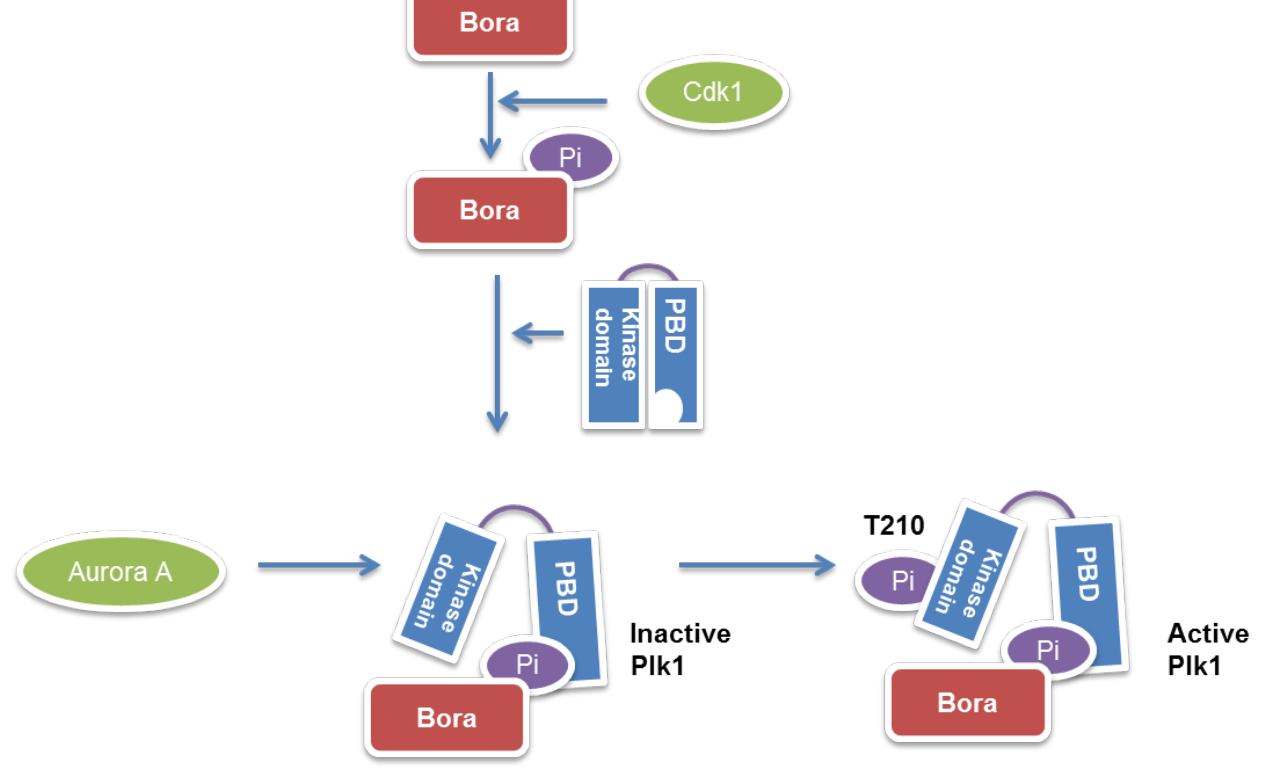

B.

Figure II-VI A. Structure of human PIk1.

The positions of the kinase domains (red) and polo-boxes 1 and 2 (blue) are also depicted. The PLK1 sequences that mediate its nuclear localization (NLS, which is indicated in green) and its destruction at the end of mitosis (D-box, which is indicated in yellow) are also shown. Residues that are crucial for ATP-binding and enzymatic activation (T-loop) within the kinase domains, and phospho-selectivity within the polo-boxes are indicated. Adapted from (Strebhardt and Ullrich 2006)

\section{B. Priming of Bora by Cdk1 for Plk1 activation by Aurora A.}

Cdk1 phosphorylates Bora, which is recognized by Plk1. Plk1 undergoes a conformational change and Thr210 is exposed to be phosphorylated by Aurora A. This phosphorylation, in turn, activates Plk1. Adapted from (K. S. Lee et al. 2008)

Interestingly, it has been shown that DNA damage checkpoints can regulate signaling pathways upstream of Plk1. Plk1 is phosphorylated at Ser137 and Thr210 during mitosis, and phosphorylation at these sites is prevented during DNA damage through ATR/ATMdependent signaling pathways in asynchronous cells (Tsvetkov and Stern 2005).

\section{II.8 Nutlin- 3, as a protector of p53-proficient cells against nucleoside analogues}

p53 is a tumor suppressor protein that prevents the propagation of DNA damage which may lead to malignant cell transformation (Levine 1997, B Vogelstein, Lane, and Levine 2000). As discussed in section 1.4.3, p53 levels are tightly controlled by its negative regulator, Mdm2 that binds to p53 and modulates its transcriptional activity and stability (Oliner et al. 1993, Freedman, Wu, and Levine 1999). Upon DNA damage, Mdm2 levels fall and p53 levels increase, which induces $\mathrm{G} 1$ arrest that is mediated by its immediate downstream target gene product p21/Waf1/Cip1 (El-Deiry et al. 1993). 
Nutlin-3 is a small molecule inhibitor of Mdm2-p53 interaction, thereby, causing nongenotoxic accumulation of p53 which results in cell cycle arrest and/or apoptotic response (Miyachi et al. 2009). Nutlin-3 has been found to selectively activate wild-type p53 and not mutant p53. This differential activity of Nutlin-3 can be utilized to protect the normal proliferating cells (having wild-type p53) from cytotoxicity of mitotic inhibitors or S-phase specific chemotherapeutics used to treat tumor cells (either p53-deficient or having mutant p53) (Carvajal et al. 2005). Due to the tumor suppressive role of p53, it is mutated in about $50 \%$ of human tumors rendering them insensitive to p53- activating agents ( $B$ Vogelstein, Lane, and Levine 2000, Hollstein et al. 1991). This provides the opportunity to develop an improved strategy for protection of normal proliferating tissues without affecting the sensitivity of tumors with mutant p53 to certain chemotherapeutics. It has been shown that pretreatment with nutlin before chemotherapy with antimitotic agents (paclitaxel) and S-phase abrogators (gemcitabine) can offer partial protection to normal proliferating tissues by causing cell cycle arrest in G1 and G2-M phase (Carvajal et al. 2005, Kranz and Dobbelstein 2006).

\section{II.9 Scope of the thesis}

Combining checkpoint inhibitors with gemcitabine provides a promising way of sensitizing tumors. However, there are no studies which compare quantitatively the efficacy of the inhibition of different checkpoint kinases with gemcitabine. In this project, we have tried to address this question and the questions arising from it.

Inhibition of Wee1 checkpoint kinase sensitizes tumor cells towards the chemotherapeutic drug, gemcitabine. Various studies have been performed to determine how Wee1 inhibition in combination with gemcitabine could lead to enhanced cytotoxicity. Here, we asked whether Wee1 inhibition interferes with the activity of additional checkpoint kinases, thereby enhancing cytotoxicity.

Nutlin can act as protector for normal proliferating cells against gemcitabine while it does not affect the sensitivity of tumor cells with mutant p53 towards chemotherapy. Still, the protective role of Nutlin has not been analyzed for combinations of gemcitabine with checkpoint kinase inhibitors. We therefore asked whether nutlin pretreatment, protects cells against subsequent combination therapy of gemcitabine with Wee1 inhibition. 


\section{MATERIALS}

\section{III.1 Technical devices}

Table III.1-1 Technical Devices

Device

Blotting chamber
Cell counting chamber Neubauer improved
Centrifuge 5415R
Centrifuge 5810R
Centrifuge Megafuge 1.0R

Chemiluminescence imager Chemocam HR 163200

Cytometer Celigo

DNA gel chamber

Electrophoresis system, for SDS-PAGE

FACS machine Guava PCA-96 Base

System

FACS machine FACScanto II

Foil swelding machine Vacupack plus

Freezer $-20^{\circ} \mathrm{C}$

Freezer $-80^{\circ} \mathrm{C}$

Heating Block

Heating Block HLC

Ice-machine $B 100$

Incubator for cell culture Hera Cell 150

Laminar flow cabinet Hera Safe

Liquid nitrogen tank LS 4800

Magnetic stirrer MR Hei-Standard

Magnetic stirrer MR3001

Microscope Axiovert 40C

Microscope, automated Pathway 855

Microscope, confocal Zeiss Confocal LSM

510 meta

Mini Centrifuge MCF-2360

Multichannel Pipette Transferpette S-8

PCR machine for qPCR CFX96, C1000

PCR machine Thermocycler T personal

Personal computer

pH-meter WTW-720

Pipets Eppendorf Research Series 2100

(0.1-2.5 $\mu \mathrm{L} ; 2-20 \mu \mathrm{L} ; 20-200 \mu \mathrm{L} ; 100-1000 \mu \mathrm{L})$

Power supply unit Powerpack P25T

Refrigerator $4^{\circ} \mathrm{C}$

\section{Company}

Biozym, Hessisch Oldendorf, Germany

Brand, Wertheim, Germany

Eppendorf, Hamburg, Germany

Eppendorf

Heraeus, Thermo Scientific, Waltham, MA, United States

Intas Science Imaging Instruments, Göttingen, Germany

Cyntellect, San Diego, CA, United States

Biotech Service Blu, Schauenburg,

Germany

Amersham Biosciences, GE Healthcare, Little Chalfont, United Kingdom

Millipore, Merck, Darmstadt, Germany

Becton Dickinson, Franklin Lakes, NJ, United States

Krups, Groupe SEB, Lyon, France

Liebherr, Bulle, Switzerland

Heraeus, Thermo Scientific

Grant Instruments, Hillsborough, NJ, United

States

HLC Biotech, Ditabis, Pforzheim, Germany

Ziegra, Isernhagen, Germany

Heraeus, Thermo Scientific, Waltham, MA, United States

Heraeus, Thermo Scientific

Taylor-Wharton, Theodore, AL, United

States

Heidolph, Schwabach, Germany

Heidolph

Zeiss, Oberkochen, Germany

Becton Dickinson, Franklin Lakes, NJ, United States

Zeiss

LMS, Tokyo, Japan

BrandTech Scientific, Inc

Bio-Rad Laboratories

Biometra, Göttingen, Germany

Dell, Round Rock, TX, United States

WTW, Weilheim, Germany

Eppendorf

Biometra

Liebherr 
Roller RM5 V-30

Scales Acculab ALC-6100.1

Scales LE623S

Scanner CanoScan 8600F

Shaker PROMAX 2020

Shaker POL YMAX 2040

Shaker VXR Basic Vibrax

Sonication device Bioruptor

Spectrophotometer NanoDrop ND-1000

Thermomixer comfort

Timer

Vacuum pump

Vortex Genie 2

Water bath TW 20
CAT, Staufen, Germany

Sartorius, Göttingen, Germany

Sartorius

Canon, Tokyo, Japan

Heidolph

Heidolph

Ika

Diagenode, Liège, Belgium

PeqLab, Erlangen, Germany

Eppendorf

Oregon Scientific, Portland, OR, United States

IBS Integra Biosciences, Fernwald, Germany

Scientific Industries, Bohemia, NY, United States

Julabo Labortechnik, Seelbach, Germany

\section{III.2 Consumables}

Table III.2-1 Consumables

\section{Product}

96-well plates for microscopy, clear bottom 96-well plates for microscopy, clear bottom 96-well plates for qPCR

Cell culture dishes $(10 \mathrm{~cm}, 15 \mathrm{~cm})$

Cell culture plates (6-well, 12-well)

Cell scraper $(16 \mathrm{~cm}, 25 \mathrm{~cm})$

Cover slips

Cryo tubes Cryoline

Filter tips $(10 \mu \mathrm{L})$

Filter tips $(20 \mu \mathrm{L}, 200 \mu \mathrm{L}, 1,000 \mu \mathrm{L})$

Glass Slides Superfrost

Parafilm

Pipet tips (10 $\mu \mathrm{L}, 20-200 \mu \mathrm{L}, 1,000 \mu \mathrm{L})$

Protran nitrocellulose transfer membrane

PVDF membrane Amersham Hybond-P

Reaction tube $(0.2 \mathrm{~mL})$

Reaction tube $(0.5 \mathrm{~mL}, 1.5 \mathrm{~mL}, 2.0 \mathrm{~mL})$

Reaction tube $(15 \mathrm{~mL}, 50 \mathrm{~mL})$

Sealing foil for 96 -well plate

Sterile filter

Syringe

Syringe canula (different sizes)

Transparent sealing foil for 96 -well plate

Whatman paper

\section{Company}

Becton Dickinson

Corning, Corning, NY, United States

4titude, Wotton, United Kingdom

Greiner, Frickenhausen, Germany

Greiner

Sarstedt

Menzel, Thermo Scientific

Nunc, Thermo Scientific

Starlab, Hamburg, Germany

Sarstedt

Menzel, Thermo Scientific

Brand

Greiner

Whatman, Dassel, Germany

GE Healthcare, Life Sciences

Sarstedt

Eppendorf

Greiner

Thermo Scientific

Millipore, Merck

Henke-Sass, Wolf, Tuttlingen, Germany

B.Braun, Melsungen, Germany

4titude

Whatman 


\section{III.3 Chemicals and reagents}

Table III.3-1 Chemicals and reagents

\section{Substance}

Acetic acid

Agarose

Albumin Fraction $\vee$ (Bovine Serum Albumine, BSA)

Ammonium persulfate (APS)

Ammonium sulfate $\left(\left(\mathrm{NH}_{4}\right)_{2} \mathrm{SO}_{4}\right)$

Ampicillin

Bromophenol blue

Calcium chloride dihydrate $\left(\mathrm{CaCl}_{2} \times 2 \mathrm{H}_{2} \mathrm{O}\right)$

Chloroform

Complete Mini Protease Inhibitor

Dimethyl sulfoxide (DMSO)

Dithiotreitol (DTT)

DNA ladder

deoxynucleotide triphosphates (dNTPs)

Ethanol $99.8 \%$

Ethanol $99.9 \%$ p.a. (EtOH)

Ethidium bromide (EtBr)

Ethylene diamine tetraacetatic acid (EDTA)

Formaldehyde, $37 \%$ solution

Glycerine

Glycine

Glycogen blue

Guava ICF Cleaning Solution

HEPES

Hoechst 33342 (Hoechst)

Hydrogen chloride $(\mathrm{HCl})$

Immersion oil

Isopropanol

Lipofectamine 2000

Magnesium chloride $\left(\mathrm{MgCl}_{2}\right)$ for PCR

Magnesium chloride hexahydrate $\left(\mathrm{MgCl}_{2} \mathrm{X}\right.$

$6 \mathrm{H}_{2} \mathrm{O}$ )

Methanol >99\% (MetOH)

Nailpolish

Nonidet P-40 substitute (NP-40)

Nuclease free water

Ponceau S

Potassium chloride $(\mathrm{KCl})$

Potassium hydrogenphosphate $\left(\mathrm{KH}_{2} \mathrm{PO}_{4}\right)$

Prestained Protein Ladder

Propidium iodide $(\mathrm{PI})$

Protein-G-Sepharose (PGS) 4Fast Flow

RNase inhibitor

Rotiphorese Gel 30

Sodium bicarbonate $\left(\mathrm{NaHCO}_{3}\right)$

Sodium chloride $(\mathrm{NaCl})$

Sodium deoxycholate

\section{Company}

Roth, Karlsruhe, Germany

Roth

Roth

Roth

Roth

AppliChem, Darmstadt, Germany

Sigma-Aldrich

Roth

Roth

Roche, Basel, Schweiz

AppliChem

Sigma-Aldrich

Fermentas, Thermo Scientific

Bio-Budget, Krefeld, Germany

Roth

Merck

Sigma-Aldrich

Roth

Roth

Roth

Roth

Ambion, Life Technologies

Millipore, Merck

Roth

Invitrogen, Life Technologies

Roth

Zeiss

Th. Geyer, Renningen, Germany

Invitrogen, Life Technologies

Fermentas, Thermo Scientific

Roth

Roth

Sigma Aldrich

Ambion, Life Technologies

Roth

Roth

Roth

Fermentas, Thermo Scientific

Sigma-Aldrich

GE healthcare

Fermentas, Thermo Scientific

Roth

Roth

Roth

Applichem 
Sodium dodecyl sulfate (SDS)

Sodium fluoride

Sodium hydrogenphosphate heptahydrate

$\left(\mathrm{Na}_{2} \mathrm{HPO}_{4} \times 7 \mathrm{H}_{2} \mathrm{O}\right)$

Sodium hydroxide $(\mathrm{NaOH})$

Sodium orthovanadate

Sodium pyrophosphate decahydrate

Sucrose

SYBR green

Tetracycline

Tetramethylethylenediamine (TEMED)

Thymidine

Trasylol

Trehalose

Trisamine (Tris)

Triton X-100

Trizol

Tween 20

Vectashield mounting medium

\section{III.4 Buffers and solutions}

Cell lysis buffer

\begin{tabular}{lr}
\hline Urea & $2.5 \mathrm{M}$ \\
RIPA lysis buffer & $100 \%$ \\
$\Rightarrow$ for SDS PAGE, diluted with 6x & \\
Laemmli 1:5 &
\end{tabular}

DNA gel loading buffer, $6 x$

\begin{tabular}{lr}
\hline Sucrose & $40.00 \%$ \\
Glycerin & $10.00 \%$ \\
Bromophenol blue & $0.25 \%$ \\
dissolved in $\mathrm{H}_{2} \mathrm{O}$ &
\end{tabular}

IF blocking solution

\begin{tabular}{lr}
\hline $\begin{array}{l}\text { BSA } \\
\text { dissolved in PBS }\end{array}$ & $3 \%$ \\
& \\
IP buffer & \\
\hline Tris, pH 7.5 & $50 \mathrm{mM}$ \\
$\mathrm{NaCl}$ & $300 \mathrm{mM}$ \\
$\mathrm{NP}-40$ & $1 \%$ \\
$\mathrm{Na}$ deoxycholate & $0.1 \%$ \\
$\mathrm{Na}$ fluoride & $10 \mathrm{mM}$ \\
Na pyrophosphate & $2 \mathrm{mM}$ \\
Protease inhibitors & \\
dissolved in $\mathrm{H}_{2} \mathrm{O}$ &
\end{tabular}

Roth

Roth

Roth

Sigma-Aldrich

Sigma-Aldrich

Applichem

Sigma-Aldrich

Invitrogen, Life Technologies

Sigma-Aldrich

Roth

Sigma-Aldrich

Bayer, Leverkusen, Germany

Sigma-Aldrich

Roth

Applichem

Invitrogen, Life Technologies

Applichem

Vector Laboratories, Burlingame, CA, United States

Laemmli buffer, $6 x$

\begin{tabular}{lr}
\hline Tris pH 6.8 & $0.35 \mathrm{M}$ \\
Glycerin & $30.00 \%$ \\
SDS & $10.00 \%$ \\
Dithiotreitol & $9.30 \%$ \\
Bromophenol blue & $0.02 \%$ \\
dissolved in $\mathrm{H}_{2} \mathrm{O}$ &
\end{tabular}

Phophate buffered saline (PBS), pH 7.5

\begin{tabular}{lr}
\hline $\mathrm{NaCl}$ & $24.00 \mathrm{mM}$ \\
$\mathrm{KCl}$ & $0.27 \mathrm{mM}$ \\
$\mathrm{Na}_{2} \mathrm{HPO}_{4} \times 7 \mathrm{H}_{2} \mathrm{O}$ & $0.81 \mathrm{mM}$ \\
$\mathrm{KH}_{2} \mathrm{PO}_{4}$ & $0.15 \mathrm{mM}$ \\
dissolved in $\mathrm{H}_{2} \mathrm{O}$ &
\end{tabular}

PBS $^{++}$

\begin{tabular}{lr}
\hline $\mathrm{NaCl}$ & $24.00 \mathrm{mM}$ \\
$\mathrm{KCl}$ & $0.27 \mathrm{mM}$ \\
$\mathrm{Na}_{2} \mathrm{HPO}_{4} \times 7 \mathrm{H}_{2} \mathrm{O}$ & $0.81 \mathrm{mM}$ \\
$\mathrm{KH}_{2} \mathrm{PO}_{4}$ & $0.15 \mathrm{mM}$ \\
$\mathrm{CaCl}_{2} \times 2 \mathrm{H}_{2} \mathrm{O}$ & $1.00 \mathrm{mM}$ \\
$\mathrm{MgCl}_{2} \times 6 \mathrm{H}_{2} \mathrm{O}$ & $0.50 \mathrm{mM}$ \\
dissolved in $\mathrm{H}_{2} \mathrm{O}$ & \\
& \\
Ponceau S solution & \\
\hline Ponceau S & $0.5 \%$ \\
Acetic acid & $1.0 \%$ \\
dissolved in $\mathrm{H}_{2} \mathrm{O}$ &
\end{tabular}


qPCR reaction buffer, 10x

$\begin{array}{lr}\text { Tris, } \mathrm{pH} 8,8 & 750 \mathrm{mM} \\ \left(\mathrm{NH}_{4}\right)_{2} \mathrm{SO}_{4} & 200 \mathrm{mM} \\ \text { Tween } 20 & 0.1 \% \\ \text { dissolved in } \mathrm{H}_{2} \mathrm{O} & \end{array}$

qPCR reaction mix, 25x

\begin{tabular}{lr}
\hline $10 x$ qPCR & $1 x$ \\
buffer & \\
SybrGreen & $1: 80,000$ \\
$\mathrm{MgCl}_{2}$ & $3.0 \mathrm{mM}$ \\
Trehalose in $10 \mathrm{mM}$ & $300.0 \mathrm{mM}$ \\
Tris, pH 8,5 & \\
dNTPs & $0.2 \mathrm{mM}$ \\
Triton X-100 & $0.25 \%$ \\
Taq polymerase & $20 \mathrm{U} / \mathrm{mL}$
\end{tabular}

Dissolved in $\mathrm{H}_{2} \mathrm{O}$

RIPA lysis buffer, pH 7.5

\begin{tabular}{lr}
\hline Triton X-100 & $1.0 \%$ \\
$\mathrm{Na}$ deoxycholate & $1.0 \%$ \\
$\mathrm{SDS}$ & $0.1 \%$ \\
$\mathrm{NaCl}$ & $150 \mathrm{mM}$ \\
EDTA & $10 \mathrm{mM}$ \\
Tris, pH 7.5 & $20 \mathrm{mM}$ \\
Trasylol & $50,000 \mathrm{KIU}$ \\
dissolved in $\mathrm{H}_{2} \mathrm{O}$ & \\
& \\
SDS running buffer & \\
\hline Tris & \\
Glycin & $25.0 \mathrm{mM}$ \\
SDS & $86.1 \mathrm{mM}$ \\
dissolved in $\mathrm{H}_{2} \mathrm{O}$ & $3.5 \mathrm{mM}$
\end{tabular}

TAE buffer

\begin{tabular}{lr}
\hline Tris & $40 \mathrm{mM}$ \\
Acetic acid & $20 \mathrm{mM}$ \\
EDTA & $2 \mathrm{mM}$
\end{tabular}

dissolved in $\mathrm{H}_{2} \mathrm{O}$

Tris buffered saline + Tween 20 (TBST), pH 7.6

\begin{tabular}{lr}
\hline Tris & $50 \mathrm{mM}$ \\
$\mathrm{NaCl}$ & $150 \mathrm{mM}$ \\
Tween 20 & $0.1 \%$ \\
dissolved in $\mathrm{H} 2 \mathrm{O}$ &
\end{tabular}

\begin{tabular}{l} 
Western blot blocking solution \\
\hline BSA $5 \%$ \\
dissolved in TBST
\end{tabular}

Western blot buffer, pH 8.3

\begin{tabular}{lr}
\hline Tris & $25 \mathrm{mM}$ \\
Glycin & $192 \mathrm{mM}$ \\
MetOH & $20 \%$ \\
dissolved in $\mathrm{H}_{2} \mathrm{O}$ &
\end{tabular}

\section{III.5 Chemotherapeutics and pharmacological inhibitor}

Table III.5-1 Chemotherapeutics

\begin{tabular}{lll} 
Name & Systematic name & Company \\
\hline Gemcitabine & $2^{\prime}, 2^{\prime}$-difluorodeoxycytidine $(\mathrm{dFdC})$ & $\begin{array}{l}\text { Eli Lilly, Indianapolis, IN, } \\
\text { United States }\end{array}$
\end{tabular}

Table III.5-2 Inhibitors

\begin{tabular}{llll} 
Inhibitor & Commercial name & Target & Company \\
\hline ATRi & VE-821 & ATR & Selleckchem
\end{tabular}


Chk1i

Nutlin-3

Plk1i

Roscovitine

RO-3306

Wee1i
SB-218078

Nutlin-3

GSK-461364

Roscovitine

RO-3306

MK-1775
Chk1

Mdm2

Plk1

Cdk1, 2 and 5 Cell Signaling

Cdk1

Wee1
Calbiochem, Merck

Sigma-Aldrich

Sigma-Aldrich

Selleck

\section{III.6 Enzymes and buffers}

Table III.6-1 Enzymes and buffers

\section{Reagent}

Buffer for M-MuLV RT, 10x

Buffer for Taq with $\mathrm{KCl}, 10 \mathrm{x}$

Buffer R

M-MuLV Reverse transcriptase (RT)

RNase A

Taq DNA polymerase (Taq)

\section{Company}

New England Biolabs, Ipswich, MA, United States

Fermentas, Thermo Scientific

Fermentas, Thermo Scientific

New England Biolabs

Qiagen, Venlo, Netherlands

Fermentas, Thermo Scientific

\section{III.7 Kits}

Table III.7-1 Kits

Name

Guava Check Kit

Immobilon Western HRP Substrate Peroxide Solution

PureYield Plasmid Midiprep System

SuperSignal West Femto Maximum Sensitivity Substrate

\section{Company}

Millipore, Merck

Millipore, Merck

Promega

Thermo Scientific

\section{III.8 Oligonucleotides}

Table III.8-1 Small interfering RNAs

Name (identifies target) siRNA ID

Negative Control No. 1 Undisclosed

Negative Control No. 2 Undisclosed

ATR \#1

ATR \#2

s57270

Chk1 \#1

s56824

Chk1 \#2

s504

Wee1 \#1

s503

Wee1 \#2

s21

Mus81

$\mathrm{s} 22$

Claspin \#1

s37038

Claspin \#2

s34330

s34331 
All siRNAs are Silencer Select from Ambion, Life Technologies.

Table III.8-2 Primers

\begin{tabular}{lll} 
Name & Sequence & Application \\
\hline anchored oligo-dT & $\mathrm{dT}_{23}$ VN & RT-PCR \\
random nonamer & 5'-NNNNNNNNN-3' & RT-PCR \\
36B4 forward & 5'-GATTGGCTACCCAACTGTTG-3' & \\
36B4 reverse & 5'-CAGGGGCAGCAGCCACAAA -3' & qPCR \\
ATR forward & 5'-CATGCTAACAGGTCCGAGT -3' & \\
ATR reverse & 5'-GTCCCAGTCTGACACTCCA -3' & qPCR
\end{tabular}

III.9 Antibodies

Table III.9-1 Primary antibodies

\begin{tabular}{|c|c|c|c|c|}
\hline Target & Clone & $\begin{array}{l}\text { Source } \\
\text { organism }\end{array}$ & $\begin{array}{l}\text { Dilution for } \\
\text { immunoblotting }\end{array}$ & Company \\
\hline ATR & $\mathrm{N}-19$ & goat & $1: 300$ & $\begin{array}{l}\text { Santa Cruz Biotechnology, } \\
\text { Santa Cruz, CA, United States }\end{array}$ \\
\hline ATR pT1989 & & rabbit & $1: 300$ & Kerafast \\
\hline Cdc2 & $\mathrm{POH}-1$ & mouse & $1: 2,000$ & $\begin{array}{l}\text { Cell Signaling Technology, } \\
\text { Beverly, MA, United States }\end{array}$ \\
\hline Cdc2 pY15 & & rabbit & $1: 1,000$ & Abcam \\
\hline Chk1 & 2G1D5 & mouse & $1: 1,000$ & Cell Signaling Technology \\
\hline Chk1 pS317 & & rabbit & $1: 1,000$ & Cell Signaling Technology \\
\hline Claspin & & rabbit & $1: 1,000$ & Cell Signaling Technology \\
\hline H2AX pS319 & JBW301 & mouse & $1: 4,000$ & Millipore, Merck \\
\hline H2AX pS319 & & rabbit & $1: 1,000$ & Cell Signaling Technology \\
\hline H3 pS10 & $\begin{array}{l}(\mathrm{D} 2 \mathrm{C} 8) \\
\mathrm{XP}\end{array}$ & rabbit & $1: 1,600$ & Cell Signaling Technology \\
\hline HSC70 & B-6 & mouse & $1: 15,000$ & Santa Cruz Biotechnology \\
\hline Mus81 & $\begin{array}{l}\text { MTA30 } \\
2 \mathrm{G} 10 / 3\end{array}$ & mouse & $1: 500$ & Santa Cruz Biotechnology \\
\hline Mdm2 & $\begin{array}{l}(\mathrm{Ab}-1) \\
\text { IF-2 }\end{array}$ & mouse & $1: 300$ & Calbiochem \\
\hline p21 & $\begin{array}{l}(\mathrm{Ab}-1) \\
\text { EA10 }\end{array}$ & mouse & $1: 500$ & Calbiochem \\
\hline p53 & DO-1 & mouse & $1: 1,000$ & Santa Cruz Biotechnology \\
\hline PARP & & rabbit & $1: 1,000$ & Cell Signaling Technology \\
\hline Plk1 & $35-206$ & mouse & $1: 1,000$ & Life Technologies \\
\hline Plk1 pT210 & K50-483 & mouse & $1: 1,000$ & BD Pharmigen \\
\hline
\end{tabular}




$\begin{array}{lllll}\text { Rad17 } & \text { H-3 } & \text { mouse } & 1: 1,000 & \text { Santa Cruz Biotechnology } \\ \text { Rad17 pS645 } & \text { D5H5 } & \text { rabbit } & 1: 1,000 & \text { Cell Signaling Technology } \\ \text { Wee1 } & & \text { rabbit } & 1: 1,000 & \text { Cell Signaling Technology } \\ \text { B-Actin } & \text { AC-15 } & \text { mouse } & 1: 20,000 & \text { Abcam }\end{array}$

Table III.9-2 Secondary antibodies

\begin{tabular}{|c|c|c|}
\hline Antibody & Cat. Number & Company \\
\hline Alexa-Fluor-488 goat anti rabbit & A-11034 & Invitrogen, Life Technologies \\
\hline Alexa-Fluor-546 goat anti mouse & A-11003 & Invitrogen, Life Technologies \\
\hline Alexa-Fluor-594 goat anti mouse & A-11005 & Invitrogen, Life Technologies \\
\hline $\begin{array}{l}\text { HRP-coupled AffiniPure } F\left(a b^{\prime}\right) 2 \\
\text { fragment, anti-mouse } \lg G(H+L)\end{array}$ & $711-036-152$ & $\begin{array}{l}\text { Jackson Immunoresearch, } \\
\text { Europe, Newmarket, UK }\end{array}$ \\
\hline $\begin{array}{l}\text { HRP-coupled AffiniPure } \quad F\left(a b^{\prime}\right) 2 \\
\text { fragment, anti-rabbit } \lg G(\mathrm{H}+\mathrm{L})\end{array}$ & $715-036-150$ & Jackson, Immunoresearch \\
\hline HRP-coupled AffiniPure, anti-goat & $115-035-044$ & Jackson, Immunoresearch \\
\hline
\end{tabular}

\section{III.10 Human cell culture}

Table III.10-1 Human cell lines

\begin{tabular}{ll} 
Cell line & Origin \\
\hline BxPC-3 & Pancreatic adenocarcinoma \\
HCT116 (wild-type p53/ p53-/-) & Colorectal carcinoma \\
HeLa & Cervical adenocarcinoma \\
MCF7 & Breast adenocarcinoma \\
MIA PaCa-2 & Pancreatic adenocarcinoma \\
PANC-1 & Pancreatic epithelioid carcinoma \\
U2OS & Osteosarcoma
\end{tabular}

Table III.10-2 Media and reagents for eukaryotic cell culture

\begin{tabular}{l} 
Reagent \\
\hline Ciprofloxacin \\
Dulbecco's Modified Eagle Medium (DMEM), powder \\
DMEM, High Glucose, Phenol-Red Free \\
Fetal Calf Serum (FCS) \\
L-Glutamine \\
McCoy's Medium \\
PBS (tablets) \\
Penicillin/Streptomycin \\
Tetracyclin \\
Trypsin/EDTA
\end{tabular}

Company

Bayer

Gibco, Life Technologies

Gibco, Life Technologies

Gibco, Life Technologies

Gibco, Life Technologies

Gibco, Life Technologies

Gibco, Life Technologies

Gibco, Life Technologies

Gibco, Life Technologies

Gibco, Life Technologies 


\section{DMEM}

DMEM, powder

$10.0 \mathrm{~g}$

$\mathrm{NaHCO}_{3}$

$3.7 \mathrm{~g}$

HEPES

$5.96 \mathrm{~g}$

dissolved in $\mathrm{H}_{2} \mathrm{O}$

\section{III.11 Software}

\section{Table III.11-1 Software}

\begin{tabular}{ll} 
Name & Company \\
\hline BD Pathway Software & Becton Dickinson \\
Celigo Software & Cyntellect \\
CFX Manager Software for & Bio-Rad \\
qPCR cycler & \\
Excel & Microsoft, Redmond, WA, United States \\
Guava Express Software & Millipore, Merck \\
INTAS lab ID & Intas Science Imaging Instruments \\
NanoDrop Software & Peqlab \\
Adobe Photoshop CS5 & Adobe Systems, San Jose, CA, United States
\end{tabular}




\section{Methods}

\section{IV.1 Cell Biology Techniques}

\section{IV.1.1 Culturing of Human cancer cell lines}

All the cell culture work was performed under the hood in sterile conditions. 1X PBS, $0.1 \%$ trypsin/EDTA and medium were pre-warmed prior to use.

Cell lines were adherent and cultured either in sterile tissue culture petri-dishes or flasks with suitable media. Media of the cell lines was supplemented with $10 \%$ FCS, $200 \mu \mathrm{M} \mathrm{L-}$ glutamine and antibiotics - 50U/ml Penicillin and Streptomycin, 20 $\mu \mathrm{g} / \mathrm{ml}$ Tetracycline and $10 \mu \mathrm{g} / \mathrm{ml}$ Ciprofloxacin; depending on the tolerance capacity of the cell lines (Table IV.1-1).

Table IV.1-1 Media for different cell lines

\begin{tabular}{lll} 
Cell lines & Media & Supplements \\
\hline U2OS (Osteocarcinoma) & DMEM & All \\
$\begin{array}{l}\text { Panc1 (Pancreatic tumor) } \\
\text { MiaPaCa2 DMEM }\end{array}$ & All \\
$\begin{array}{l}\text { tumor) } \\
\text { BxPC3 (Pancreatic tumor) }\end{array}$ & DMEM & All except Ciprofloxacin and Tetracycline \\
MCF7 (Breast tumor) & DMEM & All except Glutamine, Ciprofloxacin and \\
& (high & Tetracycline \\
HeLa (Cervical cancer) & glucose) & \\
HCT116 wild type p53 & DMEM & All \\
HCT116 p53-I- & Mc Coy's & All except Tetracycline \\
\hline
\end{tabular}

For the maintenance of cell lines in cell culture, they were stored at $37^{\circ} \mathrm{C}, 5 \% \mathrm{CO}_{2}$ in humidified conditions. For sub-culturing, cells were washed with $1 \mathrm{X}$ PBS to remove dead cells, trypsinized and upon detachment of the cells from the surface of the petri-dish, medium was added to stop the reaction. Cells were then re-seeded in the appropriate ratio (such as 1:2, 1:4 or so on). 


\section{IV.1.2 Freezing of cells}

Cells with low passage number were frozen for long-term storage. Freezing medium was prepared using FCS and DMSO in the ratio of 9:1. It was allowed to cool. Cells were washed and trypsinized, followed by addition of media to stop this reaction. Cells were then centrifuged at $1000 \mathrm{rpm}$ for $5 \mathrm{~min}$, media was removed, and they were re-suspended in freezing medium and immediately transferred to cryotubes (on ice). They were first stored at $-80^{\circ} \mathrm{C}$ for $24 \mathrm{~h}$ and transferred to liquid nitrogen afterwards.

From a large dish $(25 \mathrm{~cm}), 3-4$ aliquots of $1 \mathrm{ml}$ can be prepared.

\section{IV.1.3 Thawing of cells}

Frozen cells were thawed at $37^{\circ} \mathrm{C}$ for a few seconds and added into medium. They were centrifuged at $1000 \mathrm{rpm}$ for $5 \mathrm{~min}$, resuspended in fresh medium and seeded in a $10 \mathrm{~cm}$ petri-dish. Medium in the plate was changed after $24 \mathrm{~h}$.

Cells were used for transfection after passaging 3-4 times.

\section{IV.1.4 Transfection of cells with siRNA}

To knockdown specific gene of interest, reverse transfection was performed wherein cells were seeded on the same day as transfection mix was added.

Cells were washed with 1 X PBS, trypsinized and afterwards media was added to make a cell suspension. Cells were counted on a Neubauers chamber slide.

Using following formula, concentration of cells was calculated:

Concentration of cells $($ cells $/ \mathrm{ml})=$ Number of cells $X 10,000$

\section{Number of squares}


siRNA and Lipofectamine (LF2000) were diluted in the medium (without medium) as mentioned in the Table IV.1-2.

Table IV.1-2 siRNA and lipofectamine dilution according to plate format

\begin{tabular}{llllll}
$\begin{array}{l}\text { Plate } \\
\text { Format }\end{array}$ & $\begin{array}{l}\text { Cell number* } \\
(\mathbf{U} 20 \mathrm{~S})\end{array}$ & $\begin{array}{l}\text { Medium } \\
(\boldsymbol{\mu l})\end{array}$ & $\begin{array}{l}\text { siRNA } \\
\mathbf{( 5 0} \boldsymbol{\mu M})\end{array}$ & $\begin{array}{l}\text { Medium } \\
(\boldsymbol{\mu l})\end{array}$ & $\begin{array}{l}\text { LF2000 } \\
(\boldsymbol{\mu l})\end{array}$ \\
\hline $\mathbf{9 6}$ well & 8,000 & 25 & 4,5 pmol (silencer siRNA) & 14,75 & 0,25 \\
$\mathbf{9 6}$ well & 8,000 & 25 & $\begin{array}{l}1,5 \quad \text { pmol (silencer select } \\
\text { siRNA) }\end{array}$ & 14,75 & 0,25 \\
$\mathbf{1 2}$ well & $1,00,000$ & 100 & $1 \mu \mathrm{l}(50 \mathrm{pmol})$ & 100 & 2 \\
$\mathbf{6}$ well & $1,60,000-$ & 200 & $2 \mu \mathrm{l}(100 \mathrm{pmol})$ & 200 & 4
\end{tabular}

${ }^{\star}$ Cell number can vary depending on the cell line.

Diluted Lipofectamine was incubated for $5 \mathrm{~min}$ at room temperature; appropriate amount of it was then mixed with diluted siRNA and incubated for 20 min. After incubation, siRNAlipofectamine mix was pipetted into the wells, followed by addition of suitable amount of cells from cell suspension.

Media was changed after $24 \mathrm{~h}$ and depending on the experimental setup; cells were either harvested or treated with required chemicals or drugs.

\section{IV.1.5 Chemical or drug treatment}

Stock solutions of the chemicals or drugs were prepared either in water or DMSO. Required amount from stock solution was dissolved in medium to get the final concentration as outlined in the Table IV.1-3.

Table IV.1-3 Concentration of chemicals or drugs used in the treatment

\begin{tabular}{lllll} 
Inhibitor & Target & Solvent & $\begin{array}{l}\text { Stock } \\
\text { concentration }\end{array}$ & $\begin{array}{l}\text { Working } \\
\text { concentration }\end{array}$ \\
\hline SB 218078 & Chk1 & DMSO & $2.5 \mathrm{mM}$ & $2.5 \mu \mathrm{M} / 5 \mu \mathrm{M}$ \\
VE-821 & ATR & DMSO & $10 \mathrm{mM}$ & $10 \mu \mathrm{M} / 5 \mu \mathrm{M}$ \\
MK-1775 & Wee1 & DMSO & $1 \mathrm{mM}$ & $1 \mu \mathrm{M} / 0.5 \mu \mathrm{M}$ \\
Roscovitine & CDK1,-2 and -5 & DMSO & $20 \mathrm{mM}$ & $20 \mu \mathrm{M}$ \\
RO-3306 & CDK1 & DMSO & $10 \mathrm{mM}$ & $10 \mu \mathrm{M}$ \\
GSK 461364 & Plk1 & DMSO & $10 \mathrm{mM}$ & $100 \mathrm{nM}$ \\
Nutlin-3 & Mdm2 & DMSO & $20 \mathrm{mM}$ & $8 \mu \mathrm{M}$ \\
\hline
\end{tabular}




\begin{tabular}{llll}
\hline $\begin{array}{l}\text { Chemotherapeutic } \\
\text { Drug }\end{array}$ & Solvent & $\begin{array}{l}\text { Stock } \\
\text { concentration }\end{array}$ & $\begin{array}{l}\text { Working } \\
\text { concentration }\end{array}$ \\
\hline Gemcitabine & Water & $64 \mathrm{mM}$ & $300 \mathrm{nM} / 25 \mathrm{nM} /$ \\
& & $5 \mathrm{nM}$ \\
\hline
\end{tabular}

\section{IV.1.6 Cell proliferation assay}

To monitor the health and growth rate of the cells, the fundamental tool is to assess the proliferative activity of the cells grown in culture. To track the cell proliferation Celigo cell cytometer was used; it can be used to measure the confluency or perform direct cell counting of the cells in the plates (compatible with the instrument). It provides a nondestructive, label-free and automated way of measuring the cell growth upon desired treatment of the cells.

This assay was utilized to ensure the growth of the cells when treated with inhibitors of Wee1/ Chk1/ ATR with or without gemcitabine. Cells were seeded in 96- well plate, treated after 18-24 $\mathrm{h}$ and the confluency of the cells was measured (labeled as Day0). After $24 \mathrm{~h}$, media was replaced with fresh media; measurement was taken (Day1) and subsequent measurements were made after every $24 \mathrm{~h}$ and media was changed after 48 h. Once required amount of measurements were taken, confluency was plotted against time (in days) using Microsoft Excel.

\section{IV.1.7 Protein Chemistry Techniques}

\section{IV.1.8 Preparation of whole cell lysates}

Cell lysate preparation was done on ice. Cells adherent to the surface of the plate were scraped off into the medium and transferred into an Eppendorf tube. Cells were pelleted down by centrifuging at $4000 \mathrm{rpm}$ for $3 \mathrm{~min}$ at $4^{\circ} \mathrm{C}$. Media was removed and $1 \mathrm{X}$ PBS was added for washing the cells. Cells were resuspended in appropriate amount of lysis buffer; depending on the pellet size, for a 6-well plate, 100-120 $\mu$ l while for a 12-well plate, 50-60 $\mu$ of lysis buffer was used. Cells were briefly vortexed and kept on shaking at $4^{\circ} \mathrm{C}$ for $20 \mathrm{~min}$ for efficient lysis of the cells. Cell lysate was then centrifuged at 13,000 rpm for 10min to let DNA settle down.

Bicinchoninic acid assay (BCA assay) kit was used to normalize the concentration of proteins. In this assay, total concentration of protein is exhibited by a color change of sample solution from green to purple in proportion to protein concentration, which can then be measured by colorimetric techniques. According to user's manual, BCA reagents 
were mixed in the ratio $A: B=98: 2$ and $5 \mu \mathrm{l}$ of protein were added to this mixture, incubated at $37^{\circ} \mathrm{C}$ for half an hour. Using Nanodrop spectrophotometer, a standard curve was prepared with different dilutions of BSA (provided with the kit); concentration of proteins was then measured using this standard curve. To the normalized amount of protein, 6X Laemmli buffers was added to the final concentration of $1 \mathrm{X}$ and samples were boiled at $95^{\circ} \mathrm{C}$ to reduce the disulfide bonds and denature the proteins.

\section{IV.1.9 Separation of proteins by SDS-PAGE (Sodium dodecyl sulfate- Polyacrylamide Gel electrophoresis)}

The method of SDS-PAGE, a widely used method for separating proteins based on their electrophoretic mobility was refined, in the way it is used nowadays, by Ulrich K. Laemmli (Laemmli, 1970). During cell lysate preparation, samples were boiled in Laemmli buffer, consisting of SDS as one of its component. SDS is an anionic detergent which imparts an even distribution of negative charge per unit mass of most of the proteins, thereby resulting in a fractionation by approximate size during electrophoresis. Gels that were used for the separation consisted of two layers- lower percentage of acrylamide/bisacrylamide (5\%) stacking gel layer with $\mathrm{pH} 6.8$ and higher percentage of acrylamide/bisacrylamide (varies from $6 \%-12 \%$; depending on the size of the proteins to be separated) resolving gel layer with $\mathrm{pH}$ 8.8. Stacking gel formed the upper layer having well pockets for loading the sample; it served the purpose of compressing the proteins in a thin layer before they enter to the lower layer of resolving gel. In resolving gel, actual separation of proteins according to their size took place. Components of the gels are summarized in the Table IV.1-4.

Normalized protein samples were loaded onto the gel, along with a pre-stained protein marker in a separate well pocket to track the separation and determine the size of the proteins. Gels were run at a constant voltage of $80 \mathrm{~V}$ to $120 \mathrm{~V}$ until desired separation was achieved.

Table IV.1-4 Components of stacking and resolving gels

\begin{tabular}{lll} 
& Stacking gel & Resolving gel \\
\hline Acrylamide/bisacrylamide & $5 \%$ & $6-12 \%$ \\
1M Tris, pH 6.8 & $126 \mathrm{mM}$ & - \\
1.5M Tris, pH 8.8 & - & $375 \mathrm{mM}$ \\
10\% SDS & $0.1 \%$ & $0.1 \%$ \\
10\% APS & $0.1 \%$ & $0.1 \%$ \\
TEMED & $0.3 \%$ & $0.4 \%$ \\
\hline
\end{tabular}




\section{IV.1.10Western blotting}

The technique (also known as Protein Immunoblotting) allows detection of specific proteins in a cell lysate. The method was introduced by Towbin et. al. (1979) and is now a routine technique for protein analysis. The proteins separated by electrophoresis are transferred to a nitrocellulose or polyvinylidene difluoride (PVDF) membrane. Membrane is then incubated with an antibody (called as primary antibody) against the epitope of a specific protein, followed by addition of another antibody (called as secondary antibody) which can bind to the species-specific region of the primary antibody and is conjugated to an enzyme like Horseradish peroxidase. The enzyme can convert its substrate into a product that produces luminescence, the light output is directly proportional to the amount of protein and can be captured by using film, a CCD camera or a phosphorimager designed for chemiluminescent detection.

Once proteins were separated by SDS-PAGE, a sandwich of gel and membrane was prepared for electroblotting of proteins from gel to membrane. Transfer was performed at constant voltage of $100 \mathrm{~V}$ for $120 \mathrm{~min}$ (for the transfer of big proteins, PVDF membrane was used and transfer was done at constant voltage of $40 \mathrm{~V}$ for $24 \mathrm{~h}$ ). After transfer was finished, membrane was stained with Ponceau $S$ to check whether transfer was uniform and proteins were equally loaded. For blocking the unspecific sites on the membrane, where antibodies can bind, blocking buffer was added to the membrane for $45 \mathrm{~min}$. It was followed by overnight incubation with appropriate dilution of primary antibody at $4^{\circ} \mathrm{C}$ (for more details on dilution of primary antibodies, refer to Table III.9-1), washing of the primary antibody with washing buffer (PBST or TBST) and addition of secondary antibody (1:10,000 dilution; for both primary and secondary antibody blocking buffer was used for making dilutions) for $1 \mathrm{~h}$ at room temperature. Membrane was then once washed with blocking buffer, followed by washing buffer. For visualizing the amount of protein, suitable amount of substrate solution (Immobilon Western HRP Substrate Peroxide Solution) was applied and luminescence was detected using a Chemocam HR 163200 imager. For weak signals, the more sensitive substrate solution SuperSignal West Femto Maximum Sensitivity Substrate was used.

\section{IV.1.11Immunoprecipitation}

Immunoprecipitation (also referred as IP) is the technique of precipitating a protein using antibody that specifically binds to that protein. This method can be used to isolate and concentrate a particular protein from a sample having thousands of different proteins. This approach can be used for- identifying activation status of protein, determine posttranslational modifications and to study protein-protein or protein-nucleic acid interactions. It is based on the principle that an antibody forms an immune complex with its specific 
target protein in a sample (such as cell lysate), this immune complex is then captured, or precipitated, on a beaded support to which an antibody-binding protein is immobilized (such as Protein A or G), and other proteins not precipitated on the beads are washed away. Finally, the protein is eluted from the support using denaturing buffers and analyzed by western blotting.

For endogenous Immunoprecipiation, cells were seeded in atleast a $10 \mathrm{~cm}$ petri dish. Protease inhibitors (complete (mini) inhibitor mix from Roche) and phosphatase inhibitors ( $\mathrm{Na}$ fluoride, Na pyrophosphate) were added to IP-lysis buffer just before its use. IP consists of 5 defined steps: Equilibration of beads- $50 \mu$ l per sample of 50/50 Protein $G$ sepharose beads slurry was suspended in an eppendorf tube, washed 3 times with IPlysis buffer by spinning the beads at $4000 \mathrm{rpm}$ for $2 \mathrm{~min}$ at $4^{\circ} \mathrm{C}$ and finally resuspended in $50 \mu \mathrm{l}$ of IP-lysis buffer. Beads were stored at $4^{\circ} \mathrm{C}$ for later use. Sample preparation- It was performed on ice. Media was removed and cells adhered to petri dish were washed with 5 $\mathrm{ml}$ PBS, followed by addition of $1 \mathrm{ml}$ pre-chilled IP-lysis buffer (In case, treatment of cells leads to lot of cell death, media was taken in a falcon tube, centrifuged so that cells settle down, cells washed with PBS and resuspended in IP-lysis buffer). Cell lysate in IP-lysis buffer was scraped off the plate and transferred to an Eppendorf tube; it was then homogenized by pushing 5 times with a 26G insulin syringe. Sonication of lysate was then performed in Sonication device Bioruptor (Diagenode, Liège, Belgium) at medium power for $10 \mathrm{~min}$ to destroy the DNA. After it, cell lysate was centrifuged at $13000 \mathrm{rpm}$ at $4^{\circ} \mathrm{C}$ for 15 min to get rid of cell debris and supernatant was transferred to a new eppendorf tube. Preclearing- Equilibrated beads were added to the lysate and incubated for $1 \mathrm{~h}$ at $4^{\circ} \mathrm{C}$ on a rotor followed by centrifugation at $3000 \mathrm{rpm}$ at $4^{\circ} \mathrm{C}$ for $4 \mathrm{~min}$. Supernatant was transferred into a new eppendorf, $50 \mu \mathrm{l}$ of this lysate was saved as input. Antigenantibody reaction- To the rest of the lysate, $2 \mu \mathrm{g}$ of antibody was added and incubated overnight at $4^{\circ} \mathrm{C}$ on a rotor. Antibody -beads coupling- $30 \mu \mathrm{l}$ of equilibrated beads were put in the lysates and incubated $1 \mathrm{~h}$ at $4^{\circ} \mathrm{C}$ on a rotor, which were centrifuged at $3000 \mathrm{rpm}$ for $2 \mathrm{~min}$ at $4^{\circ} \mathrm{C}$. Supernatant was discarded; pellet was washed 5 times with $800 \mu \mathrm{lIP}$ lysis buffer by spinning at $3000 \mathrm{rpm}$ for 2 min with final spin at $6000 \mathrm{rpm}$ for $2 \mathrm{~min}$. Supernatant was discarded carefully, $30 \mu \mathrm{l}$ of $6 \times$ laemmli buffer was added to the pellet and boiled at $95^{\circ} \mathrm{C}$ for $5 \mathrm{~min}$. Samples were then run on SDS-PAGE and immunoblotted.

Immunoprecipitation was performed to concentrate ATR using ATR (N-19) antibody from Santa Cruz and then immunoblotted to check the levels of phospho- ATR (T1989), which determined the activation status of ATR. 


\section{IV.1.12Immunofluorescence}

Immunofluorescence is the technique that utilizes fluorescent- labeled antibodies to detect specific target antigens, and therefore allows visualization of distribution of target antigen through the sample. More than one protein can be visualized in a single experiment using fluorescent tags that emit light at different wavelengths.

For Immunofluorescence microscopy, automated Pathway 855 (Becton Dickinson, Franklin Lakes, NJ, United States) was used which can read the fluorescence intensity in 96-well plates (Becton Dickinson). While performing the assay, media was removed and cells were fixed using $3.7 \%$ paraformaldehyde for $20 \mathrm{~min}$ at room temperature. All the following steps were performed at room temperature. Cells were then washed twice with 1X PBS, followed by permeabilization of cells with $0.5 \%$ triton-X in PBS for 15 min and blocking unspecific binding sites for 15 min using blocking solution (3\% BSA in PBS). Afterwards, primary antibody diluted in blocking solution was added for $1 \mathrm{~h}$, followed by three washes in PBS and incubation with secondary antibody (with Alexa Fluor tags) and Hoechst (for staining nucleus) diluted in blocking solution for 45 min in dark. Cells were then blocked once in blocking solution for $5 \mathrm{~min}$, washed in PBS for 3 times and suspended in PBS. The plate was covered with aluminium foil to prevent photobleaching of the fluorophore. Fluorescence was visualized and imaged under microscope.

This technique was used to measure the intensity of gammaH2AX (readout of DNA damage) within the nucleus upon combination of inhibition/removal of Wee1/ Chk1/ ATR with or without gemcitabine. Appropriate excitation wavelengths were used for taking the images. Once images were captured in automated BD pathway microscope, they were analyzed using BD Pathway software, wherein the region of interest (ROI) can be defined by Hoechst stain and software counts the ROIs and the average intensity of desired fluorophore within each ROI as well as the average intensity per well. These values can be used to plot the graph of either the median value or the average value of intensity among different treatments. 


\section{IV.2 Flow cytometric techniques}

\section{IV.2.1 Cell cycle analysis using Propidium lodide}

The method of cell cycle analysis was first described by a Van Dilla MA et.al in 1969 using Fuelgen staining, while the use of propidium iodide for cell cycle analysis was presented by Krishan A. (1975). Cell cycle analysis utilizes flow cytometry to distinguish different phases of the cell cycle. In this method, cells are permeabilized and treated with a fluorescent dye that stains DNA quantitatively (widely used dye is Propidium iodide). The fluorescence intensity of the stained cells at the emission wavelength of the dye correlates with the amount of DNA in the cells. As the cells progress in the cell cycle from G0/G1 phase to S phase, they replicate their DNA, this enables to determine the relative amount of cells in G0/G1 phase, S phase and G2/M phase because the fluorescence of cells in the G2/M phase will be twice as high as that of cells in G0/G1 phase.

Cells were seeded in 6-well plate, after 18- $24 \mathrm{~h}$, they were treated with Wee1 inhibitor in the presence or absence of gemcitabine for $24 \mathrm{~h}$ and harvested afterwards. Harvesting was done by trypsinization and all the cells, trypsinized and floating, were combined. All the steps afterwards were performed in cold condition. Cells were centrifuged at $1800 \mathrm{rpm}$ for $7 \mathrm{~min}$ and supernatant was removed. The pellet was then resuspended in $500 \mu \mathrm{l}$ of $1 \mathrm{X}$ PBS++ (PBS with additional salts) by pipetting, followed by drop wise addition of $500 \mu \mathrm{l}$ of absolute ethanol while vortexing and it was repeated twice so that final volume was $2 \mathrm{ml}$. Cells were then kept on shaking for $1 \mathrm{~min}$ and stored at $-20^{\circ} \mathrm{C}$ overnight or at least for an hour to allow fixation to occur. After fixation, cells were centrifuged at $2200 \mathrm{rpm}$ for 10 min, supernatant was removed and $1 \mathrm{ml}$ of PBS++ was added for $10 \mathrm{~min}$ to allow cells to rehydrate.Cell suspension was transferred to $1.5 \mathrm{ml}$ Eppendorf tubes. PBS++ was washed away and cells were resuspended in $300 \mu \mathrm{l}$ of $0.5 \mathrm{mg} / \mathrm{ml}$ RNAse A (preinactivated for DNAses by incubating at $70^{\circ} \mathrm{C}$ for $10 \mathrm{~min}$ ), incubated at $37^{\circ} \mathrm{C}$ for $30 \mathrm{~min}$ and depending on the density of cells more PBS was added to dilute the cells. Directly before measurement, $3 \mu \mathrm{l}$ of propidium iodide (also known as $\mathrm{PI}$, final concentration: 30 $\mu \mathrm{g} / \mathrm{ml}$ ) was added to each $100 \mu \mathrm{l}$ of cell suspension. Measurement was done in FACS machine Guava PCA-96 Base System (Millipore, Merck, Darmstadt, Germany) which detects the fluorescence intensity (corresponding to $\mathrm{Pl}$ ) from each cell and the guava software allows it to be plotted in graphical format. Percentage of cells in each phase of cell cycle was determined using the software ModFit (Verity Software House, Topsham, ME, United States). 


\section{IV.2.2 Double thymidine block for cell synchronization}

Cell synchronization improves conditions by which an actual process under scrutiny can be studied and helps clarify the linkage of the process to a particular cell cycle phase transition. Treatment with excess thymidine causes the arrest of the cells at G1/S border owing to the inhibition of DNA synthesis due to feedback inhibition of nucleotide synthesis caused by an imbalance of the nucleotide pool. Second treatment with thymidine allows the cells arrested at the late $S$ phase due to first treatment to be recovered and proceed to $\mathrm{G} 2 / \mathrm{M}$ phase pertaining them to arrest at G1/S phase of the next cycle. Therefore, most of the cells are synchronized at G1/S border using double thymidine block.

This method was used to first synchronize the cells at G1/S border and follow the effect of Wee1 inhibition with or without gemcitabine on cell cycle. Cells were seeded in 6- well plate, after $24 \mathrm{~h}$, they were treated with $2 \mathrm{mM}$ thymidine for $16 \mathrm{~h}$, followed by 4 times wash off of thymidine using fresh media. Cells were allowed to recover from arrest and proceed in the cell cycle by incubating in fresh media for $8 \mathrm{~h}$ and then treating them again with $2 \mathrm{mM}$ thymidine for another $16 \mathrm{~h}$. A well was harvested as time $0 \mathrm{~h}$ sample $(\mathrm{t}=0 \mathrm{~h})$ while others were treated with either DMSO or Wee1 inhibitor in the presence or absence of gemcitabine and harvested at different time- points. After harvesting, cells were permeabilized as mentioned in the section I.3.1 and stained for mitosis marker, MPM-2/ phospho H3. The staining is discussed in the next section I.3.3.

\section{IV.2.3 Analysis of cells in mitosis or premature mitosis}

Percentage of cells in mitosis can be determined by staining for proteins which specifically show up or are modified during mitosis. Phosphorylated Histone 3 at Ser10 (referred as, phospho $\mathrm{H} 3$ or $\mathrm{PHH} 3$ ) is a recently described immunomarker specific for cells undergoing mitosis. Mitotic cells can also be stained using MPM-2 (Mitotic Protein Monoclonal \#2) antibody, this antibody recognizes a phosphorylated epitope (phospho-[Ser/Thr]Pro) found in phospho-proteins such as MAP2, HSP70, cdc25, and DNA topoisomerase Ila, most of which are phosphorylated at the onset of mitosis. The number of phosphoproteins recognized by MPM-2 varies from species to species and with the cell type.

Premature mitosis is the phenomena where cells having incompletely duplicated DNA enter into mitosis; it can subsequently lead to mitotic catastrophe or cell death. Cells which enter into premature mitosis can be identified by staining for mitosis marker using flow cytometry. Cell population that stains positive for mitotic marker and have 2N DNA content is recognized as premature mitotic cell population.

The staining for mitosis was used to determine the percentage of cells undergoing premature mitosis after treatment with Wee1 inhibitor in combination with gemcitabine. 
Cells were seeded in 6- well plate and were either first synchronized with doublethymidine block or directly treated with Wee1 inhibitor in the presence or absence of gemcitabine. Cells were harvested and fixed as mentioned in section I.3.1. After fixation, cells were centrifuged at $2400 \mathrm{rpm}$ for $5 \mathrm{~min}$ and supernatant was removed. Cells were resuspended in $1 \mathrm{ml}$ of wash solution (0.05\% Triton-X in PBS) and cell suspension was transferred to $1.5 \mathrm{ml}$ Eppendorf tube. Cells were pelleted down by centrifuging at 2500 rpm for $5 \mathrm{~min}$ and resuspended in $70 \mu \mathrm{l}$ staining solution (2\% FCS, $0.2 \%$ Triton-X in PBS) along with appropriate dilution of either MPM-2 or phospho-H3 antibody. Cells were incubated on ice for $2 \mathrm{~h}$, followed by 2 washes with washing solution and then resuspended in $70 \mu \mathrm{l}$ of staining solution with Alexa Fluor-488 tagged secondary antibody (at 1:2000 dilution). Cells were incubated on ice in dark for $1 \mathrm{~h}$, washed once with washing solution and PBS subsequently and resuspended in $300 \mu \mathrm{l}$ of $0.5 \mathrm{mg} / \mathrm{ml}$ RNAse A solution, incubated for $30 \mathrm{~min}$ at $37^{\circ} \mathrm{C}$ and proceeded as described in section I.3.1. Samples were measured either in Guava machine (mentioned above) or FACScanto II (Becton Dickinson, Franklin Lakes, NJ, United States). Data from BD machine analyzed using the software FACSDiva (from BD) while that from Guava machine was analyzed using Guava software and percentage of cells stained positive for mitosis having 2N DNA content was determined. Figure IV-I gives an example, where cells enter into premature mitosis.

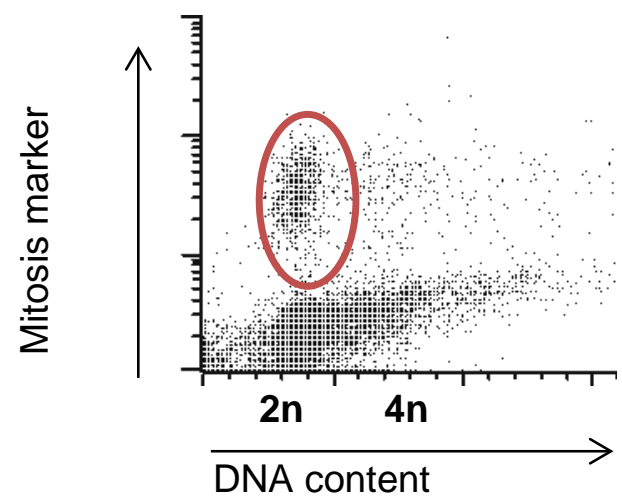

Figure IV-I Flow cytometric analysis of cells in premature mitosis.

2D graph shows the distribution of cells according to the DNA content (X-axis) and the staining for mitosis marker ( $\mathrm{Y}$ - axis). Cells outlined by red oval are premature mitotic cells. 


\section{IV.3 Real Time Quantitative Polymerase Chain Reaction (or qPCR)}

qPCR is a molecular biology technique based on Polymerase Chain Reaction (PCR) which is used to amplify as well as quantify the target DNA molecule. This technique utilizes fluorescent dye or fluorescently- tagged oligonucleotide probe for detection of the amount of DNA. It is successfully been used for quantifying the gene expression or mRNA levels.

Analysis of mRNA levels using qPCR requires following steps:

- Isolation of total RNA

- Conversion of mRNA to cDNA with Reverse transcriptase

- Quantitation of cDNA using PCR

\section{IV.3.1 Isolation of total RNA}

Total RNA from human cells was isolated using guanidinium thiocyanate-phenolchloroform extraction method. In a 6-well plate, cells were washed with $1 \mathrm{ml}$ of PBS, trypsinized and $500 \mu \mathrm{l}$ of DMEM was added. Cells were resuspended, transferred to an Eppendorf tube and kept on ice, followed by centrifugation at $2000 \mathrm{rpm}$ for $10 \mathrm{~min}$ a $4^{\circ} \mathrm{C}$. Media was removed, cells were resuspended in $1 \mathrm{ml}$ of Trizol reagent (monophasic solution of phenol and guanidinium thiocyanate) and incubated for $5 \mathrm{~min}$ to lyse cells, dissolve nucleoprotein complexes and dentaure protein. For the separation of RNA, 200 $\mu \mathrm{L}$ of chloroform was added and the samples were shaken vigorously for few seconds. After 3 min incubation at RT, phases were separated by centrifuging at $12,000 \mathrm{~g}$ for 15 min at $4^{\circ} \mathrm{C}$. RNA from the upper aqueous phase was then purified by precipitation with $500 \mu \mathrm{L}$ of isopropanol. Samples were shaken, incubated overnight at $-20^{\circ} \mathrm{C}$ and centrifuged at $12,000 \mathrm{~g}$ for $10 \mathrm{~min}$ at $4^{\circ} \mathrm{C}$. The pellet was washed with $75 \%$ ethanol. To remove any residual protein contamination, the RNA was resuspended in 50 $\mu$ l water and once more precipitated in the presence of $300 \mathrm{mM}$ sodium acetate, 1.25 times ethanol and $1 \mu \mathrm{l}$ of glycogen blue at $-80^{\circ} \mathrm{C}$ for $1 \mathrm{~h}$. The pelleted RNA was washed with $70 \%$ ethanol, air-dried, resuspended in $22 \mu \mathrm{l} \mathrm{H} 2 \mathrm{O}$ and stored at $-80^{\circ} \mathrm{C}$.

\section{IV.3.2 Conversion of mRNA to cDNA with Reverse transcriptase}

Isolated RNA was reverse transcribed with the viral M-MuLV reverse transcriptase and the use of a mixture of anchored oligo-dT primers and random nonamers. The oligo-dT primers hybridize to the poly $(A)$ tail of mRNAs while random nonamers ensure reverse transcription of RNAs without tail. 
For each reverse transcriptase (RT) reaction, $1 \mu \mathrm{g}$ of RNA was used and incubated with 2 $\mu \mathrm{L}$ of $100 \mu \mathrm{M}$ combined primers and $4 \mu \mathrm{L}$ of dNTPs (2.5 mM each) in a total volume of 16 $\mu \mathrm{L}$ for $5 \mathrm{~min}$ at $70^{\circ} \mathrm{C}$ to resolve secondary RNA structures. Then, the RT reaction mix was prepared as detailed in

Table IV.3-1 Reagents required for Reverse Transcription reactionand added to the sample. For each sample, a second RT reaction mix was prepared without reverse transcriptase to control for DNA contamination. For reverse transcription, the samples were incubated at $42^{\circ} \mathrm{C}$ for $1 \mathrm{~h}$, then heated to $95^{\circ} \mathrm{C}$ for $5 \mathrm{~min}$ to inactivate the enzyme and 20-30 $\mu \mathrm{L}$ water was added.

Table IV.3-1 Reagents required for Reverse Transcription reaction

\begin{tabular}{ll} 
Reagent & Volume $(\mu \mathrm{l})$ \\
\hline 10X RT Buffer & 2 \\
RNase Inhibitor & 0.25 \\
Reverse transcriptase & 0.125 \\
Water & 1.625 \\
\hline
\end{tabular}

\section{IV.3.3 Quantitation of CDNA using PCR}

With qPCR, the amplification of a specific DNA sequence can be monitored in real time. A fluorescent dye, such as SyBr Green, that intercalates into double-stranded DNA is used to measure the product quantity after every replication cycle. The product of gene of interest in a sample is normalized to that of a reference gene (usually any gene whose expression level is considered to be stable under the treatment conditions), and then the relative abundance of the product of gene of interest in treated sample as compared to untreated sample is calculated. To specifically amplify the cDNA of an mRNA of interest, sequence-specific primers are designed in a way that a short fragment (usually 50 to 300 bp) of the cDNA template is amplified and that they either span exon-junctions or are located in different exons. Thus, amplification of intron-containing genomic DNA can be excluded.

For the quantification of Wee1 and ATR mRNA, cDNA template levels were normalized to either GAPDH/36B4 mRNA. cDNA resulting from RT reactions without reverse transcriptase and qPCR samples without cDNA template served as controls. All samples were analyzed in triplicates. The $\mathrm{GPCR}$ reaction mix and the qPCR cycler program are detailed in Table IV.3-2 and Table IV.3-3. 
Table IV.3-2 Reaction mix for qPCR

\begin{tabular}{ll} 
Reagent & Volume $(\mu \mathrm{l})$ \\
\hline 25X qPCR reaction mix & 14 \\
Forward primer (10 pmol/ $\mu \mathrm{l})$ & 0.75 \\
Reverse primer $(10 \mathrm{pmol} / \mu \mathrm{l})$ & 0.75 \\
cDNA & 1 \\
Water & 8.5
\end{tabular}

Table IV.3-3 Cycler program for qPCR

\begin{tabular}{|c|c|c|}
\hline Temperature & Time & \\
\hline $95^{\circ} \mathrm{C}$ & $2 \min$ & \\
\hline $95^{\circ} \mathrm{C}$ & $15 \mathrm{sec}$ & \\
\hline $60^{\circ} \mathrm{C}$ & $1 \mathrm{~min}-\mathrm{read}$ & $40 x$ \\
\hline Melting curve & & \\
\hline
\end{tabular}

The fluorescence of each sample was measured once per cycle at the end of elongation ("read"). Purity of the qPCR product was controlled with a melting curve that should yield a single melting point for a specific product.

The resulting $\mathrm{Ct}$ values (amplification cycle at which the fluorescence reaches the determined threshold) were used for the calculation of the relative amount of template using the $\Delta \Delta$ Ct method, assuming $100 \%$ amplification efficiency (i.e. a product doubling with each cycle):

Relative mRNA expression $=\llbracket 2 \rrbracket^{\wedge}((\Delta \mathrm{Ct}$ ref. gene treated/target gene treated $)-(\Delta \mathrm{Ct}$ ref. gene untreated/target gene untreated))

\section{IV.4 Statistical analysis}

Statistical calculations were performed with Microsoft Excel. Statistical significance was determined using the unpaired, two-tailed student's t-test. Significance was assumed for $p$-values below 0.05. Asterisks in figures indicate resulting $p$-values as follows: ${ }^{*} p<0.05$, ${ }^{\star *} p<0.01,{ }^{* \star *} p<0.001$. n.s. $=$ not significant. $n$ in figure legends indicates the number of independent experiments. 


\section{Results}

\section{V.1 Chk1, Wee1 and ATR inhibition cooperate with gemcitabine}

\section{V.1.1 Chk1, Wee1 or ATR inhibition in combination with gemcitabine intensifies DNA damage response}

We quantified the phosphorylation of $\mathrm{H} 2 \mathrm{AX}$, referred to as $\mathrm{yH} 2 \mathrm{AX}$, upon combining the inhibition of checkpoint kinases, namely Chk1, Wee1 and ATR, with gemcitabine in different human pancreatic tumor cell lines. Cell lines used were- Panc1, MiaPaCa2, BxPC3, these cell lines have a mutated form of p53 (Deer et al. 2010, Schumacher et al. 1999). Along with these cell lines, we used U2OS, a human osteosarcoma cell line having wild-type p53.

We used pharmacological inhibitors against Chk1, Wee1 and ATR (SB218078, MK-1775, and $V E-821$ respectively) to block their activity. VE-821 is the selective and potent inhibitor of ATR (Reaper et al. 2011). SB218078 is a cell permeable, ATP-competitive, potent and selective inhibitor of checkpoint kinase (Chk1) in vitro (Jackson et al. 2000). MK-1775 selectively and potently inhibits Wee1 both in vitro and in vivo (Hirai et al. 2009). The efficiency of these inhibitors was confirmed through immunoblot staining of their respective substrates (Refer to Figure VIII-I in appendix).

Cells were treated with the inhibitors and gemcitabine for $24 \mathrm{~h}$ and afterwards analyzed for $\mathrm{y} 2 \mathrm{AX}$ intensity by quantitative immunofluorescence. We found that the inhibition of each of the three kinases cooperated with gemcitabine in potentiating the DNA damage response as evidenced by increased average $\mathrm{YH} 2 \mathrm{AX}$ intensity (Figure V-I). Inhibition of Wee1 alone also induces DNA damage response. This observation was made in all of the above-mentioned pancreatic tumor cell lines as well as osteosarcoma cell line. However, in MiaPaCa2 cells, ATR and Wee1 inhibition led to a lot of cell death resulting in loss of cells. This could be a reason for a minor increase in $\mathrm{yH} 2 \mathrm{AX}$ intensity with these two inhibitors when combined with gemcitabine. Earlier studies performed using these inhibitors have shown sensitization of tumor cells (Prevo et al. 2012), Rajeshkumar et al. 2011, Azorsa et al. 2009); however, our study focused on comparing the extent of DNA damage response upon combination of inhibition of these kinases with gemcitabine.

We also investigated the DNA damage response after transiently removing the checkpoint kinases with small interfering RNAs (siRNAs). Knockdown efficiency of siRNAs was checked through immunoblot staining of their target proteins (Refer to Figure VIII-II, 
Figure VIII-III in appendix). Cells were treated with the respective siRNAs for $48 \mathrm{~h}$, followed by $24 \mathrm{~h}$ of gemcitabine. They were fixed and analyzed for $\mathrm{\gamma H} 2 \mathrm{AX}$ intensity by immunofluorescence. We found increased gemcitabine-triggered $\mathrm{yH} 2 \mathrm{AX}$ accumulation upon Wee1 or ATR knockdown in U2OS cells and BXPC3 cells, but not with Chk1 knockdown. In Panc1 cells, a similar cooperation with Chk1 and Wee1 knockdown but not ATR knockdown was observed, while MiaPaCa2 cells were sensitized by all three knockdowns (Figure V-II).
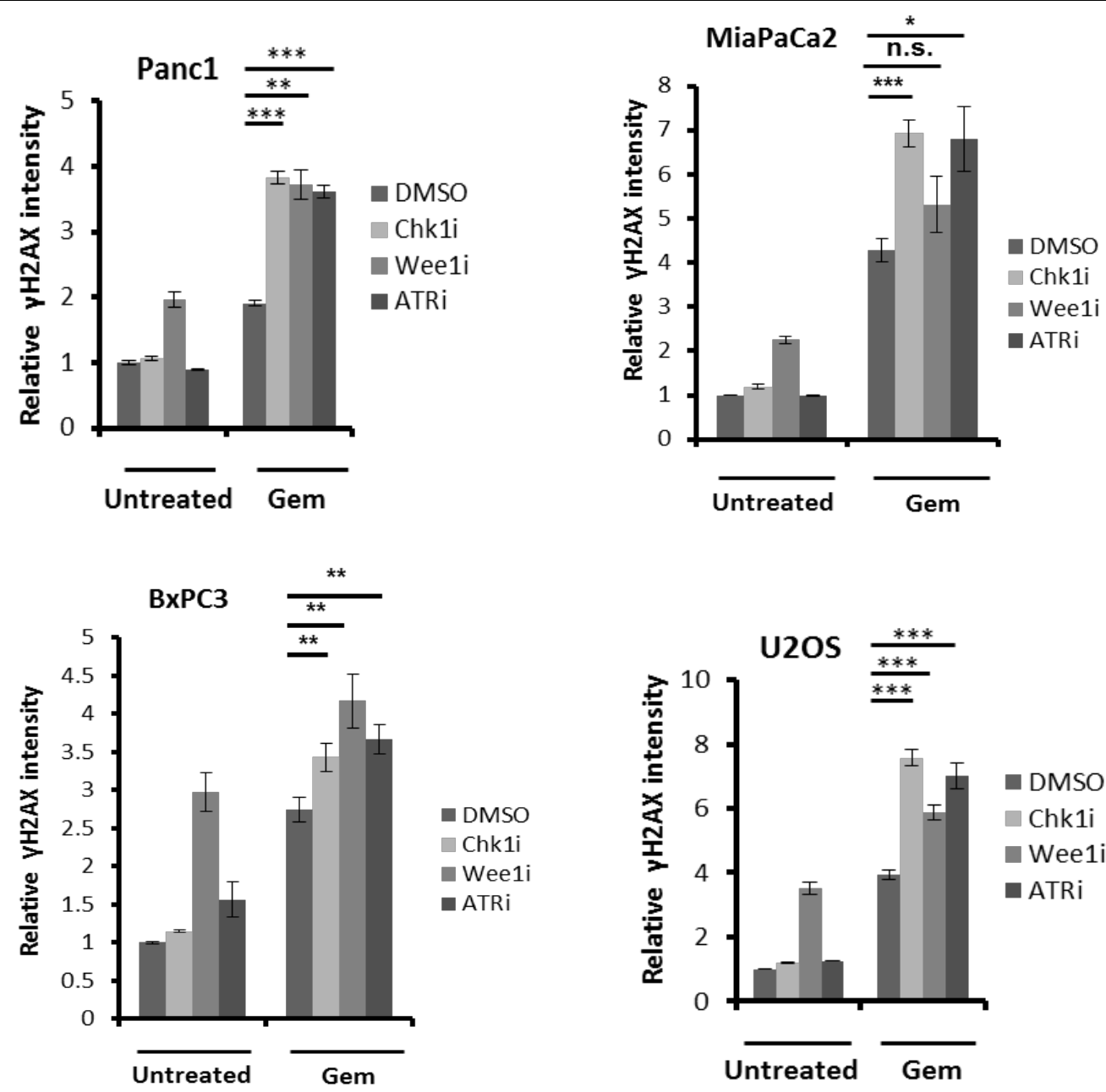

Figure V-I Three checkpoint inhibitors cooperate with gemcitabine.

Cells were treated for $24 \mathrm{~h}$ with $300 \mathrm{nM}$ gemcitabine, followed by addition of $5 \mu \mathrm{M}$ SB $218078 ; 1 \mu \mathrm{M}$

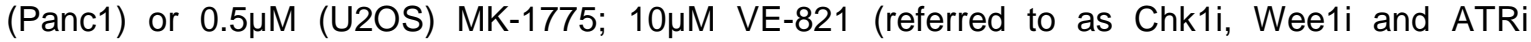
respectively, for their target kinases) in the presence of 300nM gemcitabine (Gem) for $20 \mathrm{~h}$. Cells were then fixed and stained for $\mathrm{yH} 2 \mathrm{AX}$. Measurement and analysis was done using automated immunofluorescence microscopy (BD Pathway). Error bars represent the SD, $n=3$.

These results show that the sensitization of cells by knockdown of the checkpoint kinases, in combination with gemcitabine, is dependent on cell type. This might be due to presence of different isoforms of the protein in different cell line or the knockdown induces cell death to an extent that overshadows the response of cells towards gemcitabine (as is the 
case with Chk1 knockdown in U2OS and BxPC3 cells). Wee1 knockdown with one of the siRNAs shows more $\mathrm{YH} 2 \mathrm{AX}$ intensity since this siRNA was more efficient in removing the protein (Figure V-II, Figure VIII-II).
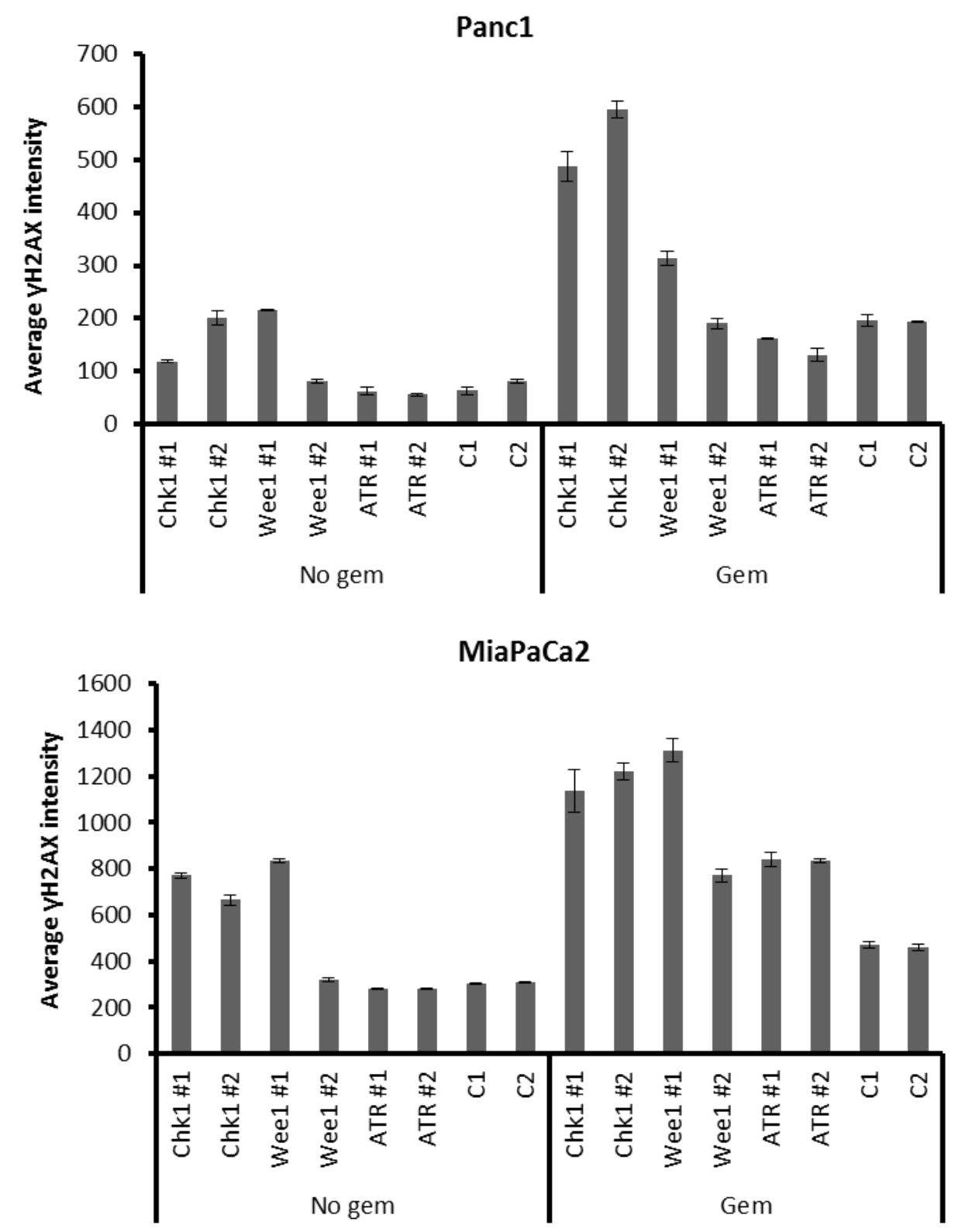


\section{BxPC3}
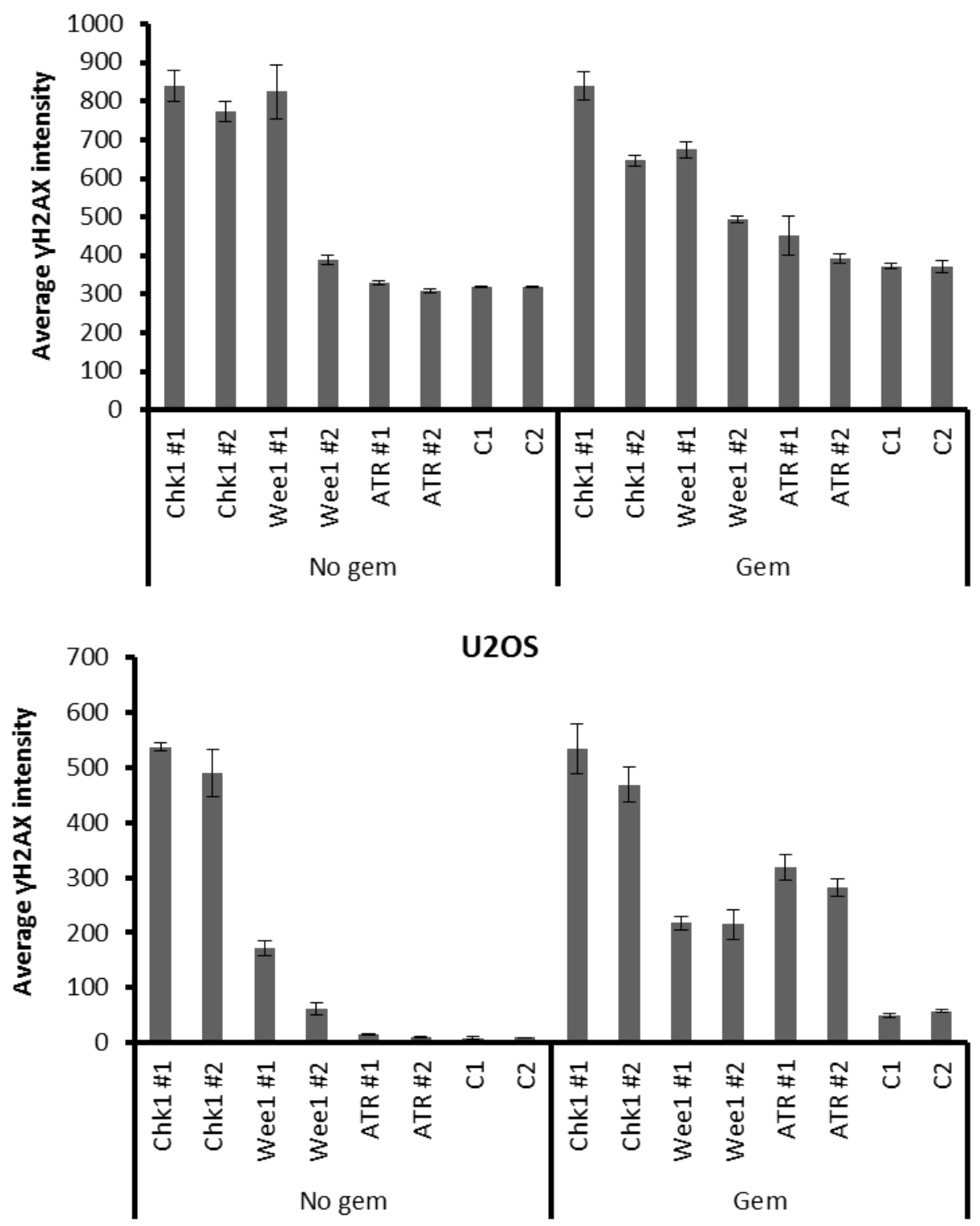

Figure V-II Depletion of the checkpoint kinases sensitize cells towards gemcitabine.

Cells were transfected with 10nM siRNAs for $48 \mathrm{~h}$, followed by $24 \mathrm{~h}$ of $300 \mathrm{nM}$ gemcitabine; they were fixed and analyzed for $\mathrm{YH} 2 \mathrm{AX}$ intensity by immunofluorescence. Error bars represent the SD, $\mathrm{n}=3$. $\mathrm{C} 1$ and $\mathrm{C} 2$ are negative control \#1 and \#2 siRNAs respectively. Left panel labeled as 'No gem' represent cells not treated with gemcitabine.

\section{V.1.2 Cell growth retards upon combination of Chk1, Wee1 or ATR inhibition with gemcitabine}

The combination of the above-mentioned inhibitors with gemcitabine increased the DNA damage response after $24 \mathrm{~h}$; however, we were interested to investigate the long-term effect of the combination treatment by following the growth of the cells over a period of time. Cells were treated with the drugs in the presence or absence of gemcitabine for $24 \mathrm{~h}$ and the growth of the cells was followed using a Celigo cytometer for 10-13 days 
(depending on the survival of the cells). The results imply that combining Wee1 or ATR inhibitor with gemcitabine retards the growth of the cells (irrespective of the cell line) to a much higher extent than Chk1 inhibitor in Panc1 and MiaPaCa2 cells. In Panc1 cells, Chk1 inhibition even promotes the cell growth to some extent. In U2OS cells, all the inhibitors in combination with gemcitabine retard the proliferation (Figure V-III).

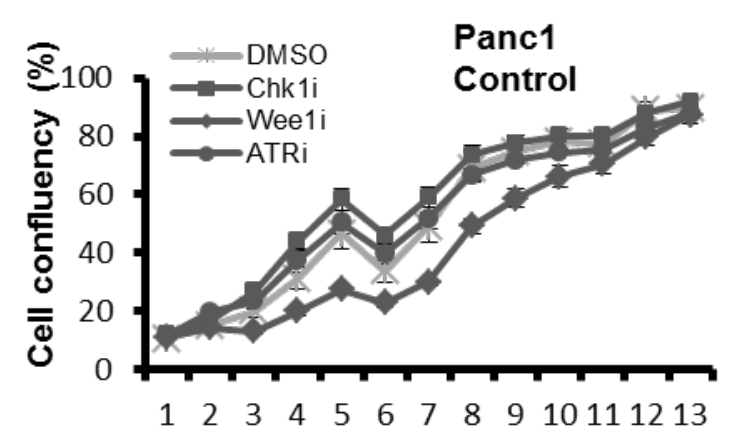

Time (days)
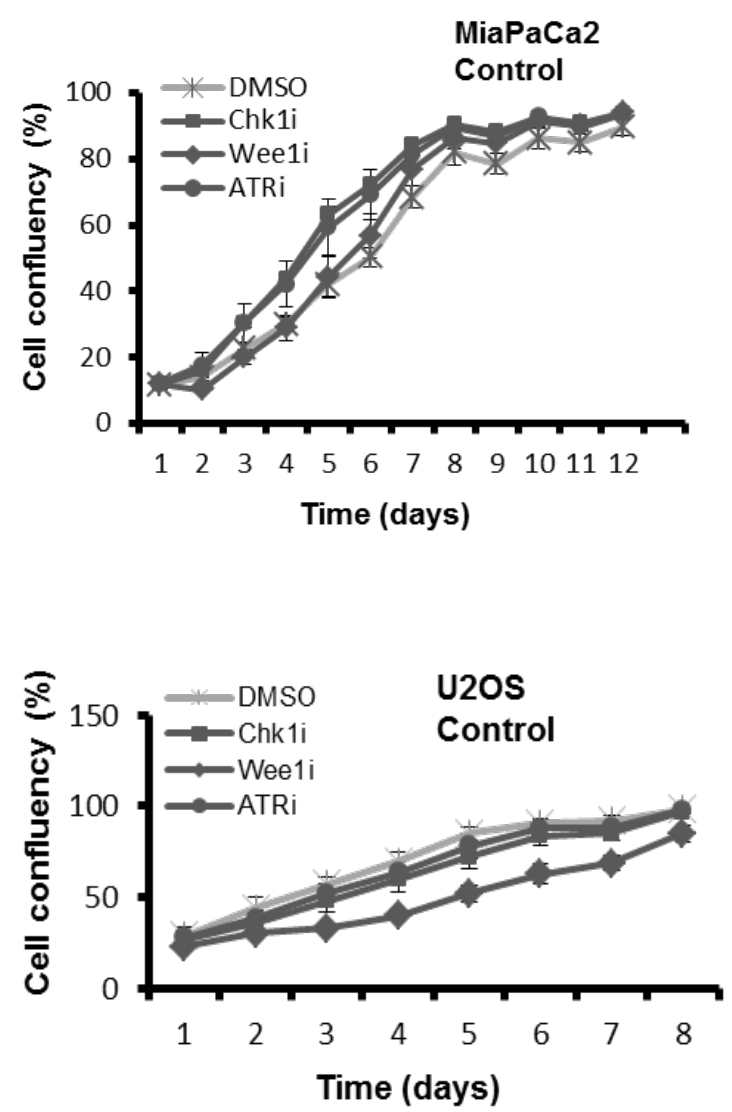
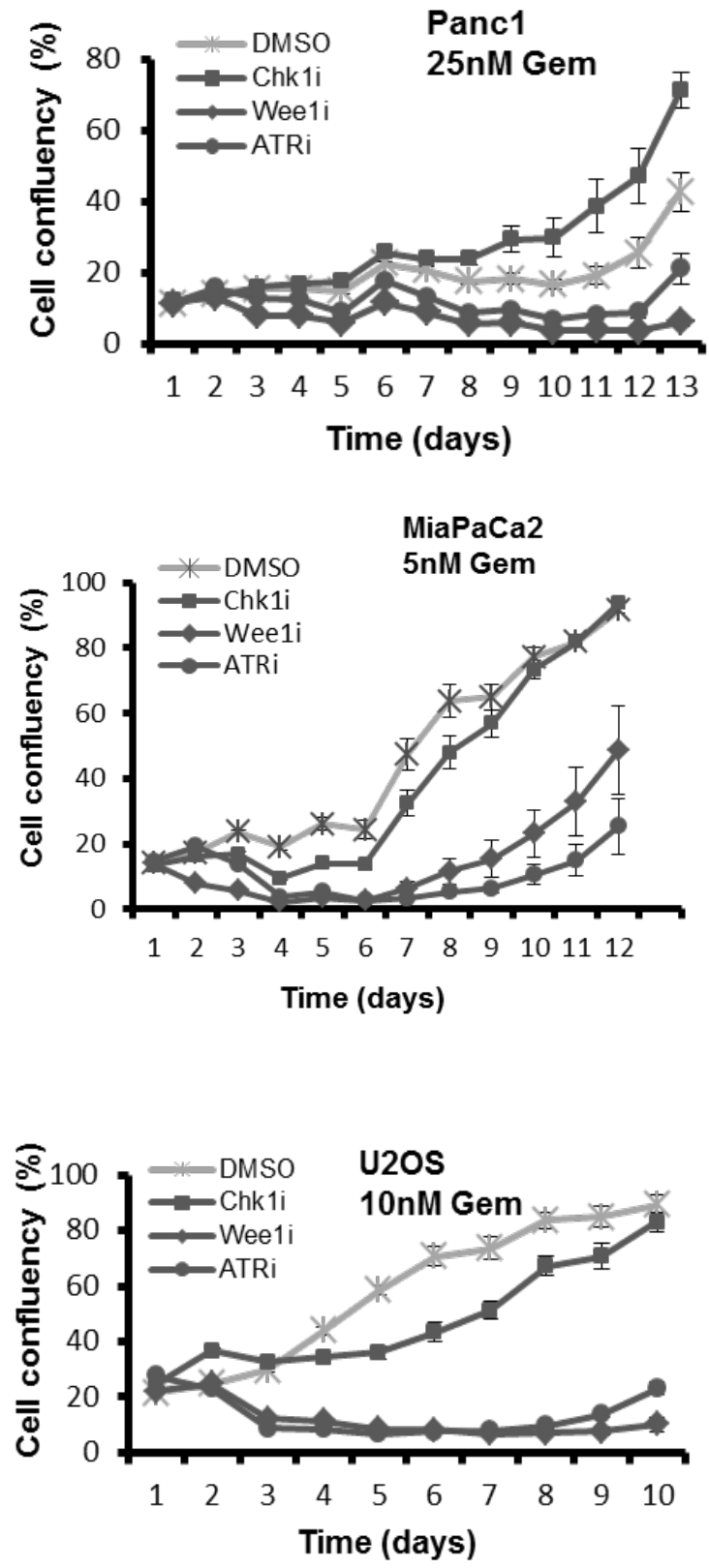

Figure V-III Long-term survivability of cells decreases upon combination of Wee1 or ATR inhibitor with gemcitabine.

Cells were treated with $2.5 \mu \mathrm{M}$ Chki, $0.5 \mu \mathrm{M}$ Wee1i and $5 \mu \mathrm{M}$ ATRi in the absence (Control) or presence of gemcitabine (Gem) at the concentrations indicated in the figure. After $24 \mathrm{~h}$, all drugs were removed and fresh medium was added. Cells were incubated for 8-13 days and confluency was measured each day using brightfield microscopy (Celigo cell cytometer). Error bars represent the SD, $\mathrm{n}=3$. 
From the above experiments, we deduce that the Wee1 inhibitor has a high potency to sensitize pancreatic tumor cells.

\section{V.2 Wee1 inhibition inactivates the ATR-Chk1 pathway}

\section{V.2.1 Inhibition or removal of Wee1 in the context of gemcitabine treatment leads to a decrease in Chk1 activation}

To analyze the signaling pathways involved in the DNA damage response upon Wee1 inhibition, we detected DNA damage signaling intermediates, apart from $\mathrm{yH} 2 \mathrm{AX}$, through immunoblotting. Cells were treated with inhibitor in the presence or absence of gemcitabine for $24 \mathrm{~h}$ and cell lysates were analyzed for the activation of DNA damage response proteins. The activity of the inhibitor was verified by staining for phosphorylation of Cdk1 at Tyr15 (Parker and Piwnica-Worms 1992); as expected this phosphorylation was decreased upon treatment with Wee1 inhibitor (Figure V-IV(a)). Phosphorylation of Chk1 at Ser317 is mediated by ATR which activates Chk1 (Hui Zhao and Piwnica-Worms 2001). It was observed that Chk1 phosphorylation (Ser317) decreased upon combination of Wee1 inhibitor with gemcitabine. Total levels of Chk1 in U2OS cells were slightly decreased which might be due to p53 activation in response to DNA damage that downregulates Chk1 expression (Gottifredi et al. 2001). Regulation of Wee1 by Chk1 has been studied, and Chk1 phosphorylates Wee1 to inhibit Cdc2 phosphorylation at Tyr15 (O'Connell et al. 1997). However, there is no previous report showing that Wee1 controls Chk1 phosphorylation or its activation. Gemcitabine is a nucleoside analog that causes replicative stress leading to activation of ATR. Chk1 is phosphorylated and activated by ATR in response to DNA damage; we speculated that the ATR-Chk1 pathway might be compromised upon combination of Wee1 inhibition with gemcitabine. In contrast, the YH2AX intensity did not decrease. We speculated that ATM or DNA-PK might be responsible for maintaining the levels of $\mathrm{yH} 2 \mathrm{AX}$.

Besides Wee1 inhibition, we also performed transient knockdown of Wee1 and observed a reduction in phosphorylation of Chk1 when combined with gemcitabine in both U2OS and Panc1 cells. The relative decrease in phospho-Chk1 as compared to total Chk1 protein was more upon Wee1 knockdown than control (Figure V-IV(b)).

We performed quantitative immunofluorescence analysis to check the phosphorylation of Rad17 at Ser645, another ATR substrate, upon combining the inhibition of checkpoint kinases with gemcitabine. Cells were treated with the $1 \mu \mathrm{M}$ Wee1 inhibitor and gemcitabine for $24 \mathrm{~h}$ and afterwards analyzed for phospho-Rad17 intensity. We found that the inhibition of Wee1 with gemcitabine decreases phospho-Rad17 intensity (Figure V-IV(c)). 
(a)

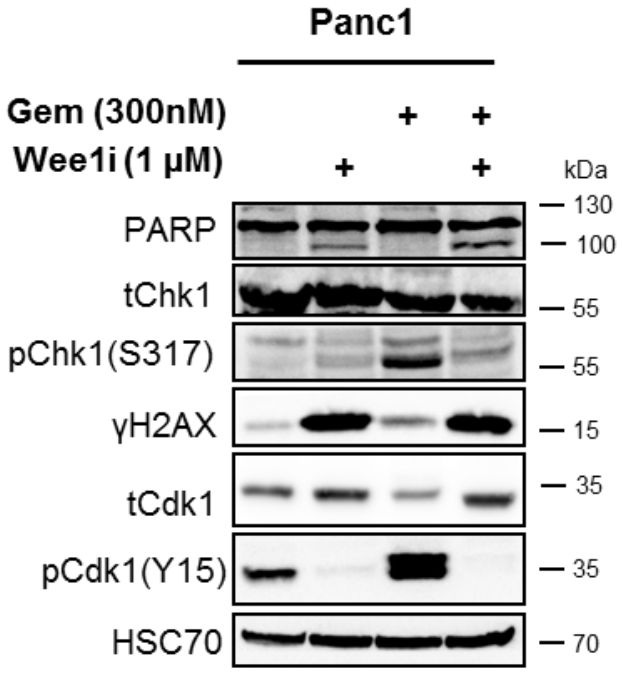

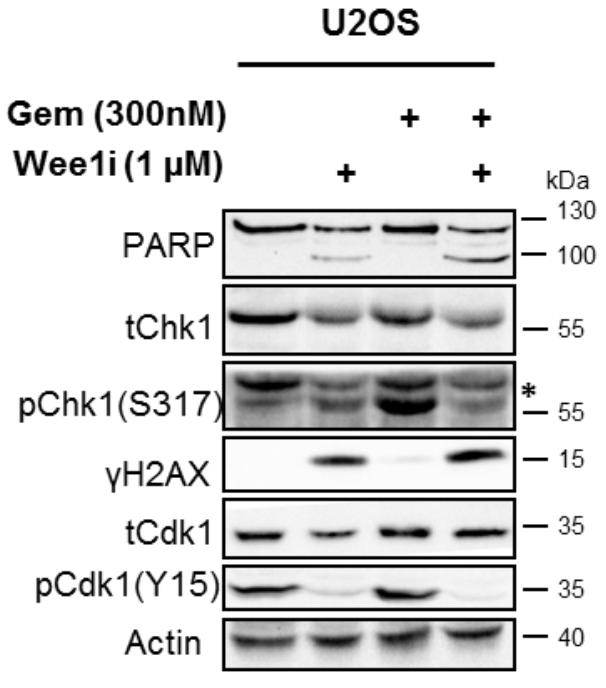

(c)

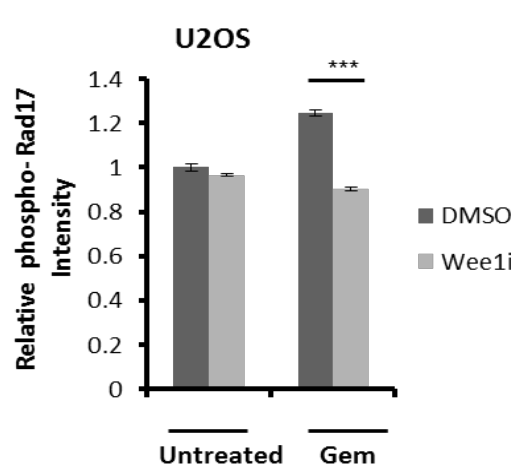

0.18

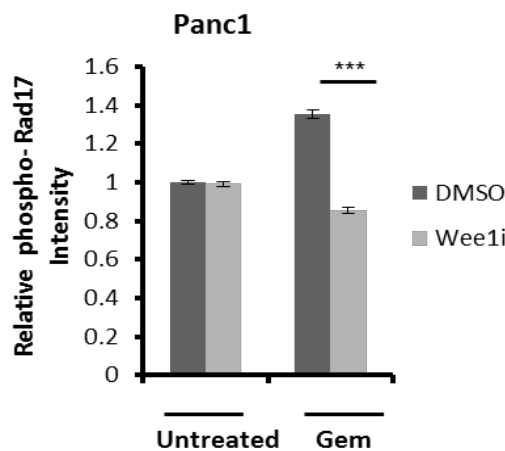

Figure V-IV Wee1 inhibition or knockdown in combination with gemcitabine, reduces Chk1 activation.

U2OS and Panc1 cells were treated with $1 \mu \mathrm{M}$ Wee1 inhibitor -MK1775 (referred to as Wee1i) and $300 \mathrm{nM}$ gemcitabine (Gem), after $24 \mathrm{~h}$ cells were harvested and cell lysate was immunoblotted. The substrate of ATR, Chk1 was analyzed for its phosphorylation. tChk1, tCdk1 stands for the total proteins while pChk1, pCdk1 for phosphorylated forms. (b) Cells were made deficient of Wee1 by transfecting with siRNA (Wee1 \#1) for $48 \mathrm{~h}$, followed by gemcitabine treatment for $24 \mathrm{~h}$ and proceeded as in (a). Cells transfected with siRNA negative control \#1 were used as control (Cntrl). Relative density represents the ratio of protein intensities in Wee1 knockdown to control. (c) Cells were treated as mentioned in (a), after $24 \mathrm{~h}$ cells were fixed and analyzed by automated immunofluorescence microscopy. Error bars represent SD, $n=3$. 
We conclude that inhibition of Wee1 in the presence of gemcitabine hampers the ATRChk1 signaling pathway and leads to inactivation of Chk1 and Rad17.

\section{V.2.2 ATR activation is hampered when Wee1 inhibition is combined with gemcitabine}

To address whether Wee1 inhibition, when combined with gemcitabine, leads to the inactivation of ATR, we detected ATR phosphorylation at Thr1989; phosphorylation of this site has earlier been described as a marker of ATR activity (Nam et al. 2011). ATR was immunoprecipitated to concentrate the protein and then immunoblotted to detect phospho-ATR (Thr1989). Phospho-ATR levels, as expected, were increased upon gemcitabine treatment, but when gemcitabine was combined with Wee1 inhibitor, the levels of this protein were decreased (Figure V-V), suggesting impaired activity of ATR. This decreased activation of ATR was independent of the p53 status of the cells, as both U2OS and Panc1 cells showed reduction of phospho-ATR upon Wee1 inhibition.

These results suggest that Wee1 governs the activation of the ATR-Chk1 pathway upon induction of DNA damage by gemcitabine.
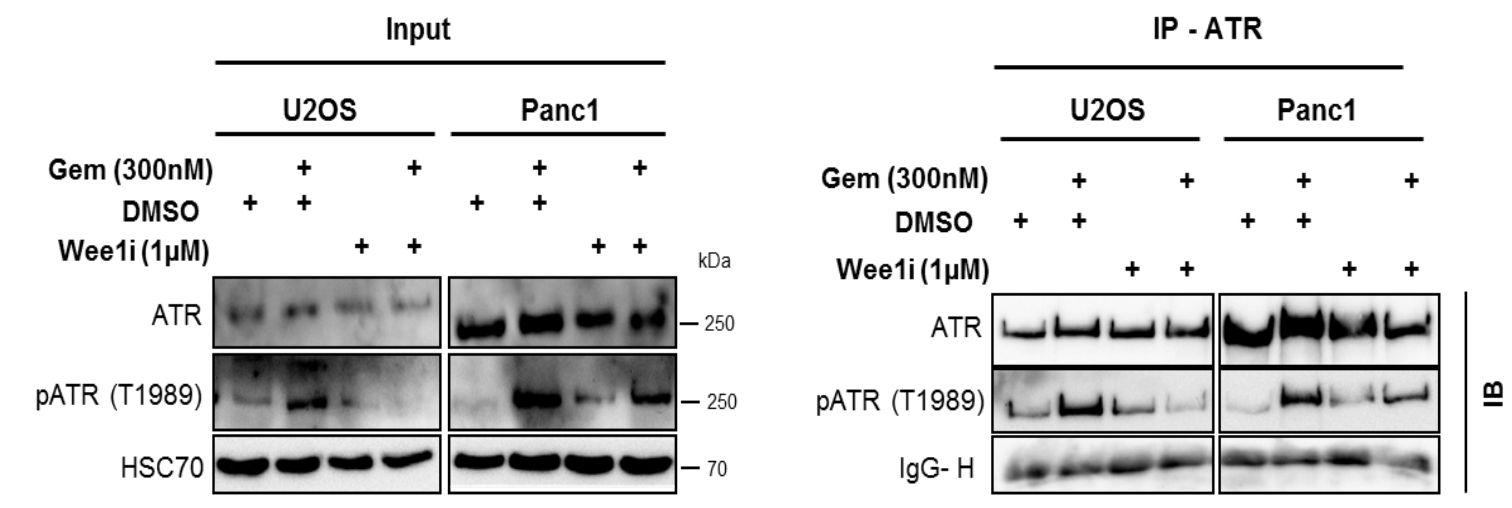

Figure V-V ATR activity decreases upon combination of Wee1 inhibitor and gemcitabine. Cells were treated with $1 \mu \mathrm{M}$ Wee1 inhibitor (MK1775) and 300nM gemcitabine for 24h. Cells were harvested and cell lysate was prepared, ATR was immunoprecipitated and immunoblotted for phospho-ATR (Thr1989).

\section{V.3 Time-dependent reduction of Chk1 phosphorylation occurs in combination treatment}

To investigate whether Wee1 inhibition is directly affecting the activation of ATR-Chk1 signaling, we performed a time-course study. We treated the cells with Wee1 inhibitor in the presence of gemcitabine and harvested them at different time-points after treatment. Western blot analysis showed that inactivation of Chk1 begins after $12 \mathrm{~h}$ of treatment. At 
$24 \mathrm{~h}$ of treatment, the decrease in phospho-Chk1 levels was even stronger. After $8 \mathrm{~h}$ of treatment, we observed an increase in $\mathrm{yH} 2 \mathrm{AX}$ as well as phospho-Chk1 levels due to increased DNA damage response upon combination treatment. However, only after long exposure to the treatment, phosphorylation of Chk1 decreases, suggesting indirect regulation of Wee1 in maintaining Chk1 phosphorylation (Figure V-VI).
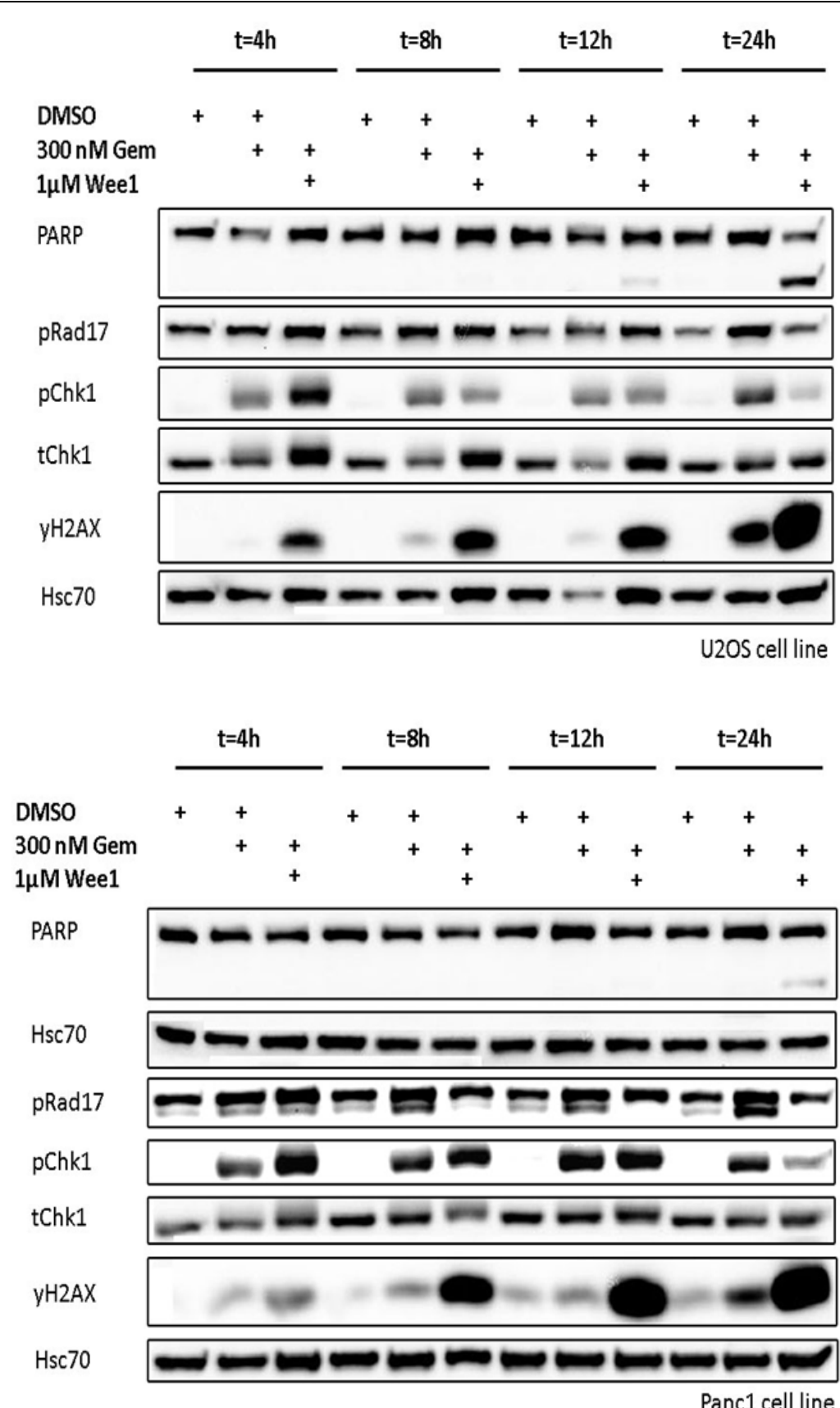

Figure V-VI Chk1 activity reduces in a time-dependent manner upon combining Wee1 inhibition with gemcitabine.

Cells were treated with $1 \mu \mathrm{M}$ Wee1 inhibitor (MK1775) and 300nM gemcitabine. Cells were harvested at different time-points and cell lysate was immunoblotted. tChk1 stands for the total protein while pChk1, pRad17 for phosphorylated forms.Conducted by Yizhu Li. 


\section{V.4 Apoptosis is not the cause of ATR inactivation upon Wee1 inhibition}

We observed an increase in PARP cleavage when Wee1 inhibition was combined with gemcitabine, indicating apoptosis in these cells (Figure V-IV(a)). As apoptosis could lead to dephosphorylation of proteins (Baxter and Lavin 1992) and moreover, PP2A, a phosphatase which can regulate Chk1 dephosphorylation (Leung-Pineda, Ryan, and Piwnica-Worms 2006) is up-regulated during apoptosis (Santoro et al. 1998), we addressed the question whether apoptosis might be a cause for decreased activation of ATR pathway. Therefore, we treated U2OS and Panc1 cells with gemcitabine and/or Wee1 inhibitor in the presence of Z-VAD.fmk, a pan caspase inhibitor that irreversibly binds to catalytic sites of caspase proteases and can inhibit apoptosis (Garcia-Calvo et al. 1998). Analysis of the blots shows that reduction in Chk1 phosphorylation occurs independently of caspase activation (Figure V-VII).
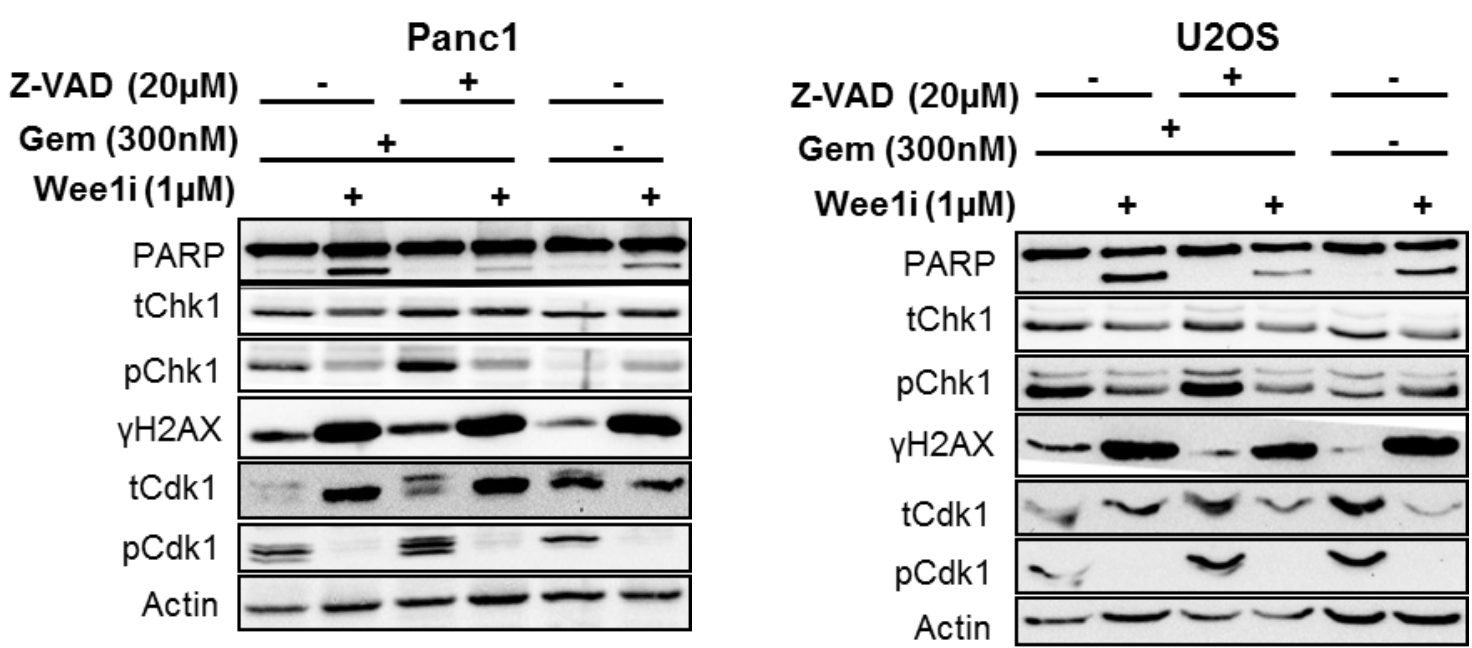

Figure V-VII Caspase activity does not cause loss of Chk1 activation.

Cells were treated with $1 \mu \mathrm{M}$ MK1775 (Wee1i) and 300nM gemcitabine (Gem) in the presence or absence of $20 \mu \mathrm{M}$ caspase inhibitor, Z-VAD.fmk (Z-VAD). After $24 \mathrm{~h}$, the cells were harvested and western blot analysis was done. tChk1, tCdk1 stands for the total proteins while pChk1, pCdk1 for phosphorylated forms.

\section{V.5 Mitotic catastrophe does not lead to down-regulation of ATR- Chk1 pathway}

It is known that Wee1 inhibition in S-phase arrested cells leads to premature mitosis and consequently to mitotic catastrophe (Aarts et al. 2012). As many kinases undergo modulation in their activity while entering into mitosis, our proposition was that ATR phosphorylation changes upon entry of cells into premature mitosis or mitotic catastrophe. 
To address this, we performed western blot analysis in MCF7, a breast cancer cell line. This cell line (with wild-type p53) has been reported to be resistant (to a significant extent) to premature mitosis upon Wee1 inhibition in the presence of gemcitabine than other breast tumor cell lines with mutant p53 (Aarts et al. 2012). We first verified this finding through phospho-H3 staining in flow cytometry. We detected the percentage of cells that stained positive for phospho-H3 with $<4 \mathrm{~N}$ DNA content. This percentage of cells corresponds to the fraction entering into mitosis prematurely. It was found that the amount of cells entering into premature mitosis was indeed significantly less in MCF7 cells as compared to U2OS cells upon combination of Wee1 inhibition with gemcitabine (Figure V-VIII(a)). Afterwards, MCF7 cells were treated with Wee1 inhibitor in the presence/absence of gemcitabine for $24 \mathrm{~h}$ for western blot analysis. We found decreased phosphorylation of Chk1 as well as ATR even in these cells upon Wee1 inhibition with gemcitabine (Figure V-VIII(b,c)). Furthermore, we investigated whether caspase activity affects ATR-Chk1 pathway in MCF7 cells. For the same, we performed western blotting with combination treatment in the presence of Z-VAD.fmk and could not rescue inactivation of Chk1 (Figure V-VIII(b)).

From here, we infer that premature mitosis or mitotic catastrophe is not necessary for the deactivation of the ATR-Chk1 pathway.

(a)
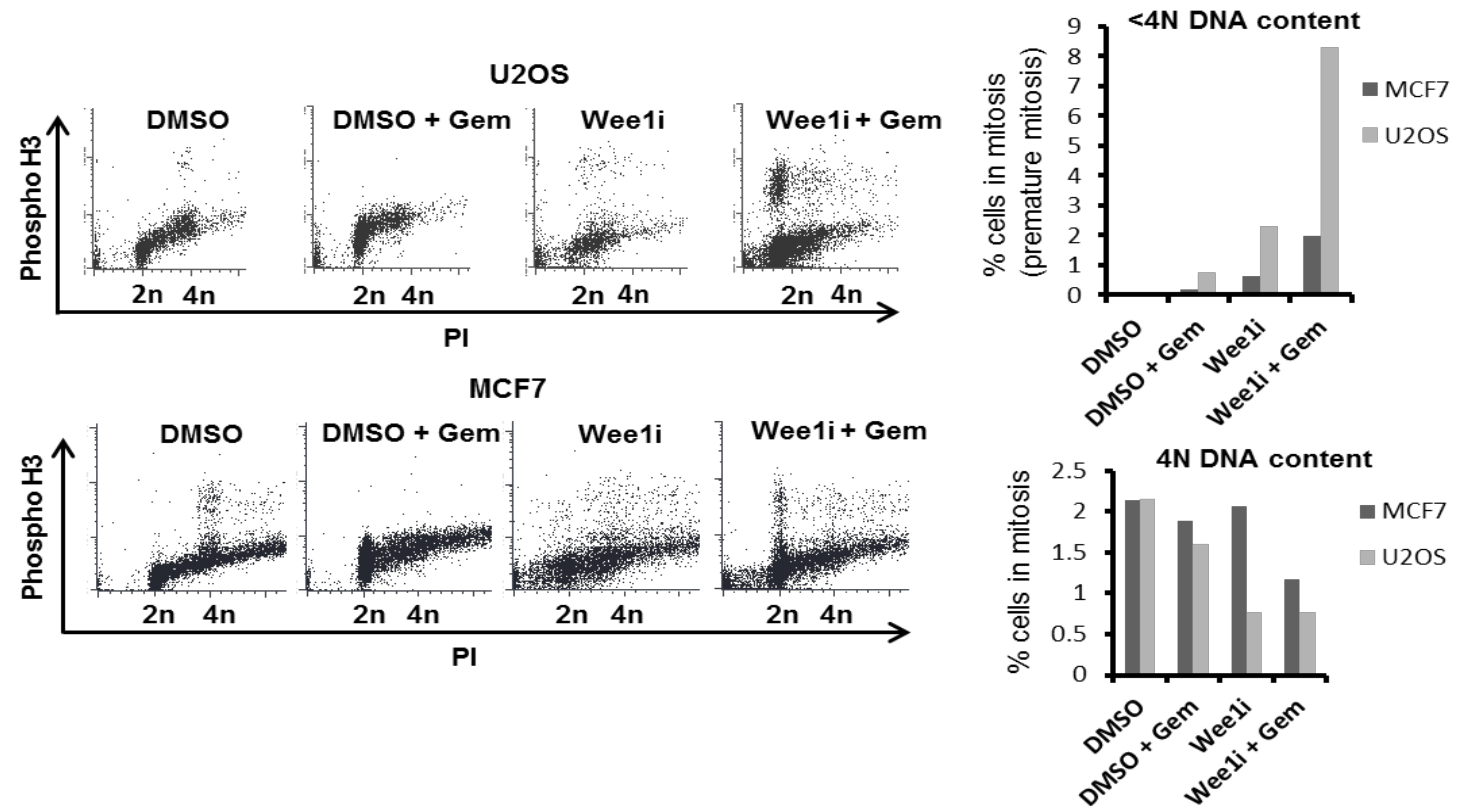

(b) 


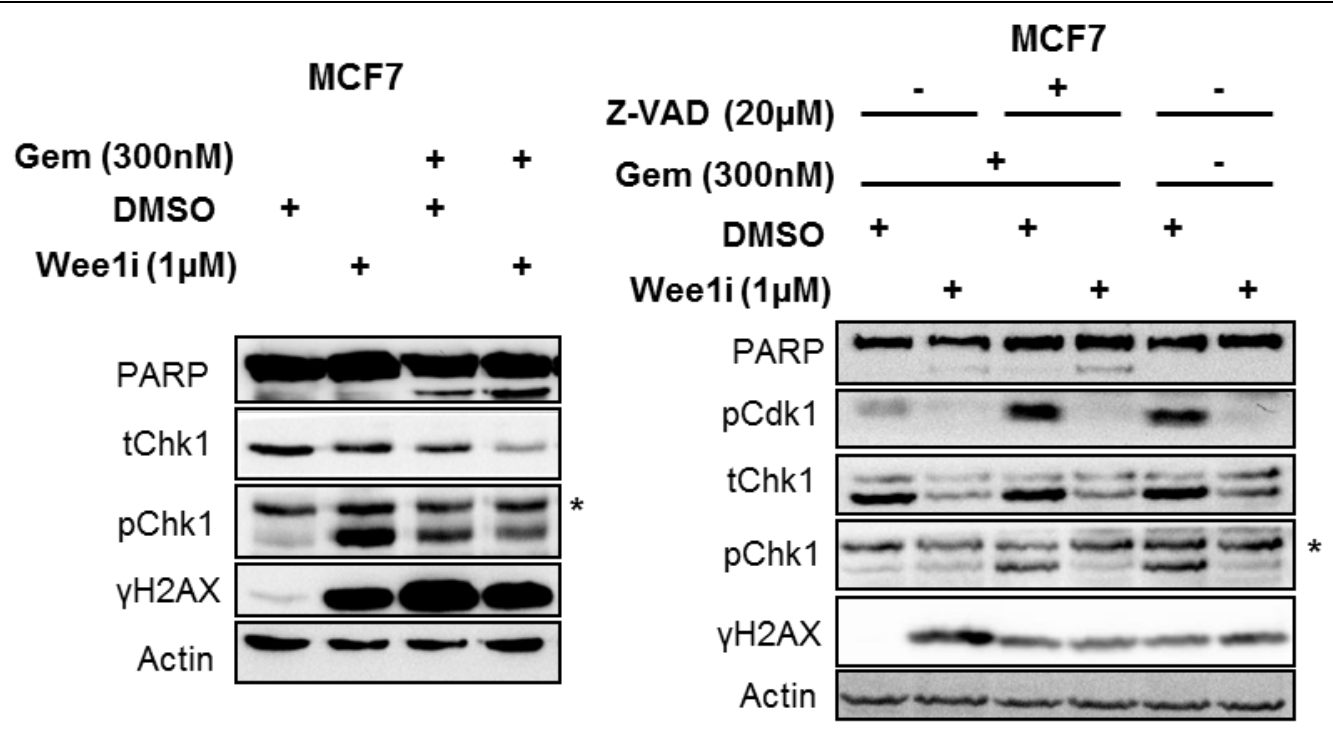

(c)

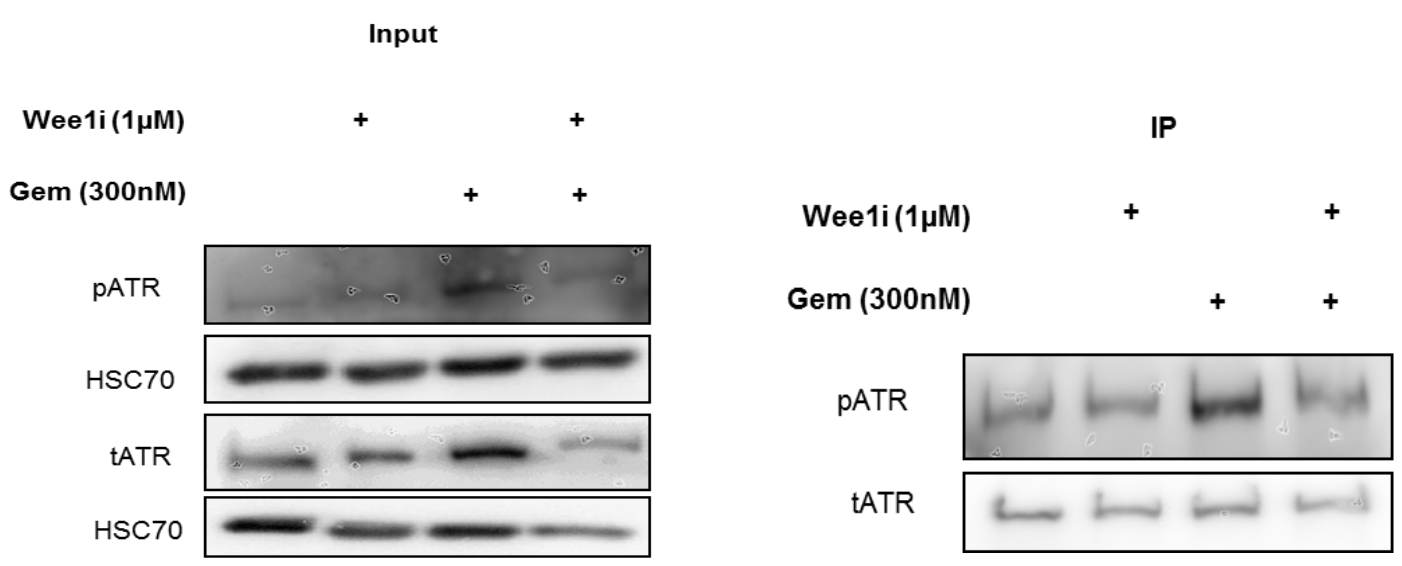

Figure V-VIII Mitotic catastrophe does not lead to reduction in ATR-Chk1 activity.

(a) U2OS and MCF7 cells were treated with $1 \mu \mathrm{M}$ Wee1 inhibitor/DMSO in the presence or absence of $300 \mathrm{nM}$ gemcitabine for $24 \mathrm{~h}$. Cells were harvested, stained with $\mathrm{PI}$ and phospho-H3. It was followed by flow cytometric analysis. (b) MCF7 cells were treated as mentioned in (a) either in the presence or absence of Z-VAD.fmk and cell lysate was immunoblotted. tChk1 stands for the total protein while pChk1 for phosphorylated forms. * represents unspecific band. (c) MCF7 cells were treated as in (a) and then cells were harvested and cell lysate was prepared, ATR was immunoprecipitated and immunoblotted for phospho-ATR (Thr1989).

\section{V.6 Decreased activation of ATR-Chk1 pathway is mediated through Cyclin-dependent kinases}

\section{V.6.1 Inhibition of Cyclin-dependent kinases (Cdks) using roscovitine restores Chk1 phosphorylation}

Wee1 is a checkpoint regulator that has a major role in controlling the transition of cells through the S- and G2/M-phases of the cell cycle; it directly phosphorylates and inhibits 
Cdk1 and Cdk2 at the conserved Tyr15 residue (Guertin et al. 2012). Thus, Wee1 inhibition can lead to Cdk1/2 activation. To test whether the inhibition of the ATR-Chk1 pathway by Wee1 inhibition is due to Cdk activation, we performed inhibition of Cdks using roscovitine along with Wee1 inhibition and gemcitabine. Roscovitine is a potent, reversible and selective inhibitor of Cdks and binds competitively to the ATP-binding domain of these kinases (Meijer et al. 1997). Western blot analysis showed rescue of Chk1 and ATR phosphorylation when Cdks were inhibited (Figure V-IX(a, b)).

These findings imply that inactivation of the ATR-Chk1 pathway is mediated through Cdks upon Wee1 inhibition.

(a)

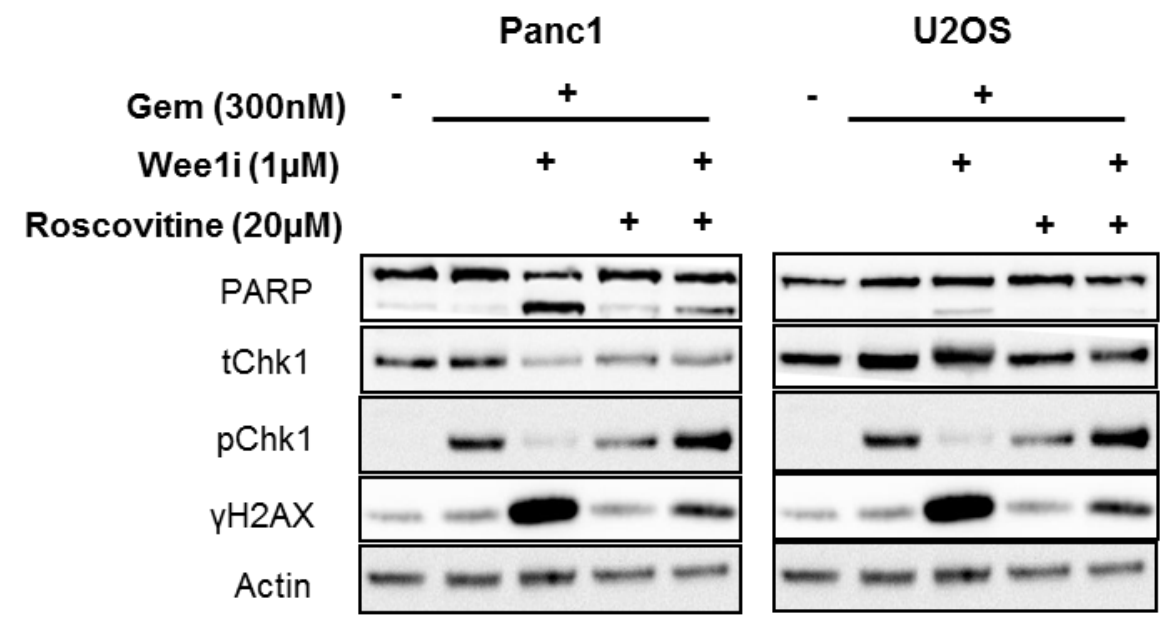

(b)

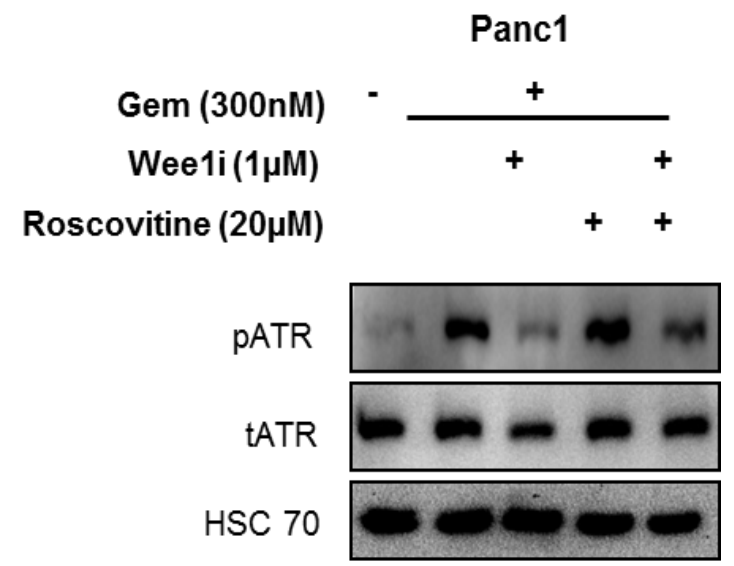

Figure V-IX Cdk inhibition rescues decreased Chk1 and ATR activity upon Wee1 inhibition with gemcitabine.

(a) A potent and selective inhibitor of Cdks, roscovitine, restores Chk1 phosphorylation. Panc1 and U2OS cells were treated with $20 \mu \mathrm{M}$ roscovitine in the presence or absence of Wee1i and Gem, after $24 \mathrm{~h}$ cells were harvested and western blot analysis was performed. (b) Cells were treated as in (a), harvested and cell lysate was stained for phospho-ATR and total ATR. 


\section{V.6.2 Inhibition of Cdk1 could recover Chk1 phosphorylation}

We also used a selective inhibitor of Cdk1, RO-3306. This inhibitor is an ATP-competitive inhibitor of Cdk1 and has nearly 10-fold selectivity relative to Cdk2 (Vassilev et al. 2006). We found that this inhibitor, when combined with Wee1 inhibition and gemcitabine, could restore the phosphorylation of Chk1 (Figure $\mathbf{V - X}$ ). In conclusion, Cdk1 plays an active role in inactivating ATR-Chk1 pathway.

However, there are no studies showing direct involvement of Cdk in ATR inactivation. Therefore, we tested some of the known substrates of Cdk which could potentially mediate ATR pathway inactivation.

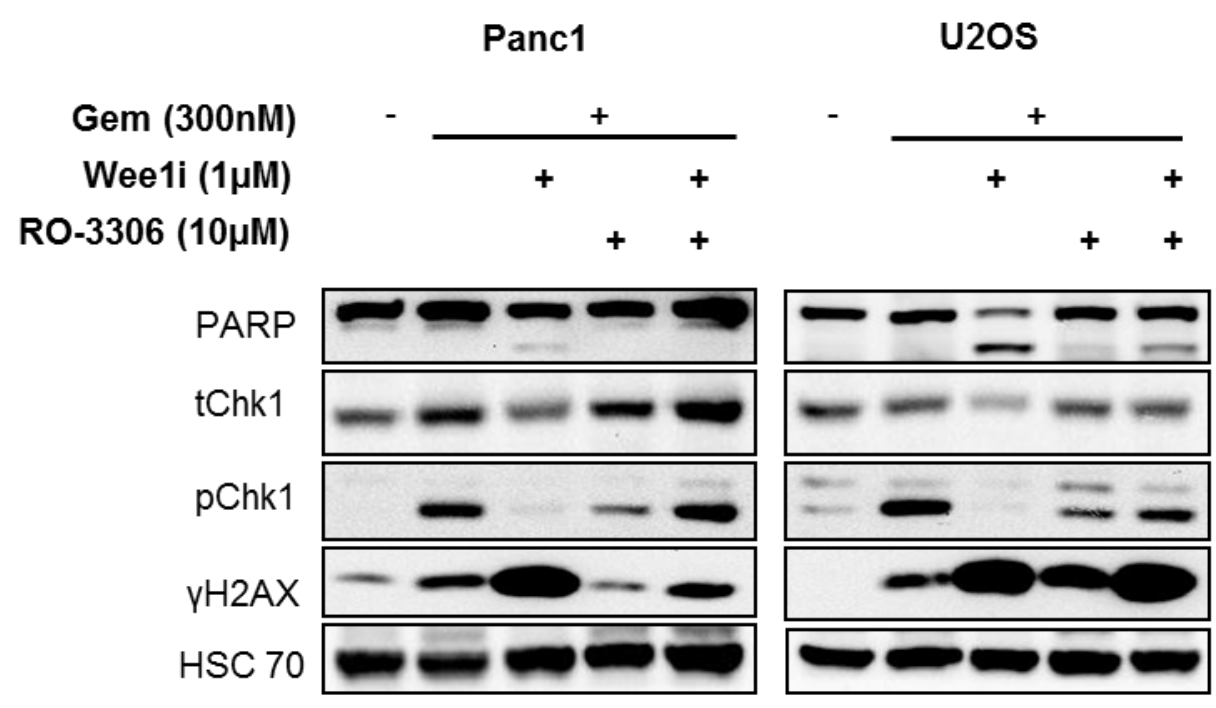

Figure V-X Cdk1 inhibition recovers Chk1 and Rad17 phosphorylation upon Wee1 inhibition and gemcitabine treatment.

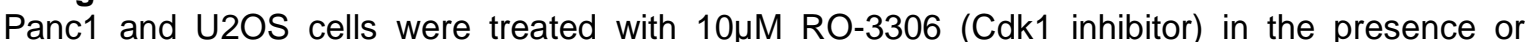
absence of Wee1i and Gem, after $24 \mathrm{~h}$ cells were harvested and western blot analysis was performed.

\section{V.7 Cdk substrates Mus81 and Retinoblastoma protein do not mediate down-regulation of ATR pathway}

\section{V.7.1 Mus81 does not govern inactivation of ATR signaling pathway}

Mus81 is a structure-specific endonuclease involved in cleaving branched DNA (Osman and Whitby 2007). It forms a heterodimeric complex with Eme1 (another endonuclease) which is found to be controlled by Wee1 directly or through Cdk (Domínguez-Kelly et al. 2011). Wee1 inhibition hyperactivates Cdk, which then leads to deregulation of DNA replication; subsequently, aberrant DNA structures are formed which are substrate of 
Mus81/Eme1 (Martín, Domínguez-Kelly, and Freire 2011). Upon inhibition of Wee1, Mus81 processes DNA breaks and ensures recovery from replication stress (Hanada et al. 2007, Murfuni et al. 2013). We hypothesized that Mus81 might be regulating the ATR pathway so that cells could move on in the cell cycle once DNA breaks are processed. To test this, we depleted the cells of Mus81 and Wee1 and treated them with gemcitabine for $24 \mathrm{~h}$. Western blotting analysis revealed that co-depletion of Wee1 and Mus81 could not rescue decreased phosphorylation of Chk1 and Rad17, compared to Wee1 single knockdown. However, we found that knockdown of Mus81, in combination with gemcitabine, leads to decreased $\mathrm{\gamma H} 2 \mathrm{AX}$ and Chk1 phosphorylation (Figure V-XI). We do not fully understand this phenomenon but speculate that processing of stalled replication forks by Mus81-Eme1 complex might lead to the generation of a DNA damage response. In line with our speculation, Domínguez-Kelly and colleagues observed a diminished DNA damage response, generated by Wee1 removal, upon co-depletion of Mus81 (Domínguez-Kelly et al. 2011).

Thus, these results illustrate that Mus81 does not lead to inactivation of the ATR pathway upon inhibition of Wee1 in the presence of gemcitabine. Instead, Mus81 maintains Chk1 activation in the presence of stalled replication forks caused by gemcitabine.

(a)

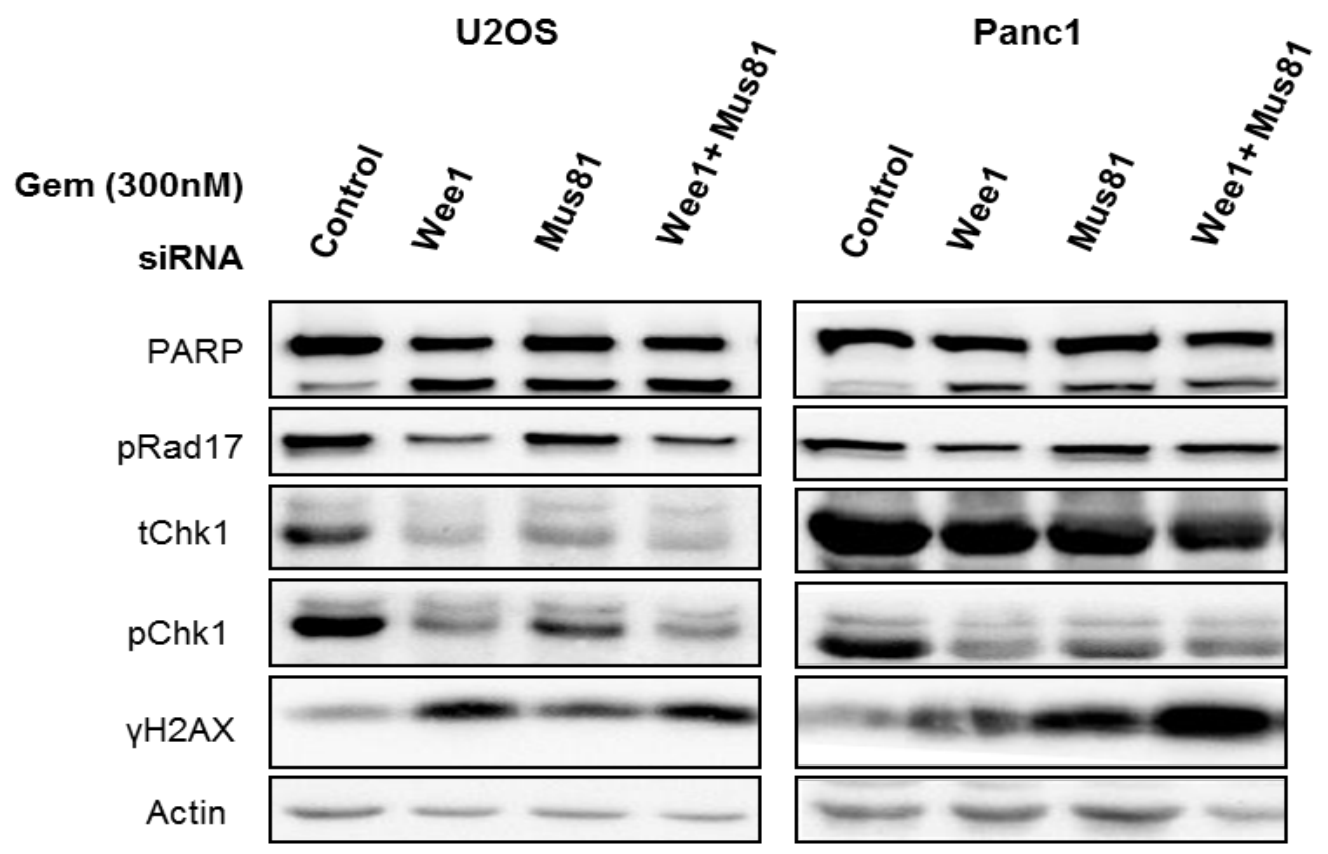

(b) 


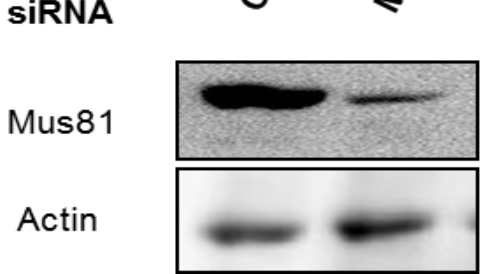

Figure V-XI Mus81 does not mediate ATR-Chk1 inactivation upon Wee1 inhibition and gemcitabine treatment.

(a) Cells were made deficient of Wee1 and/or Mus81 by transfecting with siRNAs (Wee1\#1, Mus81) for $48 \mathrm{~h}$, followed by gemcitabine treatment for $24 \mathrm{~h}$. Cells were harvested and western blot analysis was performed. Negative Control No.1 siRNA transfected cells were used as control (Control). (b) Panc1 cells were transfected with negative control no.1 and Mus81 \#1 siRNA. After $48 \mathrm{~h}$, cells were harvested and protein lysate was immunostained.

\section{V.7.2 The Retinoblastoma protein, negatively regulated by Cdks, does not affect the ATR pathway}

Functional inactivation of the Retinoblastoma protein (also referred to as $\mathrm{pRb}$ ) has been found to be controlled by three distinct Cyclin-Cdk complexes, namely CyclinD-Cdk4/6, CyclinE-Cdk2 and CyclinA-Cdk2/1 (Lundberg and Weinberg 1998). As Cdks could negatively regulate $\mathrm{pRb}$, we hypothesized that $\mathrm{pRb}$ might be involved in maintaining the activation of ATR signaling pathway. pRb, being a repressor of the E2F transcription factor family could regulate ATR, e.g. through E2F-mediated transcription.

To assess whether there is a change in the levels of ATR mRNA, we performed quantitative RT-PCR for ATR in Panc1 cells where Wee1 was knocked down in the presence of gemcitabine. It was found that the mRNA levels of ATR did not significantly change upon knockdown of Wee1 (Figure V-XII(a)). This observation argues against the control of ATR at the level of transcription.

To test whether $p R b$ itself could affect the activation of ATR, we used Hela cells that contain inactive $\mathrm{pRb}$ due to expression of the E7 protein from papilloma virus, which can bind and inactivate pRb (Gonzalez et al. 2001). We treated this cell line with Cdk1 inhibitor in the presence of Wee1 inhibitor and gemcitabine and analyzed the proteins through western blotting. We observed that even in Hela cells, Cdk inhibition could rescue the phosphorylation of Chk1 as well as Rad17 (Figure V-XII(b)). This experiment shows that the Retinoblastoma protein does not sustain the ATR pathway and that Wee1 inhibition interferes with ATR-Chk1 activity independently of $p R b$. 
(a)

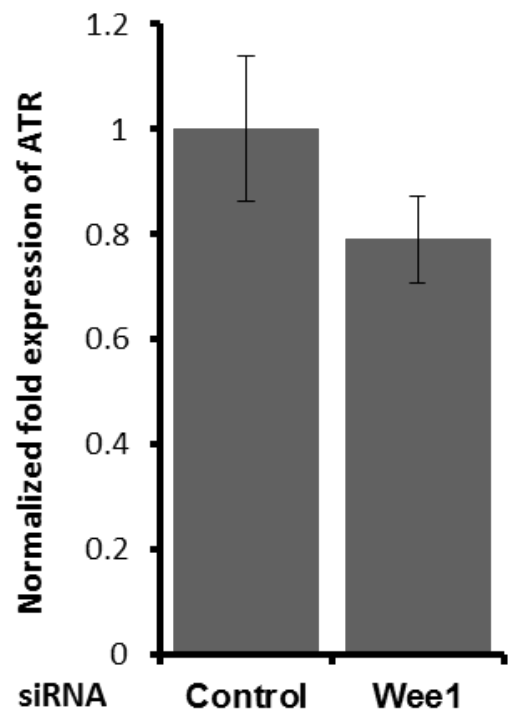

(b)

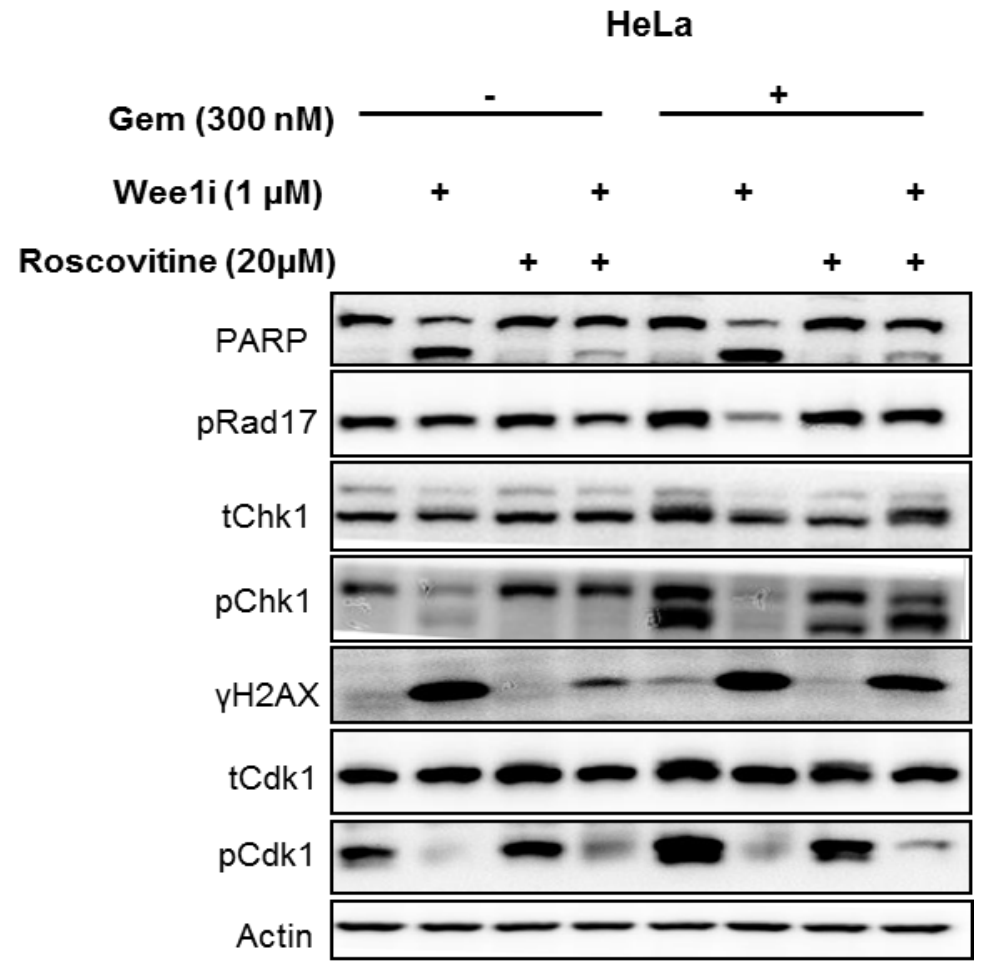

Figure V-XII Retinoblastoma protein does not mediate the down-regulation of ATR-Chk1 activity upon inhibition of Wee1 and gemcitabine treatment.

(a) Panc1 cells were transfected with 10nM siRNA against negative control no. 1 (control) or Wee1\#1 (Wee1). After 48 h, cells were treated with gemcitabine for $24 \mathrm{~h}$. Cells were harvested, RNA was isolated and quantitative RT-PCR was performed. 36B4 mRNA was used as a reference gene for normalization. Error bars represent standard error of the mean. CFX manager software was used for the calculations. (b) HeLa cells were treated with Wee1i, gemcitabine and/or roscovitine for $24 \mathrm{~h}$. Cells were harvested and immunoblot analysis was done. 


\section{V.8 Polo- like kinase 1 (Plk1) impedes the ATR- Chk1 pathway}

\section{V.8.1 Inhibition of PIk1 recovers decreased Chk1 and Rad17 activation upon Wee1 inhibition and gemcitabine}

It has been shown that the yeast homolog of Plk1, cdc5, is activated by the Cdk1, homolog cdc28 in yeast (Mortensen et al. 2005) (Simpson-Lavy and Brandeis 2011). Plk1 is also known to down-regulate the ATR-Chk1 pathway by acting at different levels of this signaling pathway. It phosphorylates the adaptor protein Claspin and marks it for degradation by the ubiquitin ligase $\mathrm{SCF} \beta-\operatorname{TrCP} 1 / 2$, thereby restraining Chk1 activation and regulating the recovery from the DNA replication checkpoint response (Niels Mailand et al. 2006), (Peschiaroli et al. 2006). Another level of regulation of ATR-Chk1 signaling pathway by Plk1 is through Sae2 in yeast, the functional ortholog of human CtIP (Donnianni et al. 2010); CtIP is involved in DNA resection and is required to sustain checkpoint signaling (Kousholt et al. 2012). To investigate the role of Plk1 in the negative regulation of ATR-Chk1 activity, we incubated cells with a PIk1 inhibitor, in the presence of the Wee1 inhibitor and gemcitabine. Through immunoblot analysis, it was found that the inhibition of Plk1 could recover the decreased phosphorylation of Chk1. Hence, Plk1 activity is required for the attenuation of ATR-Chk1 signaling upon Wee1 inhibition (Figure V-XIII(a)).

Plk1 has a phospho-peptide binding domain that binds to the proteins 'primed' or phosphorylated by kinases, thereby facilitating localization of Plk1. Kinases known to prime Plk1 substrates include Cdk1, that drives the cells into mitosis (K. S. Lee et al. 2008). Plk1 activity is also regulated by Aurora A kinase through an auxiliary protein, Bora (Seki et al. 2008). Binding of Bora to Plk1 facilitates the phosphorylation of Plk1 at Thr210 by Aurora A causing the activation of Plk1. To validate the activation of Plk1 upon Wee1 inhibition and its inactivation upon Plk1 and Cdks inhibition, we performed western blot analysis after treatment with these inhibitors. We found that the levels of phosphorylated Plk1 (Thr210) increased with Wee1 inhibition and that this phosphorylation is decreased when Plk1 and Cdk inhibitors were added (Figure V-XIII(b)). Therefore, we conclude that Plk1 activity is increased upon Wee1 inhibition with gemcitabine. 
(a)

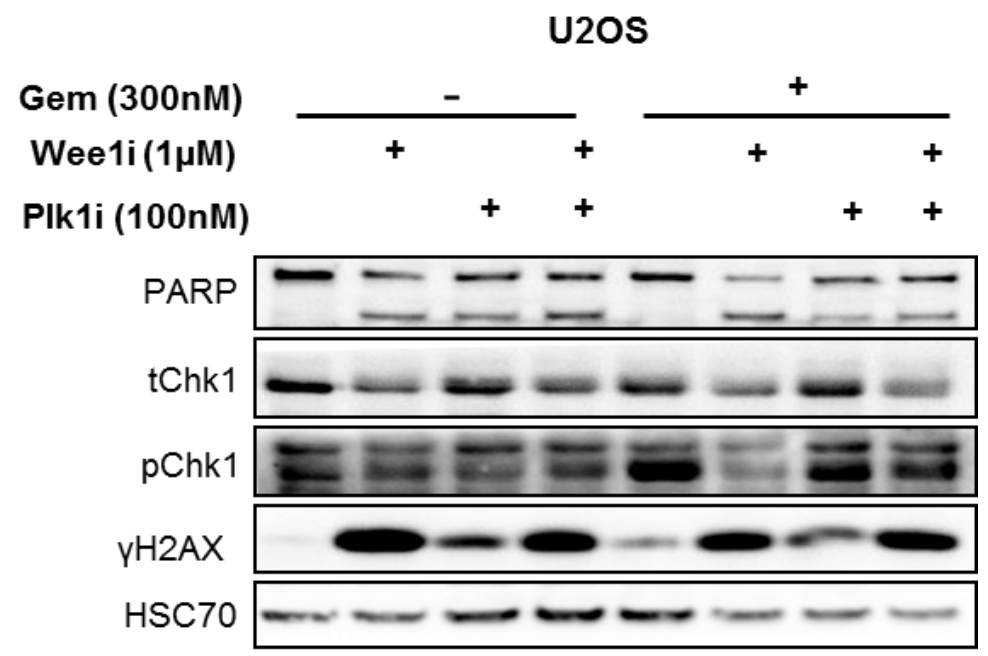

Panc1

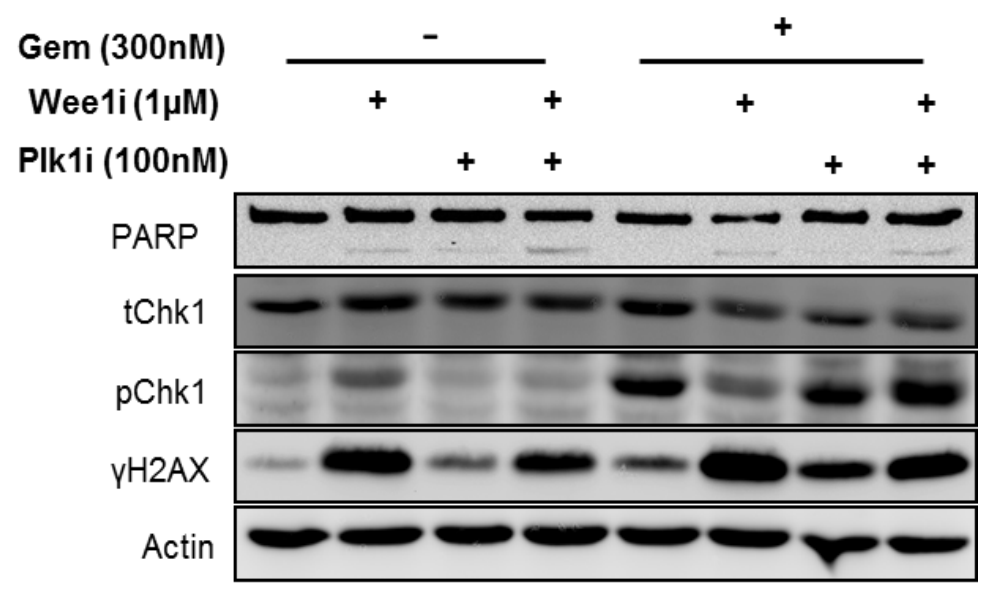

(b)

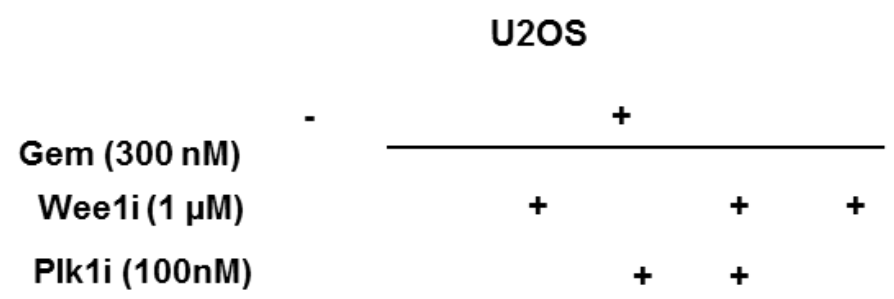

Roscovitine $(20 \mu \mathrm{M})$

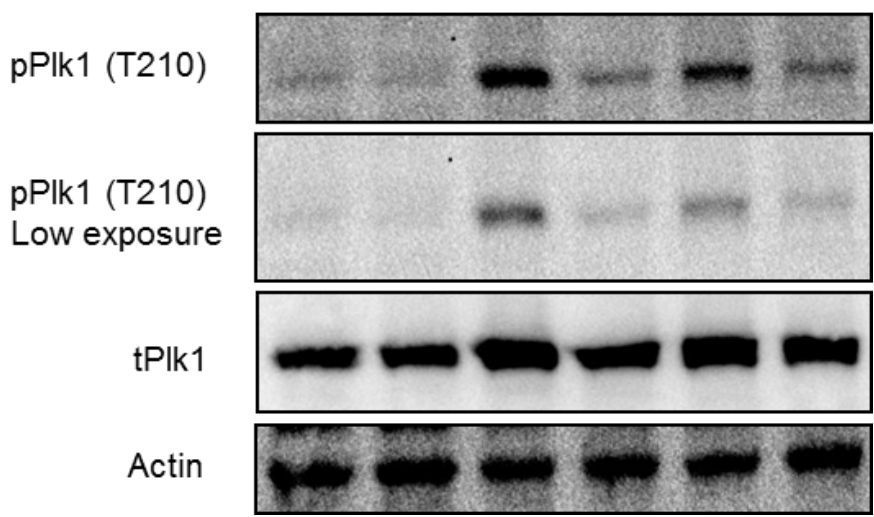




\section{Panc1}

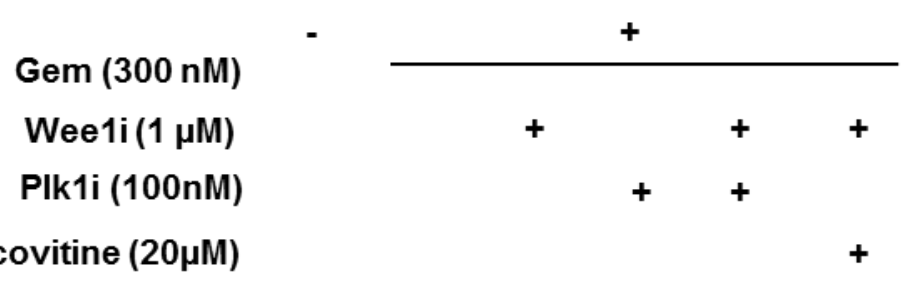

Roscovitine $(20 \mu \mathrm{M})$

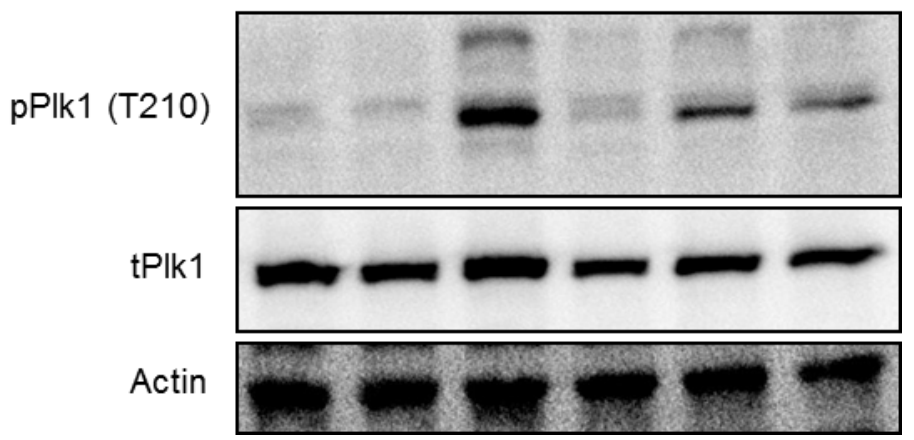

Figure V-XIII Inhibition of PIk1 rescues ATR-Chk1 activity.

(a) Panc1 and U2OS cells were treated with Wee1i or DMSO with or without gemcitabine in the presence or absence of Plk1 inhibitor, GSK 461364 (referred to as Plk1i) at the concentrations indicated in figure for $24 \mathrm{~h}$. Cells were harvested and cell lysate was immunoblotted. Blots were stained for phosphorylation of ATR substrates- Chk1 and Rad17. HSC 70 or $\beta$-Actin was stained as loading control. (b) Panc1 and U2OS cells were treated with Wee1i, Plk1i and combination of Wee1i with Plk1i or Roscovitine in the presence of gemcitabine at the indicated concentrations for $8 \mathrm{~h}$. Cells were harvested and cell lysate was immunoblotted. Blots were stained for phosphorylation of Plk1 (Thr210). $\beta$-Actin was stained as loading control. DMSO treated cells were used as negative control.

Immunoblot in (a) for Panc1 cells was conducted by Indira Memet.

\section{V.8.2 Plk1 mediates inactivation of Chk1 through Claspin degradation}

Claspin is an adaptor protein which binds to Chk1 and facilitates its activation by ATR (Chini and Chen 2003). To determine whether Chk1 inactivation by PIk1 is mediated through Claspin, we checked the levels of Claspin upon Wee1 inhibition as well as Plk1 and Cdks inhibition. Through immunoblotting, we showed that Claspin level was decreased upon Wee1 inhibition with gemcitabine and that it was restored when Plk1 or Cdks inhibitor is combined (Figure V-XIV(a)). We could also show by western blotting that removal of Claspin reduces Chk1 phosphorylation (Figure V-XIV(b,c)). From here, we deduce that degradation of Claspin by activated Plk1 hampers Chk1 activity. 


\section{Results}

(a)

U2OS

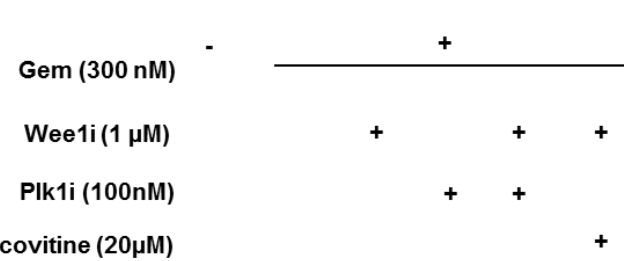

Panc1

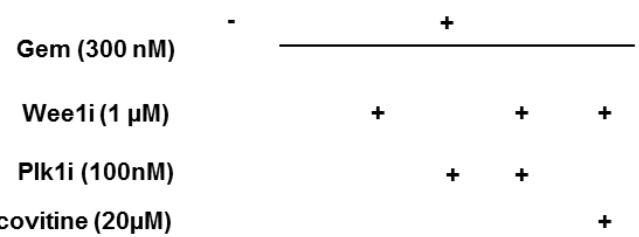

Roscovitine $(20 \mu \mathrm{M})$

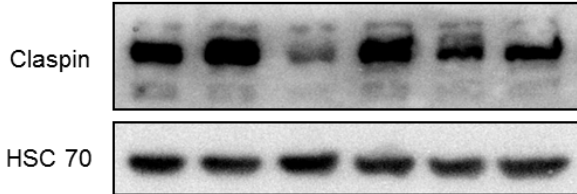

(b)

U2OS

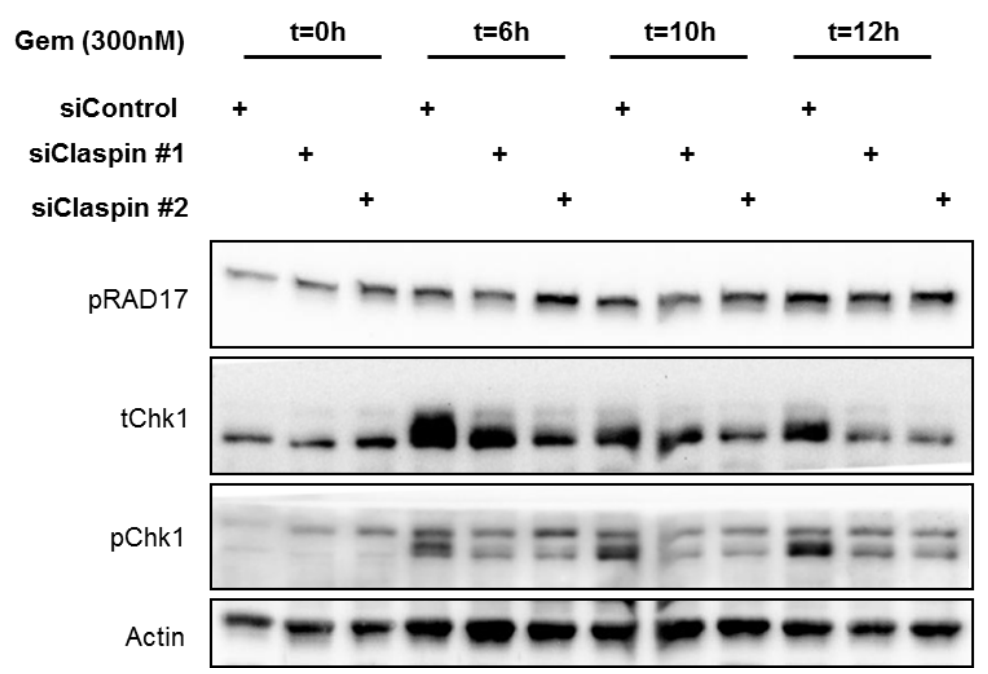


Panc1

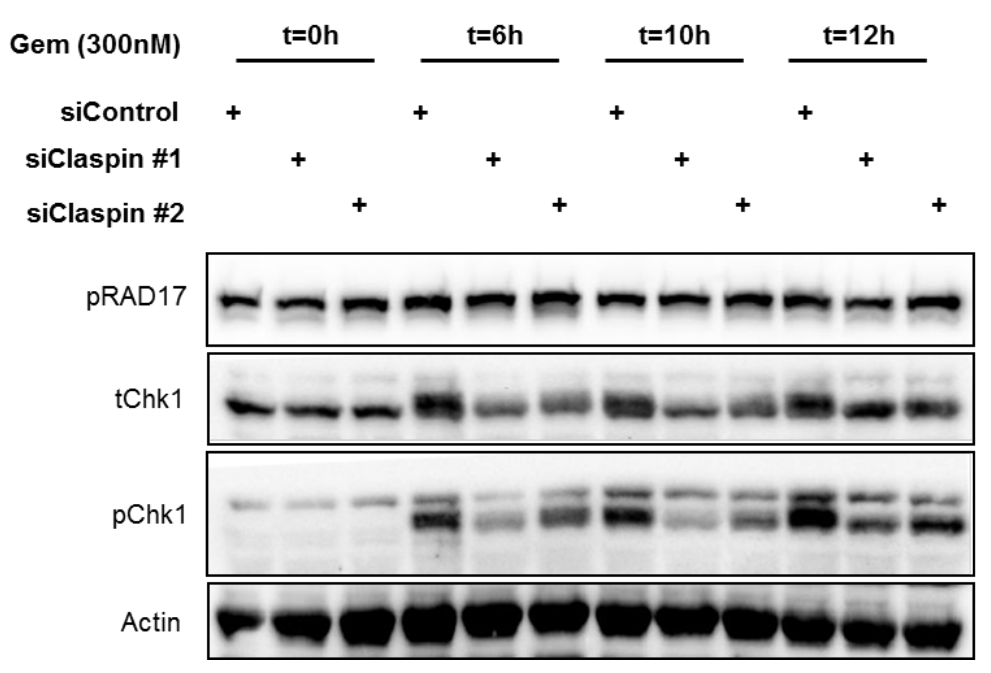

(c)

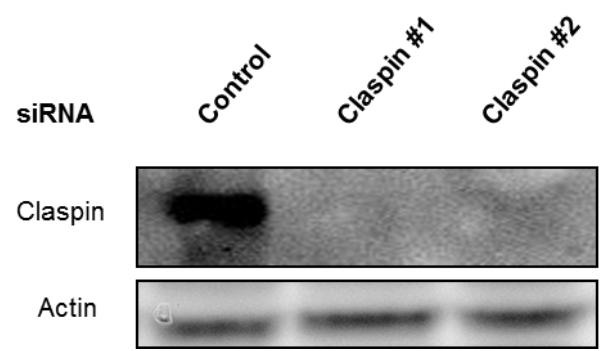

Figure V-XIV Plk1 causes Claspin degradation which leads to Chk1 inactivation upon Wee1 inhibition with gemcitabine.

(a) Panc1 and U2OS cells were treated with Wee1i, Plk1i and combination of Wee1i with Plk1i or Roscovitine in the presence of gemcitabine at the indicated concentrations for $8 \mathrm{~h}$. Cells were harvested and cell lysate was immunoblotted. Blots were stained for total levels of Claspin. HSC 70 was stained as loading control. DMSO treated cells were used as negative control. (b) Claspin was knocked down in the cells by transfecting with 10nM siRNAs for $48 \mathrm{~h}$, followed by treatment with 300nM gemcitabine. Cells were harvested at different time points after gemcitabine addition i.e., $0 \mathrm{~h}, 6 \mathrm{~h}, 10 \mathrm{~h}$ and $12 \mathrm{~h}$. Cell lysate was immunoblotted and stained for Chk1 and Rad17 phosphorylation. $\beta$-Actin was used as loading control. (c) Immunoblot showing the Claspin knockdown using two different siRNAs in Panc1 cells. 


\section{Clinical relevance of combination therapy}

\section{V.9 Nutlin-3 pretreatment attenuates DNA damage response and apoptosis upon Wee1 inhibition with gemcitabine in p53- proficient cells}

\section{V.9.1 U2OS, a cell line with wild-type p53, resists cytotoxic effects of combination treatment upon nutlin-3 pretreatment}

Nutlin-3 is an inhibitor of Mdm2-p53 interaction, thereby, causing non-genotoxic accumulation of p53 which results in cell cycle arrest and/or apoptotic response (Miyachi et al. 2009). In a previous study in our lab, it has been shown that the pretreatment of U2OS cells with nutlin-3 followed by transient exposure to nutlin-3 and gemcitabine reduces gemcitabine related- cytotoxicity in these cells (Kranz and Dobbelstein 2006). We tested whether treatment of U2OS cells with nutlin-3 prior to combination therapy, Wee1 inhibitor and gemcitabine, could decrease the cytotoxic effects of the latter treatment. To address this, we treated the cells with sub-lethal dose of nutlin- 3 for $24 \mathrm{~h}$, followed by addition of nutlin-3 with Wee1 inhibitor in the presence of gemcitabine for another $24 \mathrm{~h}$. Western blot analysis showed that $\mathrm{yH} 2 \mathrm{AX}$ levels decrease upon nutlin pre-treatment, apart from it, cleaved PARP, cleaved caspase-3 and phosho-H3 levels also decreases as compared to nutlin untreated cells. Also, p53 was stabilized and there was induction of p21 with nutlin treatment reflecting the activity of nutlin (Figure V-XV). From here, we deduce that treatment of U2OS cells with nutlin prior to combination therapy reduces DNA damage response, apoptosis as well as the number of cells entering into mitosis caused by combination therapy alone. 

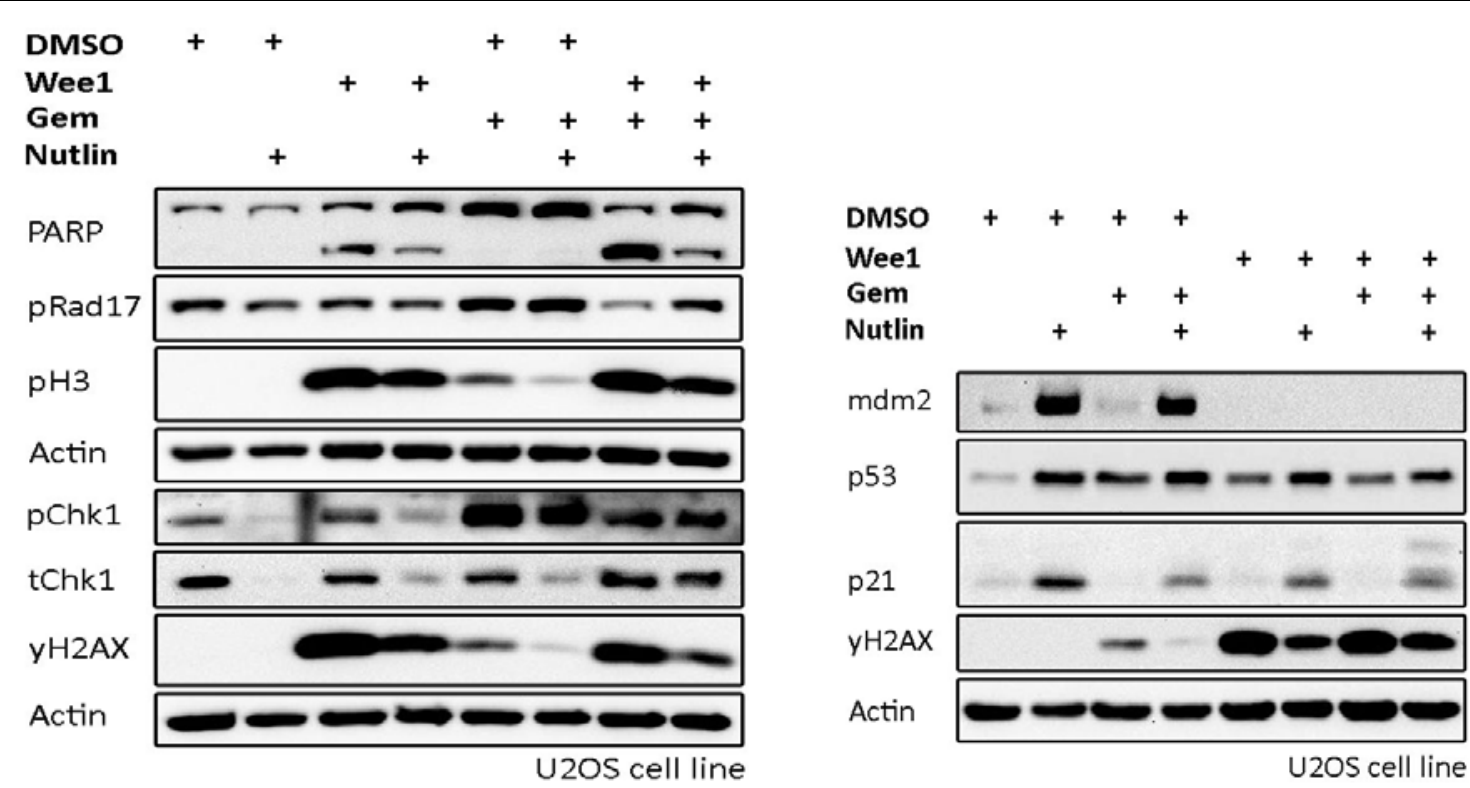

Figure V-XV Nutlin-3 attenuates the cytotoxicity caused by combination of Wee1 inhibitor and gemcitabine.

U2OS cells were treated with $8 \mu \mathrm{M}$ nutlin- 3 for $24 \mathrm{~h}$, followed by treatment with $1 \mu \mathrm{M}$ Wee 1 inhibitor, $300 \mathrm{nM}$ gemcitabine and $8 \mu \mathrm{M}$ nutlin-3 for another $24 \mathrm{~h}$. Cells were harvested and immunoblot analysis was performed. Conducted by Yizhu Li.

\section{V.9.2 Wild-type p53 is required for protective effects of nutlin-3}

To determine whether p53 is necessary for the protective effects of nutlin-3 against combination therapy, we performed western blot analysis in an isogenic pair of cell lines, derived from HCT116, a human colon carcinoma cell line. We treated both the cell lines, HCT116wtp53 and HCT116p53-/-, in the similar manner as for aforementioned U2OS cells. HCT116 cells with wild-type p53 (HCT116wtp53) showed similar resistance to cytotoxic effects of combination therapy upon nutlin-3 pretreatment. In contrast, HCT116 lacking p53 (HCT116p53 ${ }^{-/}$) did not show any effect of nutlin-3 (Figure V-XVI).

This shows that p53 is essential for the protective effect of nutlin-3 against adverse effects of combination therapy. 


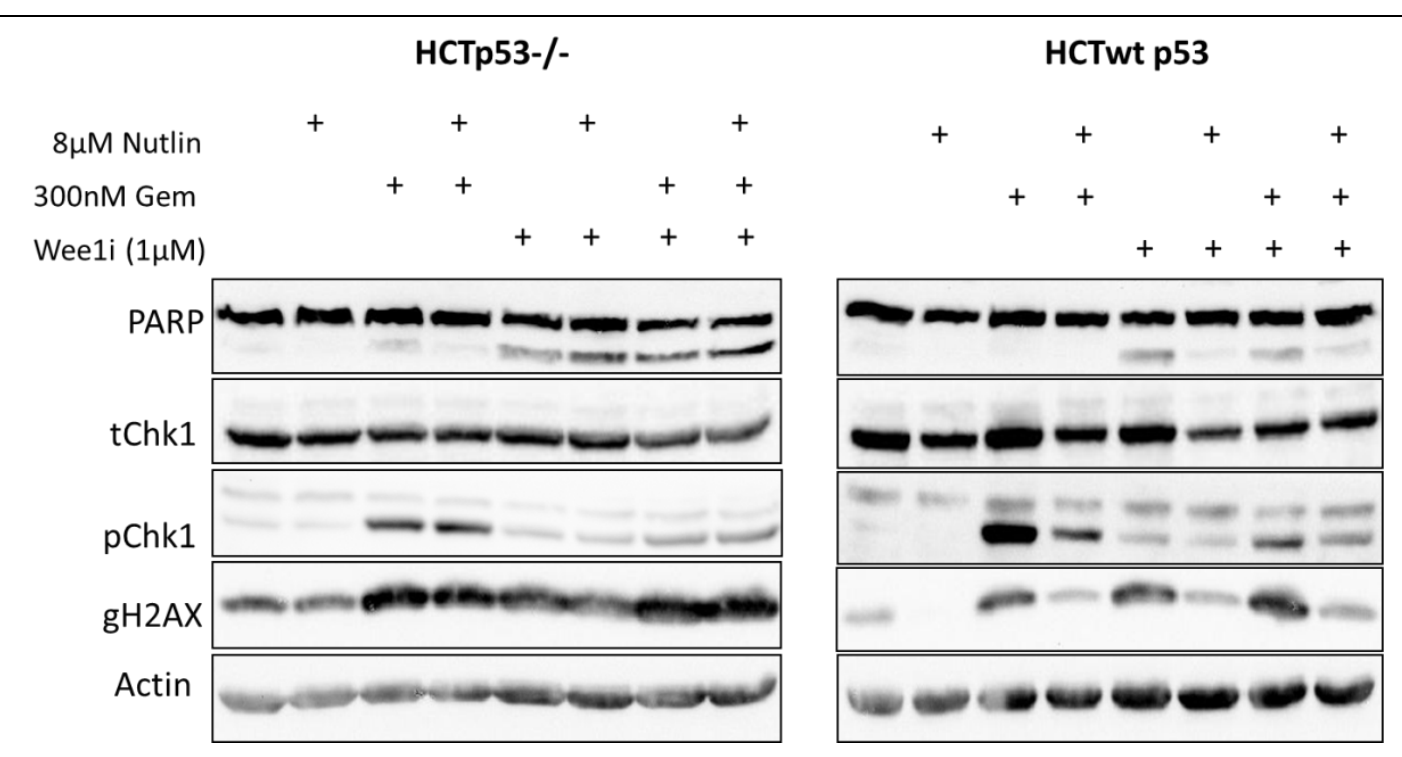

Figure V-XVI p53 is required for protection by nutlin-3.

Isogenic pair of HCT116 cells was pretreated with nutlin-3 for $24 \mathrm{~h}$, followed by treatment with Wee1 inhibitor, gemcitabine and nutlin-3 for another $24 \mathrm{~h}$. Cells were harvested and immunoblotted.

\section{V.10 Long-term survival of cells treated with Wee1 inhibitor and gemcitabine increases upon pre-treatment with nutlin-3}

To assess the long-term survivability of cells when pretreated with nutlin-3, we treated U2OS cells with nutlin for $24 \mathrm{~h}$ followed by exposure to Wee1 inhibitor in the presence or absence of gemcitabine and nutlin-3. Confluency was measured and plotted corresponding to Day 1. After $24 \mathrm{~h}$, all chemicals were washed away, fresh medium was added and confluency of cells was measured using Celigo cytometer subsequently each day for 12 days. We observed that nutlin-3 increases the survival of cells treated with Wee1 inhibitor and gemcitabine as compared to nutlin untreated cells (Figure V-XVII).

From these observations, we can say that normal proliferating cells with wild- type p53 can resist the adverse effects of combination therapy by addition of nutlin while cancer cells with mutant p53, ineffective towards nutlin-3, would die. 


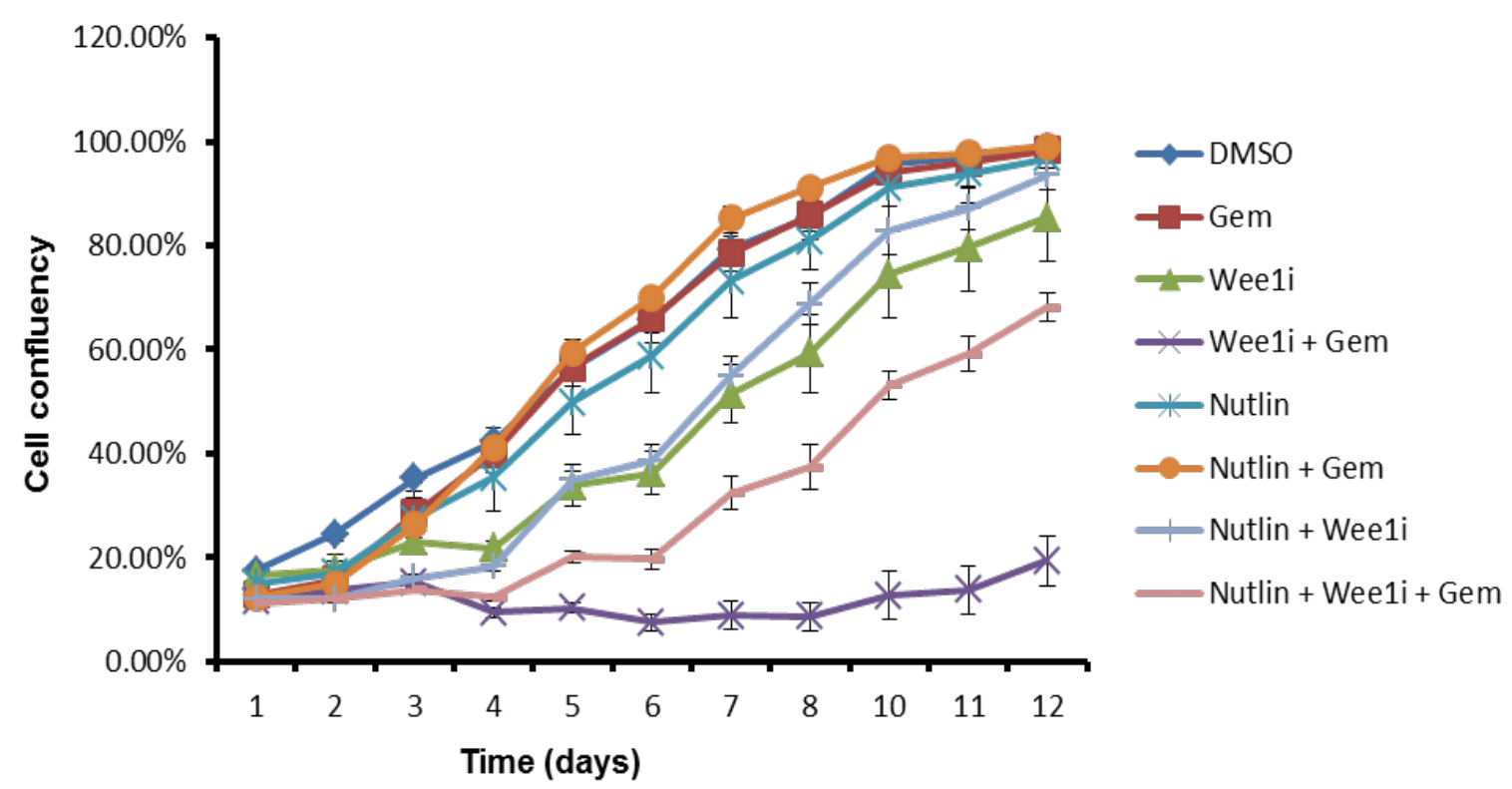

Figure V-XVII Pretreatment with nutlin-3 increases long-term survival of the cells treated with Wee1 inhibitor and gemcitabine.

U2OS cells were treated with $4 \mu \mathrm{M}$ nutlin- 3 for $24 \mathrm{~h}$, followed by treatment with $0.5 \mu \mathrm{M}$ Wee1i and $5 \mathrm{nM}$ gemcitabine with $4 \mu \mathrm{M}$ nutlin-3. After $24 \mathrm{~h}$, all drugs were removed and fresh medium was added. Cells were incubated for 12 days and confluency was measured each day using brightfield microscopy (Celigo cell cytometer). Error bars represent the SD, n=3. Conducted by Yizhu Li.

\section{V.11 Addition of nutlin-3 protects cells from premature mitosis resulting from combination therapy}

Combination of Wee1 inhibition with gemcitabine leads to premature mitosis and consequently to mitotic catastrophe. We investigated whether addition of nutlin could reduce the amount of cells entering into mitosis prematurely, which might be a contributing factor towards the protective effects of nutlin-3 pretreatment. Therefore, we performed flow cytometry with the cells treated with combination therapy in the presence as well as absence of nutlin and stained them with phospho-H3 antibody and propidium iodide. We treated the cells with combination therapy for $8 \mathrm{~h}$ and detected the percentage of cells that stained positive for phospho-H3 with $<4 \mathrm{~N}$ DNA content. This percentage of cells corresponds to the fraction entering into mitosis prematurely. It was observed that the fraction of the cells entering into mitosis prematurely were significantly less upon nutlin-3 pretreatment after 8h of combination treatment (Figure V-XVIII). We chose $8 \mathrm{~h}$ time-point for the treatment as after $8 \mathrm{~h}$, there were a high number of cells entering into premature mitosis. (Refer to Figure VIII-IV in appendix)

We deduce from this study that nutlin pretreatment can reduce the number of cells entering premature mitosis. Thus, nutlin or its derivatives may turn out to be useful in the clinics for protection of normal proliferating tissues. 
(a)

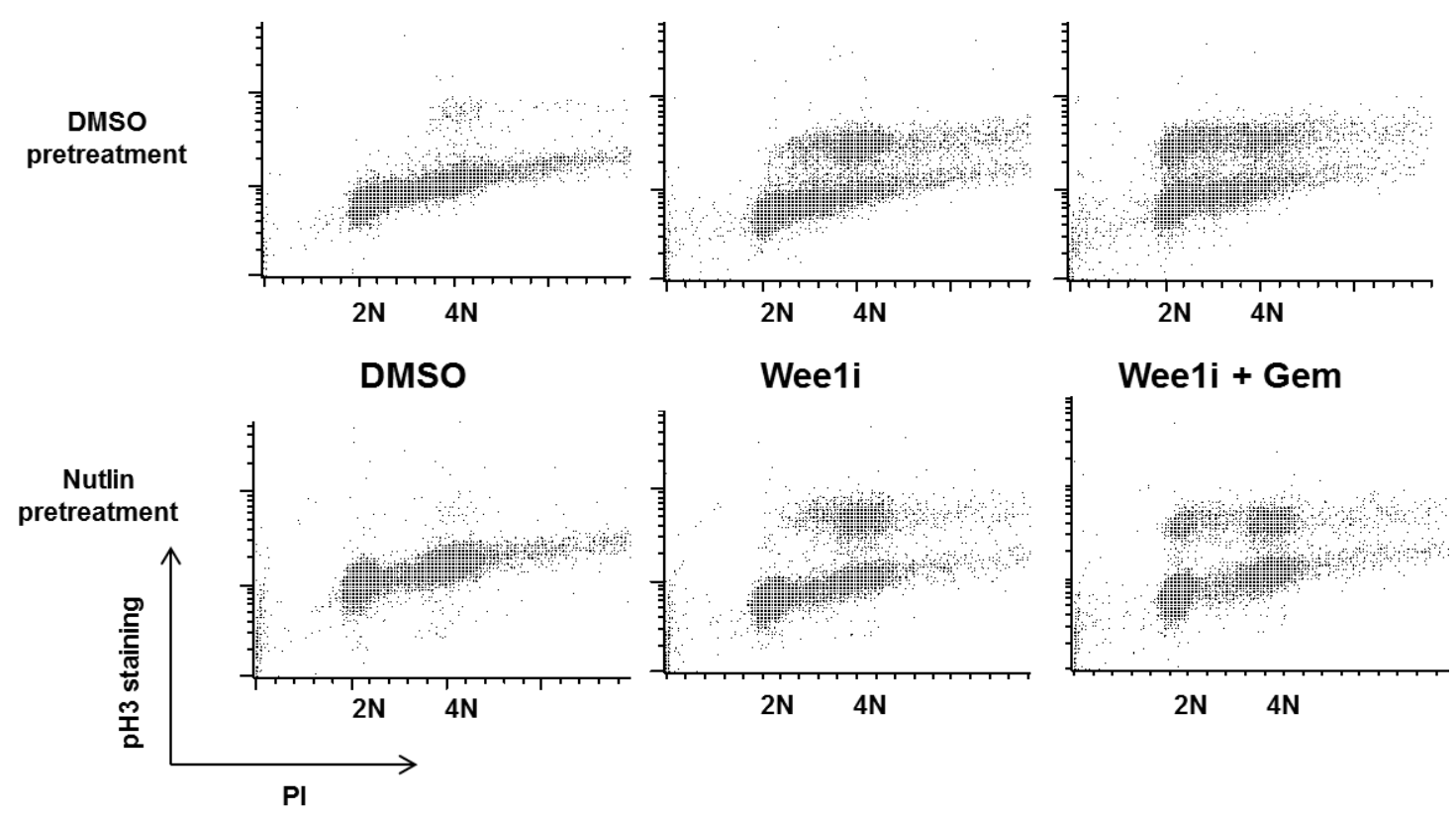

(b)
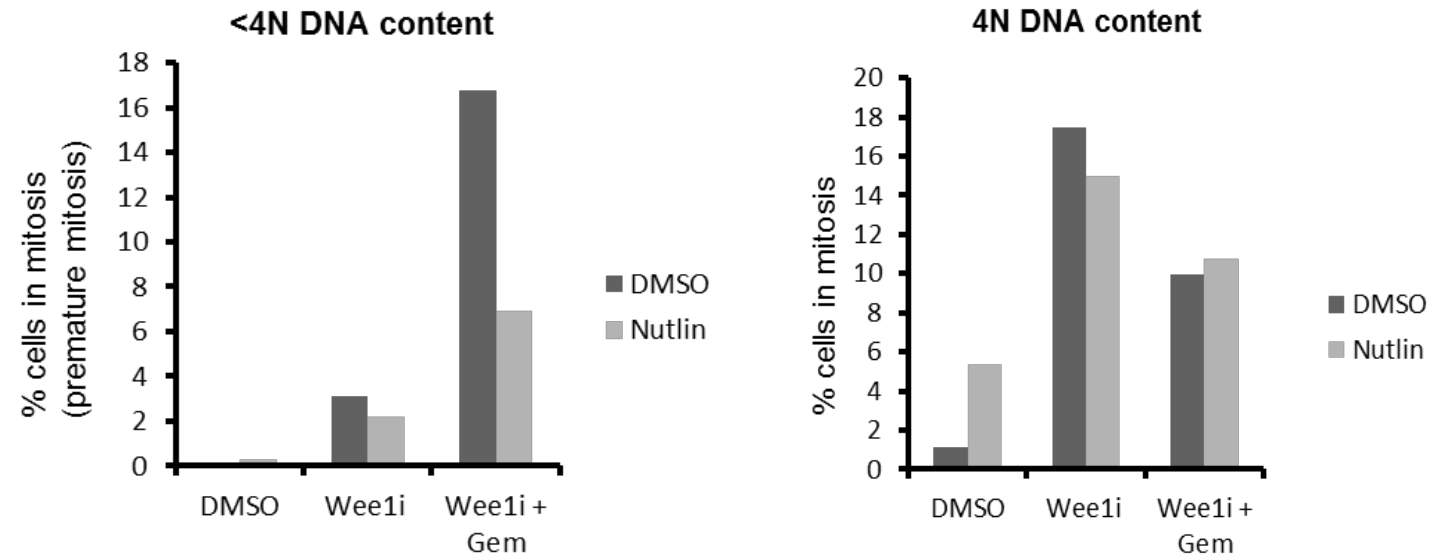

Figure V-XVIII Nutlin-3 pretreatment protects cells from premature mitosis caused by Wee1 inhibitor and gemcitabine treatment.

U2OS cells were treated with $8 \mu \mathrm{M}$ nutlin-3 for $24 \mathrm{~h}$, followed by treatment with $1 \mu \mathrm{M}$ Wee1 inhibitor, $300 \mathrm{nM}$ gemcitabine and $8 \mu \mathrm{M}$ nutlin-3. Cells were fixed, stained with phospho- $\mathrm{H} 3$ and Propidium lodide (PI) and analyzed by flow cytometry. 


\section{Discussion}

\section{VI.1 Emergence of combination therapy}

Gemcitabine has been the cornerstone of pancreatic tumor treatment. However, despite being the standard treatment regimen, the response rate of patients and survival has not been encouraging. This has been due to two major factors- first, tumor detection is possible only at advanced stages, and second, tumors develop resistance to gemcitabine (Burris 1997, M. P. Kim and Gallick 2008). Being a nucleoside analog, gemcitabine elicits excessive DNA damage response in tumors, eventually killing them (For mechanism, refer to section II.4.I).

Inhibition of regulators of intra-S and G2 checkpoints is currently being used extensively to sensitize tumor cells against chemotherapeutics e.g, combination of ATR inhibition with cisplatin increases the sensitivity of tumors towards cisplatin (Yazlovitskaya and Persons 2003, Perez et al. 2006, Ma et al. 2013). Abrogation of the ATR, Chk1 and Wee1 checkpoint kinases is known to sensitize tumor cells against gemcitabine (Prevo et al. 2012, Zabludoff et al. 2008, Rajeshkumar et al. 2011). In this project, we performed a comparative study in pancreatic tumor cells to identify checkpoint kinase candidate which is the most effective in sensitizing these tumor cells towards gemcitabine. For the inhibition of respective kinases, we used following inhibitors- VE-821 (ATR inhibitor), SB218078 (Chk1 inhibitor) and MK-1775 (Wee1 inhibitor). We evaluated their effects by several molecular biological methods. We identify a combination cocktail of gemcitabine with the Wee1 inhibitor MK-1775 to be the most potent in sensitizing pancreatic tumor cells against gemcitabine.

Our findings complement previous studies which show that gemcitabine treatment along with concomitant checkpoint kinase inhibition increases the DNA damage response of the cells. We, however, do not observe any changes in gemcitabine sensitivity upon ATR knockdown in Panc1 cells. This could be due to a different isoform of ATR that is expressed in the pancreas, and might substitute for ATR loss (Mannino et al. 2001). Also, we found Chk1 knockdown in U2OS and BxPC3 cells does not augment DNA damage as compared to gemcitabine alone. The reason for such observation could be saturation of $\mathrm{YH} 2 \mathrm{AX}$ signal due to gemcitabine. As a result, Chk1 inhibition does not potentiate $\mathrm{yH} 2 \mathrm{AX}$ intensity.

In addition, growth of the cells with combination treatment is hampered in long- term survival assay, with the exception of Chk1 inhibition in combination with gemcitabine in 
Panc1 cells. It is known that Chk1 inhibitors, specifically UCN-01 and AZD7762, can cause activation of the Erk1/2 and ATM pathway (Dent et al. 2011) which can contribute to increased survivability of cells. It has been reported that activation of Erk can have different effects on growth, cell cycle arrest, apoptosis and induction of drug resistance in different cell lineages which can depend on the presence of p53 and the expression of lineage specific factors (McCubrey et al. 2007). The survival effect of Chk1 inhibitor that we observed in the assay could be a consequence of Erk1/2 activity. Another Chk1 inhibitor SCH 900776 has been reported to phenocopy the effects of Chk1 knockdown (Guzi et al. 2011). Moreover, we observed that knockdown of Chk1 alone induces activation of $\mathrm{yH} 2 \mathrm{AX}$ while its inhibition does not. We propose the reason for such observation could be the kinase-independent function of Chk1. It is possible that inhibition of Chk1 still allows it to maintain fork progression through translesion DNA synthesis, a supplementary DNA replication process, while its knockdown cannot (Speroni et al. 2012).

Altogether, we find that out of three checkpoint kinase inhibitors, MK-1775 (Wee1 inhibitor) shows higher potency in sensitizing the pancreatic tumor cells towards gemcitabine.

\section{VI.2 Crosstalk between Wee1 and ATR-Chk1 pathway}

Gemcitabine generates DNA damage response by activating ATR and/or ATM pathway. Also, Wee1 inhibition elicits DNA damage response (Domínguez-Kelly et al. 2011). We analyzed the overall DNA damage response generated upon Wee1 inhibition and observed a crosstalk between Wee1 and ATR pathway. When Wee1 inhibition is combined with gemcitabine, ATR-Chk1 pathway is attenuated. However, $\mathrm{YH} 2 \mathrm{AX}$ intensity increases upon combination of the two drugs. Increased $\mathrm{YH} 2 \mathrm{AX}$ might be from the activity of other PIKKs such as ATM or DNA- PK which get activated upon DSB formation due to replication fork collapse (McNeely et al. 2010). Since silencing of ATR-Chk1 pathway is observed independent of the status of p53 upon inhibition of Wee1 along with gemcitabine (Figure V-IV), we postulate that attenuation of ATR-Chk1 activity is not mediated through p53. Moreover, the influence of Wee1 inhibition on ATR pathway could be one of the reasons for its high effectiveness in combination with gemcitabine.

Combination of Wee1 inhibitor with gemcitabine shows more PARP cleavage suggesting more apoptosis than gemcitabine treatment alone. Since caspase inhibition does not rescue Chk1 inactivation (Figure V-VII), so apoptosis could not reduce ATR activity. Therefore, reduction of ATR activity could, however, be a consequence of its inhibition. 
Previous reports show that inhibition of Wee1 in S-phase arrested cells leads to premature mitotic entry of cells resulting in mitotic catastrophe (Aarts et al. 2012). Interestingly, combination treatment in MCF7 cells, which resist premature mitosis when Wee1 is inhibited in the presence of gemcitabine, does not decrease ATR activity (Figure V-VIII).

It is not known yet how Wee1 can regulate the ATR pathway in humans. Conversely, however, in Xenopus, Chk1 has been implicated in activation of Wee1 (J. Lee, Kumagai, and Dunphy 2001). Our observation of reduction in phosphorylation of Chk1 only after 12 h (Figure V-VI) suggests that Wee1 might not be directly involved in regulation of ATR pathway. This led us to assess the role of Wee1 substrates, Cdks, in inactivating this DNA damage pathway.

\section{VI.3 Decreased activation of ATR-Chk1 pathway is mediated through Cyclin-dependent kinases and Polo- like kinase 1}

We show that Cdks negatively control the ATR-Chk1 pathway (Figure V-IX). Wee1 phosphorylates Cdks at $\mathrm{Y} 15$ and inhibits their activity. To investigate the role of Cdks in modulating the ATR pathway, we inhibited Cdks with Roscovitine, which selectively inhibits Cdk1, Cdk2 and Cdk5 by competing for their ATP-binding domain (Bach et al. 2005). Our results show that the regulation of ATR-Chk1 pathway is mediated through the activity of Cdks (Figure V-IX). Furthermore, we show that Cdk1 inhibition could rescue the inhibition of ATR-Chk1 pathway upon Wee1 inhibition with gemcitabine treatment (Figure $\mathbf{V}-\mathbf{X})$. Therefore, we can say that Cdk1 plays an important role in mediating inactivation of ATR pathway. This is a novel finding that has several implications in tumor therapy. Different Cdks have been implicated in several tumors for their diagnostic/ prognostic value or aberrant expression (Cicenas and Valius 2011). We postulate that use of Wee1 inhibitor along with gemcitabine might be much more toxic in tumor cells expressing high levels of Cdks, therefore, it can serve as an efficient way of eliminating these cells.

There is no evidence of direct interaction of Cdks with ATR; therefore, we tested whether this response is mediated through Cdk substrates. Mus81-Eme1 endonuclease complex has been implicated in processing of DSB and proposed to be activated by Cdk2 (Domínguez-Kelly et al. 2011). Based on these findings, we speculated that Mus81 might be responsible for ATR-Chk1 inhibition once Cdk1 becomes hyperactivated due to Wee1 depletion. However, we observed that Mus81 removal in the presence of gemcitabine decreased the phosphorylation of Chk1 as well as H2AX (Figure V-XI), thereby demonstrating the role of Mus81 in sustaining the activation of DNA damage response. In 
their study, Domínguez-Kelly and colleagues, had observed a reduction in the DNA damage response, generated by Wee1 removal, upon co-depletion of Mus81 (Domínguez-Kelly et al. 2011). This possibly supports our observation of decreased $\mathrm{YH} 2 \mathrm{AX}$ and phospho-Chk1 upon Mus81 removal in the presence of gemcitabine. It can be explained based on the fact that Wee1 inhibition leads to Cdk hyperactivation, which leads to deregulation of DNA replication resulting in the formation of abnormal DNA structures (Beck et al. 2012); these structures are resolved by Mus81-Eme1 (DomínguezKelly et al. 2011). It is possible that during the processing of these structures, ssDNA is generated, which fortifies DNA damage response signaling (Sugawara and Haber 1992).

Aditionally, $\mathrm{pRb}$ has been reported to be regulated by Cdks. Cdks phosphorylate pRb, thereby inactivate its binding to E2F and increase E2F-mediated transcription (Lundberg and Weinberg 1998). Therefore, we suspected that $\mathrm{pRb}$ might be involved in controlling the ATR-Chk1 pathway either through its substrate E2F or directly. E2F, being a transcription factor can possibly mediate its effect on this pathway through repression of ATR transcription (Ren et al. 2002, Stevens and La Thangue 2004). However, our data shows that of ATR-Chk1 pathway is E2F-independent as mRNA expression of ATR does not change upon Wee1 inhibition with gemcitabine (Figure V-XII). Moreover, pRb does not modulate this pathway, as even in the absence of functional pRb, inhibition of Cdk rescues the phosphorylation of Chk1. From here, we can say that the toxic effects of the combination of Wee1 inhibition with gemcitabine are independent of the status of $\mathrm{pRb}$. Therefore, tumors having mutated or inactive pRb (Wiest et al. 2002, Xiao et al. 2002) can respond efficiently to this treatment thereby expanding the prospects of this combination therapy.

There have been reports which link the activity of Plk1 with Cdks. In humans, Cdk1 has been reported to prime the Plk1 substrates by phosphorylating them. The 'primed' substrates are then recognized by Plk1, which facilitates the activation of Plk1 (K. S. Lee et al. 2008). In S. cerevisiae, Cdk1 maintains the stability of Plk1 by phosphorylation at Thr23 (Simpson-Lavy and Brandeis 2011). Furthermore, Plk1 has been implicated in phosphorylation of Claspin and its subsequent degradation, thereby, preventing activation of Chk1 in response to DNA damage signal (Mailand et al. 2006, Mamely et al. 2006, Peschiaroli et al. 2006). We observe that inhibition of Plk1 can rescue the attenuated ATR-Chk1 activity caused by Wee1 inhibition in the presence of gemcitabine (Figure V-XIII). This indicates the involvement of Plk1 in inactivating ATR-Chk1 pathway. We show that phosphorylation of Plk1 at Thr210 which is mediated by Aurora-A kinase and is a marker for the activation of Plk1 (Seki et al. 2008), increases upon Wee1 inhibition in gemcitabine-treated cells (Figure V-XIII). This strengthens the idea of an immediate role 
of Plk1 in mediating inactivation of ATR-Chk1 pathway. Moreover, activation of Plk1 upon inhibition of Cdks is reduced. This finding supports the previous literature that Cdks assist in Plk1 activation. We also find decreased Thr210 phosphorylation on Plk1 upon Plk1 inhibition (Figure V-XIII). The Plk1 inhibitor, GSK461364 is an ATP-competitive inhibitor of Plk1 (Olmos et al. 2011), however, how this inhibitor affects Aurora-A kinase mediated activation of Plk1 remains to be understood. We also show that indeed Plk1 mediates inactivation of Chk1 through Claspin degradation (Figure V-XIV).

Inhibition of Wee1 with gemcitabine increases the Plk1 activation (Figure V-XIII), and the activated Plk1 drives the cells into mitosis (Seki et al. 2008) which can be a reason for mitotic catastrophe. It is because the cells undergo replicative stress with gemcitabine but upon inhibition of Wee1, Plk1 activation causes the cells with unreplicated DNA to enter into mitosis resulting in cell death. Now, we show Plk1 also mediates inactivation of ATRChk1 pathway. Thus, altogether, unregulated Plk1 activity in already stressed cells is lethal to the cells.

Plk1 activation requires Bora protein (Seki et al. 2008) and the protein levels of Bora are found to be high in some cases of pancreatic cancer (http://www.proteinatlas.org/ENSG00000136122/cancer dated140815). From here, we predict that the combination of Wee1 inhibitor and gemcitabine in these cells can prove to be quite toxic due to high activation of Plk1 and thus, resulting inactivation of ATR-Chk1 pathway. Furthermore, it may be worth testing if removal of Bora from the cells produces the same effects as inhibition of Plk1.

From the results obtained, we propose the following model-

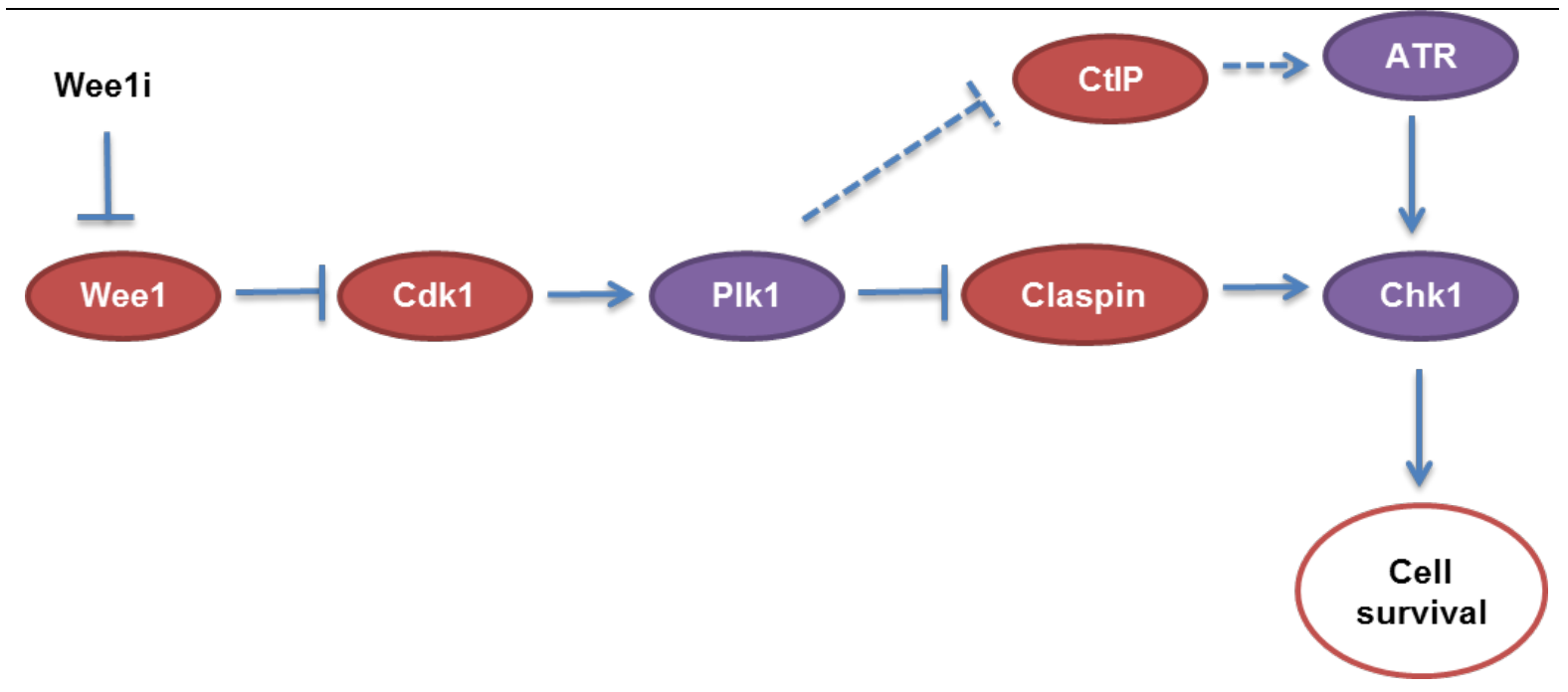

Figure VI-I Wee1 inhibition hampers ATR-Chk1 activity.

Inhibition of Wee1 hyperactivates Cdk1 which, in turn, promotes Plk1 activation. Activated Plk1 leads to Claspin degradation; consequently ATR-Chk1 activity is attenuated. PIk1 might regulate degradation of CtIP, which then leads to attenuated ATR activity. 


\section{VI.4 Attenuating the side-effects of combination treatment by nutlin-3 pretreatment}

The nucleoside analogues and other drugs that induce replicative stress lead to undesired effects by causing the destruction of rapidly dividing normal cells. These include hematopoetic cells (mostly in the bone marrow) as well as the epithelia of the gut, and hair follicles (Galmarini, Mackey, and Dumontet 2002). Indeed, the dose-limiting toxicity of gemcitabine causes myelosuppression, as for many other DNA-damaging chemotherapeutics (Fossella et al. 1997). This raises the need to provide specific protection to normal cells.

To protect normal dividing cells from the toxic effects of chemotherapy, the checkpoint machinery can be exploited. In normal cells, p53 is present in wild-type form while in most of the cancer cells, it is mutated or absent (Nigro et al. 1989). Wild-type p53 is activated by nutlin-3; however, the drug has no effect on mutant p53 (Coll-Mulet et al. 2006). This differential activity of nutlin-3 has been utilized to protect the healthy cells from cytotoxicity caused by chemotherapeutic drugs. In the work done by Kranz and colleagues, nutlin-3 protects the wild-type p53 containing cells against gemcitabine. Gemcitabine induces DNA damage and apoptosis in cancer cells irrespective of their p53 status, nevertheless when these cells are pretreated with non-toxic amounts of nutlin-3, cells with wild-type p53 show reduced DNA damage response and cell death (Kranz and Dobbelstein 2006). Activation of wild-type p53 causes transient cell cycle arrest in G1 or G2, mostly through the induction of the CDKN1A gene, encoding the cyclin dependent kinase inhibitor p21, with subsequent hypophosphorylation of the retinoblastoma family of proteins and repression of E2F target gene (Polager and Ginsberg 2009). If this happens, the cells will be protected against therapeutic regimens that rely on the enhancement of replicative stress, e. g. nucleoside analogues. We find that nutlin-3 pretreatment could rescue the wild-type p53 containing cells from lethal effects of the combination of Wee1 inhibition and gemcitabine. Apart from attenuating the DNA damage response and apoptosis, we observe that nutlin-3 pretreatment could also reduce the protein levels of phosho- $\mathrm{H} 3, \mathrm{a}$ mitotic marker (Figure V-XV). This indicates that nutlin-3 could protect the cells from entering into mitosis, which is induced by Wee1 inhibition. As Wee1 inhibition leads to premature entry of cells into mitosis, we measured the number of cells entering into mitosis prematurely using mitotic marker phospho-H3 and cell cycle analysis. Our results show that premature mitosis decrease significantly in cells pretreated with nutlin-3 (Figure V-XVIII) which can be due to G1 or G2 arrest caused by induction of p21. As expected, the protein levels of Mdm2 increased upon treatment with nutlin-3, because nutlin-3 
protects Mdm2 from degradation as well as causes increase in Mdm2 transcription (Leeuwen et al. 2011). However, for unknown reasons, combining Wee1 inhibitor (either alone or with gemcitabine) with nutlin-3 did not stabilize Mdm2. We also show that wildtype p53 is required for the protective function of nutlin-3 as the cells without p53 remain unaffected by the addition of nutlin-3 (Figure V-XVI).

This study reflects the beneficial use of Mdm2 antagonists in clinics. When treating the patients with combination therapy, addition of these antagonists at sub-lethal dose could help reduce side-effects of the combination treatment.

\section{VI.5 Conclusions}

Our data shows that Wee1 inhibition decreases activity of Chk1; however, there are studies which find cooperative effects when using inhibitors of Chk1 and Wee1 simultaneously for cancer treatment (Davies et al. 2011, Guertin et al. 2012). Here, we propose that this cooperativity between Chk1 and Wee1 inhibitor might explained by the timing of the Chk1 inactivation. As, we observe decrease in Chk1 activity upon Wee1 inhibition only after a couple of hours. For the increased sensitization of cells, inhibition of Chk1 might be required immediately after the addition of nucleoside analogues. Moreover, the cell type and the choice of concomitant chemotherapies may also affect the cooperativity between inhibitors of Wee1 and Chk1. In any case, we show here that at least in the presence of gemcitabine, Chk1 and ATR can be attenuated by Wee1 inhibitor alone.

Our results strongly suggest that Wee1 inhibition kills cancer cells not only by premature entry of cells to mitosis and resulting mitotic catastrophe (Aarts et al. 2012) but also by augmenting replication stress through impairment of ATR-Chk1 signaling. This unique combination of cytotoxic mechanisms, triggered through a single target, provides an explanation for the remarkable cytotoxic efficacy of Wee1 inhibitors. Wee1 inhibitors represent promising anti-cancer drug candidates (Rajeshkumar et al. 2011, Do et al. 2013) and are currently being tested in clinical trials of phases I and II ( $\mathrm{NCl}$ Clinical Trials).

Chemotherapies that enhance replicative stress above the endogenous level also affect non-malignant cells, presumably resulting in unwanted toxicities. Therefore, to protect normal cells from replicative stress without compromising the cytotoxic effects on tumor cells, it is necessary to exploit characteristic differences between malignant and normal cells. Our results suggest that Nutlin-3 pretreatment provides protection to p53-proficient 
cells against the combination of Wee1 inhibitor and gemcitabine. We propose that this approach can possibly be transferred to the clinics to evaluate the protective effects of Mdm2 antagonists against replicative stress.

\section{VI.6 Future perspectives}

Recent studies have shown the regulation of Wee1 by miRNAs. In some tumors, levels of miRNAs suppressing Wee1 expression have found to be up-regulated (Butz et al. 2010, Bhattacharya et al. 2013), treatment of such tumors with nucleoside analogs might be beneficial.

To further study the mechanism leading to down-regulation of ATR activity, the protein called CtIP seems to be a good candidate. Donnianni and colleagues have shown in budding yeast that Sae2, which is a functional ortholog of human CtIP, is regulated by Plk1 (cdc5 in yeast) and is involved in silencing of DNA damage signaling (Donnianni et al. 2010). In S. cerevisiae, Sae2 negatively regulates checkpoint signaling by modifying the association of MRX at damaged DNA sites. Depletion of Sae2 in the cells prevents the Mec1 (ATR)- and Tel1 (ATM)-dependent signaling to turn off and interrupts with the disassembly of Mre11 foci at DNA breaks (Clerici et al. 2006). In contrast, in one of the studies in humans, CtIP has been reported to resect DSBs and thus, recruit RPA and ATR to the DSB sites, subsequently leading to ATR activation (Sartori et al. 2007). In a recent study, CtIP is shown to be required for sustaining the ATR-Chk1 pathway while it is not necessarily required for initiating its activation (Kousholt et al. 2012). However, both of the studies in humans present CtIP as a positive regulator of the checkpoint signaling pathway. Study can be performed to determine the role of CtIP in DNA damage signaling upon combination treatment and its regulation by Plk1. It is possible that phosphorylation of CtIP by Plk1 is responsible for different responses of CtIP upon DNA damage. It might be possible that both the effects are mediated by CtIP in a time-dependent fashion. Initially, CtIP is required for maintaining ATR-Chk1 activation but once the resection is completed, it mediates to turn off this pathway. Moreover, CtIP has been found to be phosphorylated by Cdks and this phosphorylation along with another phosphorylation mediates binding of Pin1 protein to CtIP. Pin1 isomerization with CtIP facilitates its degradation (Sartori and Steger 2013). Also, Plk1 has been found to stabilize Pin1 protein (Eckerdt et al. 2005). Therefore, we speculated that upon inhibition of Wee1, hyperactive Cdks phosphorylate CtIP and activated Plk1 stabilize Pin1, which ultimately facilitates proteasomal degradation of CtIP. 
A previous study has shown that replicative stress caused by Wee1 inhibition due to deregulated Cdk activity can be suppressed by supplementing the cells with nucleosides. Inhibition of Wee1 causes increased Cdk activity which leads to augmented initiation of replication, resulting in scarcity of nucleotide and reduced replication fork speed, followed by Mus81-mediated DNA double strand breakage. When nucleosides are added, they stabilize the fork speed and reduce DNA DSB formation (Beck et al. 2012). Based on these observations, it can be tested if the addition of nucleosides has any effect on the ATR-Chk1 activity upon addition of Wee1 inhibitor in gemcitabine-treated cells.

Since a long time, combinatorial approaches have been applied in classical chemotherapeutical regimens, including nucleoside analogues, platinum compounds, and/or topoisomerase inhibitors. Combinations of these drugs are usually more effective than single drugs and have been tested in multiple clinical trials. However, the knowledge on how these drugs affect each other's efficacy at molecular level is very limited. Therefore, we consider that investigating in this area may promote the development of more efficient combinatorial regimens. 


\section{References}

Aarts, Marieke, Rachel Sharpe, Isaac Garcia-Murillas, Heidrun Gevensleben, Melissa S. Hurd, Stuart D. Shumway, Carlo Toniatti, Alan Ashworth, and Nicholas C. Turner. 2012a. "Forced Mitotic Entry of S-Phase Cells as a Therapeutic Strategy Induced by Inhibition of WEE1." Cancer Discovery 2 (6): 524-39. doi:10.1158/21598290.CD-11-0320.

Albain, Kathy S., Shona M. Nag, German Calderillo-Ruiz, Johann P. Jordaan, Antonio C. Llombart, Anna Pluzanska, Janusz Rolski, et al. 2008. "Gemcitabine Plus Paclitaxel Versus Paclitaxel Monotherapy in Patients With Metastatic Breast Cancer and Prior Anthracycline Treatment." Journal of Clinical Oncology 26 (24): 3950-57. doi:10.1200/JCO.2007.11.9362.

Archambault, Vincent, and David M Glover. 2009. "Polo-like Kinases: Conservation and Divergence in Their Functions and Regulation." Nature Reviews. Molecular Cell Biology 10 (4): 265-75. doi:10.1038/nrm2653.

Asano, Takayuki, Yixin Yao, Jijiang Zhu, Donghui Li, James L Abbruzzese, and Shrikanth A G Reddy. 2004. "The PI 3-kinase/Akt Signaling Pathway Is Activated due to Aberrant Pten Expression and Targets Transcription Factors NF-kappaB and CMyc in Pancreatic Cancer Cells." Oncogene 23 (53): 8571-80. doi:10.1038/sj.onc.1207902.

Ashwell, Susan, and Sonya Zabludoff. 2008. "DNA Damage Detection and Repair Pathways-Recent Advances with Inhibitors of Checkpoint Kinases in Cancer Therapy." Clinical Cancer Research 14 (13): 4032-37. doi:10.1158/10780432.CCR-07-5138.

Azorsa, David O., Irma M. Gonzales, Gargi D. Basu, Ashish Choudhary, Shilpi Arora, Kristen M. Bisanz, Jeffrey A. Kiefer, et al. 2009. "Synthetic Lethal RNAi Screening Identifies Sensitizing Targets for Gemcitabine Therapy in Pancreatic Cancer." Journal of Translational Medicine 7 (1): 43. doi:10.1186/1479-5876-7-43.

Bach, Stéphane, Marie Knockaert, Jens Reinhardt, Olivier Lozach, Sophie Schmitt, Blandine Baratte, Marcel Koken, et al. 2005. "Roscovitine Targets, Protein Kinases and Pyridoxal Kinase." Journal of Biological Chemistry 280 (35): 31208-19. doi:10.1074/jbc.M500806200.

Baker, C H, J Banzon, J M Bollinger, J Stubbe, V Samano, M J Robins, B Lippert, E Jarvi, and R Resvick. 1991. '2'-Deoxy-2'-Methylenecytidine and 2'-Deoxy-2',2'Difluorocytidine 5'-Diphosphates: Potent Mechanism-Based Inhibitors of Ribonucleotide Reductase." Journal of Medicinal Chemistry 34 (6): 1879-84.

Bakkenist, Christopher J, and Michael B Kastan. 2003. "DNA Damage Activates ATM through Intermolecular Autophosphorylation and Dimer Dissociation." Nature 421 (6922): 499-506. doi:10.1038/nature01368.

Bao, Shideng, Randal S. Tibbetts, Kathryn M. Brumbaugh, Yanan Fang, D. Ashley Richardson, Ambereen Ali, Susan M. Chen, Robert T. Abraham, and Xiao-Fan Wang. 2001. "ATR/ATM-Mediated Phosphorylation of Human Rad17 Is Required for Genotoxic Stress Responses." Nature 411 (6840): 969-74. doi:10.1038/35082110.

Barrett, Rachel M A, Toby P Osborne, and Sally P Wheatley. 2009. "Phosphorylation of Survivin at Threonine 34 Inhibits Its Mitotic Function and Enhances Its Cytoprotective Activity." Cell Cycle (Georgetown, Tex.) 8 (2): 278-83.

Bartek, Jiri, and Jiri Lukas. 2001. "Mammalian G1- and S-Phase Checkpoints in Response to DNA Damage." Current Opinion in Cell Biology 13 (6): 738-47. doi:10.1016/S0955-0674(00)00280-5.

Baxter, G. D., and M. F. Lavin. 1992. "Specific Protein Dephosphorylation in Apoptosis Induced by lonizing Radiation and Heat Shock in Human Lymphoid Tumor Lines." The Journal of Immunology 148 (6): 1949-54. 
Beck, Halfdan, Viola Nähse-Kumpf, Marie Sofie Yoo Larsen, Karen A. O'Hanlon, Sebastian Patzke, Christian Holmberg, Jakob Mejlvang, et al. 2012a. "CyclinDependent Kinase Suppression by WEE1 Kinase Protects the Genome through Control of Replication Initiation and Nucleotide Consumption." Molecular and Cellular Biology 32 (20): 4226-36. doi:10.1128/MCB.00412-12.

Bellosillo, Beatriz, Neus Villamor, Armando López-Guillermo, Silvia Marcé, Francesc Bosch, Elias Campo, Emili Montserrat, and Dolors Colomer. 2002. "Spontaneous and Drug-Induced Apoptosis Is Mediated by Conformational Changes of Bax and Bak in B-Cell Chronic Lymphocytic Leukemia." Blood 100 (5): 1810-16. doi:10.1182/blood-2001-12-0327.

Bhattacharya, A., U. Schmitz, O. Wolkenhauer, M. Schönherr, Y. Raatz, and M. Kunz. 2013. "Regulation of Cell Cycle Checkpoint Kinase WEE1 by miR-195 in Malignant Melanoma." Oncogene 32 (26): 3175-83. doi:10.1038/onc.2012.324.

Blasina, A, I V de Weyer, M C Laus, W H Luyten, A E Parker, and C H McGowan. 1999. "A Human Homologue of the Checkpoint Kinase Cds1 Directly Inhibits Cdc25 Phosphatase." Current Biology: CB 9 (1): 1-10.

Bolderson, Emma, Jennifer Scorah, Thomas Helleday, Carl Smythe, and Mark Meuth. 2004. "ATM Is Required for the Cellular Response to Thymidine Induced Replication Fork Stress." Human Molecular Genetics 13 (23): 2937-45. doi:10.1093/hmg/ddh316.

Bondar, Victor M., Bridget Sweeney-Gotsch, Michael Andreeff, Gordon B. Mills, and David J. McConkey. 2002. "Inhibition of the Phosphatidylinositol 3'-Kinase-AKT Pathway Induces Apoptosis in Pancreatic Carcinoma Cells in Vitro and in Vivo." Molecular Cancer Therapeutics 1 (12): 989-97.

Bulavin, D V, Y Higashimoto, I J Popoff, W A Gaarde, V Basrur, O Potapova, E Appella, and A J Fornace Jr. 2001. "Initiation of a G2/M Checkpoint after Ultraviolet Radiation Requires p38 Kinase." Nature 411 (6833): 102-7. doi:10.1038/35075107.

Burris, H A, 3rd, M J Moore, J Andersen, M R Green, M L Rothenberg, M R Modiano, M C Cripps, R K Portenoy, A M Storniolo, P Tarassoff, R Nelson, F A Dorr, C D Stephens, and D D Von Hoff. 1997a. "Improvements in Survival and Clinical Benefit with Gemcitabine as First-Line Therapy for Patients with Advanced Pancreas Cancer: A Randomized Trial." Journal of Clinical Oncology: Official Journal of the American Society of Clinical Oncology 15 (6): 2403-13.

Burris, H. A., M. J. Moore, J. Andersen, M. R. Green, M. L. Rothenberg, M. R. Modiano, M. C. Cripps, R. K. Portenoy, A. M. Storniolo, P. Tarassoff, R. Nelson, F. A. Dorr, C. D. Stephens, and D. D. Von Hoff. 1997b. "Improvements in Survival and Clinical Benefit with Gemcitabine as First-Line Therapy for Patients with Advanced Pancreas Cancer: A Randomized Trial." Journal of Clinical Oncology: Official Journal of the American Society of Clinical Oncology 15 (6): 2403-13.

Burris, H. A., M. J. Moore, J. Andersen, M. R. Green, M. L. Rothenberg, M. R. Modiano, M. C. Cripps, R. K. Portenoy, A. M. Storniolo, P. Tarassoff, R. Nelson, F. A. Dorr, C. D. Stephens, and D. D. Von Hoff. 1997. "Improvements in Survival and Clinical Benefit with Gemcitabine as First-Line Therapy for Patients with Advanced Pancreas Cancer: A Randomized Trial." Journal of Clinical Oncology 15 (6): 240313. http://jco.ascopubs.org/content/15/6/2403.

Butz, Henriett, István Likó, Sándor Czirják, Péter Igaz, Mohammed Munayem Khan, Vladimir Zivkovic, Katalin Bálint, Márta Korbonits, Károly Rácz, and Attila Patócs. 2010. "Down-Regulation of Wee1 Kinase by a Specific Subset of microRNA in Human Sporadic Pituitary Adenomas." The Journal of Clinical Endocrinology and Metabolism 95 (10): E181-191. doi:10.1210/jc.2010-0581.

Carvajal, Daisy, Christian Tovar, Hong Yang, Binh T. Vu, David C. Heimbrook, and Lyubomir T. Vassilev. 2005. "Activation of p53 by MDM2 Antagonists Can Protect Proliferating Cells from Mitotic Inhibitors." Cancer Research 65 (5): 1918-24. doi:10.1158/0008-5472.CAN-04-3576. 
Castedo, Maria, Arnaud Coquelle, Sonia Vivet, Ilio Vitale, Audrey Kauffmann, Philippe Dessen, Marie O Pequignot, et al. 2006. "Apoptosis Regulation in Tetraploid Cancer Cells." The EMBO Journal 25 (11): 2584-95. doi:10.1038/sj.emboj.7601127.

Chehab, N H, A Malikzay, M Appel, and T D Halazonetis. 2000. "Chk2/hCds1 Functions as a DNA Damage Checkpoint in $\mathrm{G}(1)$ by Stabilizing p53." Genes \& Development 14 (3): 278-88.

Chehab, Nabil H., Asra Malikzay, Michael Appel, and Thanos D. Halazonetis. 2000. "Chk2/hCds1 Functions as a DNA Damage Checkpoint in G1 by Stabilizing p53." Genes \& Development 14 (3): 278-88.

Cheng, Kin-Yip, Edward D Lowe, John Sinclair, Erich A Nigg, and Louise N Johnson. 2003. "The Crystal Structure of the Human Polo-like Kinase-1 Polo Box Domain and Its Phospho-Peptide Complex." The EMBO Journal 22 (21): 5757-68. doi:10.1093/emboj/cdg558.

Chini, Claudia Christiano Silva, and Junjie Chen. 2003. "Human Claspin Is Required for Replication Checkpoint Control." The Journal of Biological Chemistry 278 (32): 30057-62. doi:10.1074/jbc.M301136200.

Chowdhury, Dipanjan, Michael-Christopher Keogh, Haruhiko Ishii, Craig L Peterson, Stephen Buratowski, and Judy Lieberman. 2005. "Gamma-H2AX Dephosphorylation by Protein Phosphatase 2A Facilitates DNA Double-Strand Break Repair." Molecular Cell 20 (5): 801-9. doi:10.1016/j.molcel.2005.10.003.

Chowdhury, Dipanjan, Xingzhi Xu, Xueyan Zhong, Fariyal Ahmed, Jianing Zhong, Ji Liao, Derek M Dykxhoorn, David M Weinstock, Gerd P Pfeifer, and Judy Lieberman. 2008. "A PP4-Phosphatase Complex Dephosphorylates Gamma-H2AX Generated during DNA Replication." Molecular Cell 31 (1): 33-46. doi:10.1016/j.molcel.2008.05.016.

Cicenas, Jonas, and Mindaugas Valius. 2011. "The CDK Inhibitors in Cancer Research and Therapy." Journal of Cancer Research and Clinical Oncology 137 (10): 140918. doi:10.1007/s00432-011-1039-4.

Clerici, Michela, Davide Mantiero, Giovanna Lucchini, and Maria Pia Longhese. 2006. "The Saccharomyces Cerevisiae Sae2 Protein Negatively Regulates DNA Damage Checkpoint Signalling." EMBO Reports $7 \quad$ (2): 212-18. doi:10.1038/sj.embor.7400593.

Cobb, Jennifer A, Lotte Bjergbaek, Kenji Shimada, Christian Frei, and Susan M Gasser. 2003. "DNA Polymerase Stabilization at Stalled Replication Forks Requires Mec1 and the RecQ Helicase Sgs1." The EMBO Journal 22 (16): 4325-36. doi:10.1093/emboj/cdg391.

Coll-Mulet, Llorenç, Daniel Iglesias-Serret, Antonio F. Santidrián, Ana M. Cosialls, Mercè de Frias, Esther Castaño, Clara Campàs, et al. 2006. "MDM2 Antagonists Activate p53 and Synergize with Genotoxic Drugs in B-Cell Chronic Lymphocytic Leukemia Cells." Blood 107 (10): 4109-14. doi:10.1182/blood-2005-08-3273.

Cortez, D, S Guntuku, J Qin, and S J Elledge. 2001. "ATR and ATRIP: Partners in Checkpoint Signaling." Science (New York, N.Y.) 294 (5547): 1713-16. doi:10.1126/science.1065521.

Costanzo, V, K Robertson, C Y Ying, E Kim, E Avvedimento, M Gottesman, D Grieco, and J Gautier. 2000. "Reconstitution of an ATM-Dependent Checkpoint That Inhibits Chromosomal DNA Replication Following DNA Damage." Molecular Cell 6 (3): 649-59.

Daskalakis, Michael, Tudung T. Nguyen, Carvell Nguyen, Per Guldberg, Gabriele Köhler, Pierre Wijermans, Peter A. Jones, and Michael Lübbert. 2002. "Demethylation of a Hypermethylated P15/INK4B Gene in Patients with Myelodysplastic Syndrome by 5-Aza-2'-Deoxycytidine (decitabine) Treatment." Blood 100 (8): 2957-64. doi:10.1182/blood.V100.8.2957.

Deer, Emily L., Jessica Gonzalez-Hernandez, Jill D. Coursen, Jill E. Shea, Josephat Ngatia, Courtney L. Scaife, Matthew A. Firpo, and Sean J. Mulvihill. 2010. 
"Phenotype and Genotype of Pancreatic Cancer Cell Lines." Pancreas 39 (4): 425-35. doi:10.1097/MPA.0b013e3181c15963.

Delacroix, Sinny, Jill M. Wagner, Masahiko Kobayashi, Ken-ichi Yamamoto, and Larry M. Karnitz. 2007. "The Rad9-Hus1-Rad1 (9-1-1) Clamp Activates Checkpoint Signaling via TopBP1." Genes \& Development 21 (12): 1472-77. doi:10.1101/gad.1547007.

Dent, Paul, Yong Tang, Adly Yacoub, Yun Dai, Paul B Fisher, and Steven Grant. 2011. "CHK1 Inhibitors in Combination Chemotherapy: Thinking beyond the Cell Cycle." Molecular Interventions 11 (2): 133-40. doi:10.1124/mi.11.2.11.

Dominguez-Kelly, R., Y. Martin, S. Koundrioukoff, M. E. Tanenbaum, V. A. J. Smits, R. H. Medema, M. Debatisse, and R. Freire. 2011. "Wee1 Controls Genomic Stability during Replication by Regulating the Mus81-Eme1 Endonuclease." The Journal of Cell Biology 194 (4): 567-79. doi:10.1083/jcb.201101047.

Domínguez-Kelly, Raquel, Yusé Martín, Stephane Koundrioukoff, Marvin E Tanenbaum, Veronique A J Smits, René H Medema, Michelle Debatisse, and Raimundo Freire. 2011a. "Wee1 Controls Genomic Stability during Replication by Regulating the Mus81-Eme1 Endonuclease." The Journal of Cell Biology 194 (4): 567-79. doi:10.1083/jcb.201101047.

Domínguez-Kelly, Raquel, Yusé Martín, Stephane Koundrioukoff, Marvin E. Tanenbaum, Veronique A. J. Smits, René H. Medema, Michelle Debatisse, and Raimundo Freire. 2011b. "Wee1 Controls Genomic Stability during Replication by Regulating the Mus81-Eme1 Endonuclease." The Journal of Cell Biology 194 (4): 567-79. doi:10.1083/jcb.201101047.

Donnianni, Roberto Antonio, Matteo Ferrari, Federico Lazzaro, Michela Clerici, Benjamin Tamilselvan Nachimuthu, Paolo Plevani, Marco Muzi-Falconi, and Achille Pellicioli. 2010. "Elevated Levels of the Polo Kinase Cdc5 Override the Mec1/ATR Checkpoint in Budding Yeast by Acting at Different Steps of the Signaling Pathway." PLoS Genet 6 (1): e1000763. doi:10.1371/journal.pgen.1000763.

Downs, Jessica A, Stéphane Allard, Olivier Jobin-Robitaille, Ali Javaheri, Andréanne Auger, Nathalie Bouchard, Stephen J Kron, Stephen P Jackson, and Jacques Côté. 2004. "Binding of Chromatin-Modifying Activities to Phosphorylated Histone H2A at DNA Damage Sites." Molecular Cell 16 (6): 979-90. doi:10.1016/j.molcel.2004.12.003.

Duxbury, M S, H Ito, M J Zinner, S W Ashley, and E E Whang. 2004. "Focal Adhesion Kinase Gene Silencing Promotes Anoikis and Suppresses Metastasis of Human Pancreatic Adenocarcinoma Cells." Surgery 135 (5): 555-62. doi:10.1016/j.surg.2003.10.017.

Duxbury, Mark S., Hiromichi Ito, Michael J. Zinner, Stanley W. Ashley, and Edward E. Whang. 2004. "Inhibition of Src Tyrosine Kinase Impairs Inherent and Acquired Gemcitabine Resistance in Human Pancreatic Adenocarcinoma Cells." Clinical Cancer Research 10 (7): 2307-18. doi:10.1158/1078-0432.CCR-1183-3.

Eckerdt, Frank, Juping Yuan, Krishna Saxena, Bernd Martin, Sven Kappel, Christine Lindenau, Andrea Kramer, et al. 2005. "Polo-like Kinase 1-Mediated Phosphorylation Stabilizes Pin1 by Inhibiting Its Ubiquitination in Human Cells." The Journal of Biological Chemistry 280 (44): 36575-83. doi:10.1074/jbc.M504548200.

el-Deiry, W S, T Tokino, V E Velculescu, D B Levy, R Parsons, J M Trent, D Lin, W E Mercer, K W Kinzler, and B Vogelstein. 1993. "WAF1, a Potential Mediator of p53 Tumor Suppression." Cell 75 (4): 817-25.

Elia, Andrew E H, Peter Rellos, Lesley F Haire, Jerry W Chao, Frank J Ivins, Katja Hoepker, Duaa Mohammad, Lewis C Cantley, Stephen J Smerdon, and Michael B Yaffe. 2003. "The Molecular Basis for Phosphodependent Substrate Targeting and Regulation of Plks by the Polo-Box Domain." Cell 115 (1): 83-95.

Elmore, Susan. 2007. "Apoptosis: A Review of Programmed Cell Death." Toxicologic Pathology 35 (4): 495-516. doi:10.1080/01926230701320337. 
Enders, Greg H. 2010. "Gauchos and Ochos: A Wee1-Cdk Tango Regulating Mitotic Entry." Cell Division 5 (1): 12. doi:10.1186/1747-1028-5-12.

Eom, Young-Woo, Mi Ae Kim, Seok Soon Park, Mi Jin Goo, Hyuk Jae Kwon, Seonghyang Sohn, Wook-Hwan Kim, Gyesoon Yoon, and Kyeong Sook Choi. 2005. "Two Distinct Modes of Cell Death Induced by Doxorubicin: Apoptosis and Cell Death through Mitotic Catastrophe Accompanied by Senescence-like Phenotype." Oncogene 24 (30): 4765-77. doi:10.1038/sj.onc.1208627.

Erkan, Mert, Jörg Kleeff, Irene Esposito, Thomas Giese, Knut Ketterer, Markus W Büchler, Nathalla A Giese, and Helmut Friess. 2005. "Loss of BNIP3 Expression Is a Late Event in Pancreatic Cancer Contributing to Chemoresistance and Worsened Prognosis." Oncogene 24 (27): 4421-32. doi:10.1038/sj.onc.1208642.

Espinosa, Enrique, Pilar Zamora, Jaime Feliu, and Manuel González Barón. 2003. "Classification of Anticancer Drugs-a New System Based on Therapeutic Targets." Cancer Treatment Reviews 29 (6): 515-23. doi:10.1016/S03057372(03)00116-6.

Ewald, B, D Sampath, and W Plunkett. 2008. "Nucleoside Analogs: Molecular Mechanisms Signaling Cell Death." Oncogene 27 (50): 6522-37. doi:10.1038/onc.2008.316.

Ewald, Brett, Deepa Sampath, and William Plunkett. 2007. "H2AX Phosphorylation Marks Gemcitabine-Induced Stalled Replication Forks and Their Collapse upon S-Phase Checkpoint Abrogation." Molecular Cancer Therapeutics 6 (4): 1239-48. doi:10.1158/1535-7163.MCT-06-0633.

Falck, J, N Mailand, R G Syljuåsen, J Bartek, and J Lukas. 2001. "The ATM-Chk2Cdc25A Checkpoint Pathway Guards against Radioresistant DNA Synthesis." Nature 410 (6830): 842-47. doi:10.1038/35071124.

Ferlay, Jacques, Hai-Rim Shin, Freddie Bray, David Forman, Colin Mathers, and Donald Maxwell Parkin. 2010. "Estimates of Worldwide Burden of Cancer in 2008: GLOBOCAN 2008." International Journal of Cancer. Journal International Du Cancer 127 (12): 2893-2917. doi:10.1002/ijc.25516.

Fernandez-Capetillo, Oscar, Alicia Lee, Michel Nussenzweig, and André Nussenzweig. 2004. "H2AX: The Histone Guardian of the Genome." DNA Repair 3 (8-9): 959-67. doi:10.1016/j.dnarep.2004.03.024.

Fossella, F. V., S. M. Lippman, D. M. Shin, P. Tarassoff, M. Calayag-Jung, R. PerezSoler, J. S. Lee, et al. 1997. "Maximum-Tolerated Dose Defined for Single-Agent Gemcitabine: A Phase I Dose-Escalation Study in Chemotherapy-Naive Patients with Advanced Non-Small-Cell Lung Cancer." Journal of Clinical Oncology 15 (1): 310-16.

Freedman, D A, L Wu, and A J Levine. 1999. "Functions of the MDM2 Oncoprotein." Cellular and Molecular Life Sciences: CMLS 55 (1): 96-107.

Furnari, Beth, Nicholas Rhind, and Paul Russell. 1997. "Cdc25 Mitotic Inducer Targeted by Chk1 DNA Damage Checkpoint Kinase." Science 277 (5331): 1495-97. doi:10.1126/science.277.5331.1495.

Galmarini, Carlos M, John R Mackey, and Charles Dumontet. 2002. "Nucleoside Analogues and Nucleobases in Cancer Treatment." The Lancet Oncology 3 (7): 415-24. doi:10.1016/S1470-2045(02)00788-X.

Garcia-Calvo, Margarita, Erin P. Peterson, Barbara Leiting, Rejean Ruel, Donald W. Nicholson, and Nancy A. Thornberry. 1998. "Inhibition of Human Caspases by Peptide-Based and Macromolecular Inhibitors." Journal of Biological Chemistry 273 (49): 32608-13. doi:10.1074/jbc.273.49.32608.

Genini, D, S Adachi, Q Chao, D W Rose, C J Carrera, H B Cottam, D A Carson, and L M Leoni. 2000. "Deoxyadenosine Analogs Induce Programmed Cell Death in Chronic Lymphocytic Leukemia Cells by Damaging the DNA and by Directly Affecting the Mitochondria." Blood 96 (10): 3537-43.

Giovannetti, Elisa, Mario Del Tacca, Valentina Mey, Niccola Funel, Sara Nannizzi, Sergio Ricci, Cinzia Orlandini, et al. 2006. "Transcription Analysis of Human Equilibrative Nucleoside Transporter-1 Predicts Survival in Pancreas Cancer Patients Treated 
with Gemcitabine." Cancer Research 66 (7): 3928-35. doi:10.1158/00085472.CAN-05-4203.

Gonzalez, Sonia L., Matt Stremlau, Xi He, John R. Basile, and Karl Münger. 2001. "Degradation of the Retinoblastoma Tumor Suppressor by the Human Papillomavirus Type 16 E7 Oncoprotein Is Important for Functional Inactivation and Is Separable from Proteasomal Degradation of E7." Journal of Virology 75 (16): 7583-91. doi:10.1128/JVI.75.16.7583-7591.2001.

Gore, Steven D., Stephen Baylin, Elizabeth Sugar, Hetty Carraway, Carole B. Miller, Michael Carducci, Michael Grever, et al. 2006. "Combined DNA Methyltransferase and Histone Deacetylase Inhibition in the Treatment of Myeloid Neoplasms." Cancer Research 66 (12): 6361-69. doi:10.1158/0008-5472.CAN-06-0080.

Gottifredi, Vanesa, Orit Karni-Schmidt, Sheau-Yann Shieh, and Carol Prives. 2001. "p53 Down-Regulates CHK1 through p21 and the Retinoblastoma Protein." Molecular and Cellular Biology 21 (4): 1066-76. doi:10.1128/MCB.21.4.1066-1076.2001.

Guertin, Amy D., Melissa M. Martin, Brian Roberts, Melissa Hurd, Xianlu Qu, Nathan R. Miselis, Yaping Liu, et al. 2012. "Unique Functions of CHK1 and WEE1 Underlie Synergistic Anti-Tumor Activity upon Pharmacologic Inhibition." Cancer Cell International 12 (1): 45. doi:10.1186/1475-2867-12-45.

Guo, Z, A Kumagai, S X Wang, and W G Dunphy. 2000. "Requirement for Atr in Phosphorylation of Chk1 and Cell Cycle Regulation in Response to DNA Replication Blocks and UV-Damaged DNA in Xenopus Egg Extracts." Genes \& Development 14 (21): 2745-56.

Guzi, Timothy J, Kamil Paruch, Michael P Dwyer, Marc Labroli, Frances Shanahan, Nicole Davis, Lorena Taricani, et al. 2011. "Targeting the Replication Checkpoint Using SCH 900776, a Potent and Functionally Selective CHK1 Inhibitor Identified via High Content Screening." Molecular Cancer Therapeutics 10 (4): 591-602. doi:10.1158/1535-7163.MCT-10-0928.

Hamer, Philip C. De Witt, Shahryar E. Mir, David Noske, Cornelis J. F. Van Noorden, and Tom Würdinger. 2011. "WEE1 Kinase Targeting Combined with DNA-Damaging Cancer Therapy Catalyzes Mitotic Catastrophe." Clinical Cancer Research 17 (13): 4200-4207. doi:10.1158/1078-0432.CCR-10-2537.

Hanada, Katsuhiro, Magda Budzowska, Sally L. Davies, Ellen van Drunen, Hideo Onizawa, H. Berna Beverloo, Alex Maas, Jeroen Essers, lan D. Hickson, and Roland Kanaar. 2007. "The Structure-Specific Endonuclease Mus81 Contributes to Replication Restart by Generating Double-Strand DNA Breaks." Nature Structural \& Molecular Biology 14 (11): 1096-1104. doi:10.1038/nsmb1313.

Hans, F, and S Dimitrov. 2001. "Histone H3 Phosphorylation and Cell Division." Oncogene 20 (24): 3021-27. doi:10.1038/sj.onc.1204326.

Harbour, J. William, and Douglas C. Dean. 2000. "The Rb/E2F Pathway: Expanding Roles and Emerging Paradigms." Genes \& Development 14 (19): 2393-2409. doi:10.1101/gad.813200.

Heinemann, V, L W Hertel, G B Grindey, and W Plunkett. 1988. "Comparison of the Cellular Pharmacokinetics and Toxicity of 2',2'-Difluorodeoxycytidine and 1-BetaD-Arabinofuranosylcytosine." Cancer Research 48 (14): 4024-31.

Heinemann, V, L Schulz, R D Issels, and W Plunkett. 1995. "Gemcitabine: A Modulator of Intracellular Nucleotide and Deoxynucleotide Metabolism." Seminars in Oncology 22 (4 Suppl 11): 11-18.

Heinemann, V, Y Z Xu, S Chubb, A Sen, L W Hertel, G B Grindey, and W Plunkett. 1990. "Inhibition of Ribonucleotide Reduction in CCRF-CEM Cells by 2',2'Difluorodeoxycytidine." Molecular Pharmacology 38 (4): 567-72.

1992. "Cellular Elimination of 2',2'-Difluorodeoxycytidine 5'-Triphosphate: A Mechanism of Self-Potentiation." Cancer Research 52 (3): 533-39.

Hirai, Hiroshi, Yoshikazu Iwasawa, Megumu Okada, Tsuyoshi Arai, Toshihide Nishibata, Makiko Kobayashi, Toshifumi Kimura, et al. 2009. "Small-Molecule Inhibition of Wee1 Kinase by MK-1775 Selectively Sensitizes p53-Deficient Tumor Cells to 
DNA-Damaging Agents." Molecular Cancer Therapeutics 8 (11): 2992-3000. doi:10.1158/1535-7163.MCT-09-0463.

Hirao, Atsushi, Young-Yun Kong, Shuhei Matsuoka, Andrew Wakeham, Jürgen Ruland, Hiroki Yoshida, Dou Liu, Stephen J. Elledge, and Tak W. Mak. 2000. "DNA Damage-Induced Activation of p53 by the Checkpoint Kinase Chk2." Science 287 (5459): 1824-27. doi:10.1126/science.287.5459.1824.

Hollstein, M., D. Sidransky, B. Vogelstein, and C. C. Harris. 1991. "p53 Mutations in Human Cancers." Science 253 (5015): 49-53. doi:10.1126/science.1905840.

Huang, P, S Chubb, L W Hertel, G B Grindey, and W Plunkett. 1991. "Action of 2',2'Difluorodeoxycytidine on DNA Synthesis." Cancer Research 51 (22): 6110-17.

Huang, P, S Chubb, and W Plunkett. 1990. "Termination of DNA Synthesis by 9-Beta-DArabinofuranosyl-2-Fluoroadenine. A Mechanism for Cytotoxicity." The Journal of Biological Chemistry 265 (27): 16617-25.

Jackson, J R, A Gilmartin, C Imburgia, J D Winkler, L A Marshall, and A Roshak. 2000. "An Indolocarbazole Inhibitor of Human Checkpoint Kinase (Chk1) Abrogates Cell Cycle Arrest Caused by DNA Damage." Cancer Research 60 (3): 566-72.

Jazayeri, Ali, Jacob Falck, Claudia Lukas, Jiri Bartek, Graeme C M Smith, Jiri Lukas, and Stephen P Jackson. 2006. "ATM- and Cell Cycle-Dependent Regulation of ATR in Response to DNA Double-Strand Breaks." Nature Cell Biology 8 (1): 37-45. doi:10.1038/ncb1337.

Karnitz, Larry M, Karen S Flatten, Jill M Wagner, David Loegering, Jennifer S Hackbarth, Sonnet J H Arlander, Benjamin T Vroman, et al. 2005. "Gemcitabine-Induced Activation of Checkpoint Signaling Pathways That Affect Tumor Cell Survival." Molecular Pharmacology 68 (6): 1636-44. doi:10.1124/mol.105.012716.

Kasahara, Kousuke, Hidemasa Goto, Masato Enomoto, Yasuko Tomono, Tohru Kiyono, and Masaki Inagaki. 2010. "14-3-3gamma Mediates Cdc25A Proteolysis to Block Premature Mitotic Entry after DNA Damage." The EMBO Journal 29 (16): 280212. doi:10.1038/emboj.2010.157.

Kastan, M B, and D S Lim. 2000. "The Many Substrates and Functions of ATM." Nature Reviews. Molecular Cell Biology 1 (3): 179-86. doi:10.1038/35043058.

Katayama, Kazuhiro, Naoya Fujita, and Takashi Tsuruo. 2005. "Akt/Protein Kinase BDependent Phosphorylation and Inactivation of WEE1Hu Promote Cell Cycle Progression at G2/M Transition." Molecular and Cellular Biology 25 (13): 5725-37. doi:10.1128/MCB.25.13.5725-5737.2005.

Kim, Michael P., and Gary E. Gallick. 2008. "Gemcitabine Resistance in Pancreatic Cancer: Picking the Key Players." Clinical Cancer Research 14 (5): 1284-85. doi:10.1158/1078-0432.CCR-07-2247.

Kim, Seong-Tae, Bo Xu, and Michael B Kastan. 2002. "Involvement of the Cohesin Protein, Smc1, in Atm-Dependent and Independent Responses to DNA Damage." Genes \& Development 16 (5): 560-70. doi:10.1101/gad.970602.

Kitagawa, Risa, Christopher J Bakkenist, Peter J McKinnon, and Michael B Kastan. 2004. "Phosphorylation of SMC1 Is a Critical Downstream Event in the ATM-NBS1BRCA1 Pathway." Genes \& Development 18 (12): 1423-38. doi:10.1101/gad.1200304.

Kousholt, Arne Nedergaard, Kasper Fugger, Saskia Hoffmann, Brian D. Larsen, Tobias Menzel, Alessandro A. Sartori, and Claus Storgaard Sørensen. 2012. "CtIPDependent DNA Resection Is Required for DNA Damage Checkpoint Maintenance but Not Initiation." The Journal of Cell Biology 197 (7): 869-76. doi:10.1083/jcb.201111065.

Kranz, Dominique, and Matthias Dobbelstein. 2006. "Nongenotoxic p53 Activation Protects Cells against S-Phase-Specific Chemotherapy." Cancer Research 66 (21): 10274-80. doi:10.1158/0008-5472.CAN-06-1527.

Kruhlak, Michael J, Arkady Celeste, Graham Dellaire, Oscar Fernandez-Capetillo, Waltraud G Müller, James G McNally, David P Bazett-Jones, and André Nussenzweig. 2006. "Changes in Chromatin Structure and Mobility in Living Cells 
at Sites of DNA Double-Strand Breaks." The Journal of Cell Biology 172 (6): 82334. doi:10.1083/jcb.200510015.

Kufe, D W, P P Major, E M Egan, and G P Beardsley. 1980. "Correlation of Cytotoxicity with Incorporation of Ara-C into DNA." The Journal of Biological Chemistry 255 (19): 8997-8900.

Kumagai, A, and W G Dunphy. 1991. "The cdc25 Protein Controls Tyrosine Dephosphorylation of the cdc2 Protein in a Cell-Free System." Cell 64 (5): 903-14.

Kumagai, Akiko, and William G. Dunphy. 2003. "Repeated Phosphopeptide Motifs in Claspin Mediate the Regulated Binding of Chk1." Nature Cell Biology 5 (2): 16165. doi:10.1038/ncb921.

Kumagai, Akiko, Joon Lee, Hae Yong Yoo, and William G. Dunphy. 2006. "TopBP1 Activates the ATR-ATRIP Complex." Cell 124 (5): 943-55. doi:10.1016/j.cell.2005.12.041.

Lavin, Martin F, and Sergei Kozlov. 2007. "ATM Activation and DNA Damage Response." Cell Cycle (Georgetown, Tex.) 6 (8): 931-42.

Lavin, Martin F. 2008. "Ataxia-Telangiectasia: From a Rare Disorder to a Paradigm for Cell Signalling and Cancer." Nature Reviews Molecular Cell Biology 9 (10): 759_ 69. doi:10.1038/nrm2514.

Lee, Ji-Hoon, and Tanya T. Paull. 2005. "ATM Activation by DNA Double-Strand Breaks Through the Mre11-Rad50-Nbs1 Complex." Science 308 (5721): 551-54. doi:10.1126/science.1108297.

Lee, Joon, Akiko Kumagai, and William G. Dunphy. 2001a. "Positive Regulation of Wee1 by Chk1 and 14-3-3 Proteins." Molecular Biology of the Cell 12 (3): 551-63. http://www.ncbi.nlm.nih.gov/pmc/articles/PMC30963/.

. 2007. "The Rad9-Hus1-Rad1 Checkpoint Clamp Regulates Interaction of TopBP1 with ATR." Journal of Biological Chemistry 282 (38): 28036-44. doi:10.1074/jbc.M704635200.

Lee, Kyung S., Jung-Eun Park, Young Hwi Kang, Wendy Zimmerman, Nak-Kyun Soung, Yeon-Sun Seong, Sahng-June Kwak, and Raymond L. Erikson. 2008. "Mechanisms of Mammalian Polo-like Kinase 1 (Plk1) Localization: Self-versus Non-Self-Priming." Cell Cycle 7 (2): 141-45. doi:10.4161/cc.7.2.5272.

Lee, $\mathrm{M} \mathrm{H}$, and H Y Yang. 2001. "Negative Regulators of Cyclin-Dependent Kinases and Their Roles in Cancers." Cellular and Molecular Life Sciences: CMLS 58 (12-13): 1907-22.

Lee, Tae-Jin, Eun Jung Kim, Shin Kim, Eun Mi Jung, Jong-Wook Park, Seung Hun Jeong, Sang Eun Park, Young Hyun Yoo, and Taeg Kyu Kwon. 2006. "CaspaseDependent and Caspase-Independent Apoptosis Induced by Evodiamine in Human Leukemic U937 Cells." Molecular Cancer Therapeutics 5 (9): 2398-2407. doi:10.1158/1535-7163.MCT-06-0167.

Leeuwen, Ingeborg M. M. van, Maureen Higgins, Johanna Campbell, Christopher J. Brown, Anna R. McCarthy, Lisa Pirrie, Nicholas J. Westwood, and Sonia Laín. 2011. "Mechanism-Specific Signatures for Small-Molecule p53 Activators." Cell Cycle 10 (10): 1590-98. doi:10.4161/cc.10.10.15519.

Leung-Pineda, Van, Christine E. Ryan, and Helen Piwnica-Worms. 2006. "Phosphorylation of Chk1 by ATR Is Antagonized by a Chk1-Regulated Protein Phosphatase 2A Circuit." Molecular and Cellular Biology 26 (20): 7529-38. doi:10.1128/MCB.00447-06.

Levine, A J. 1997. "p53, the Cellular Gatekeeper for Growth and Division." Cell 88 (3): 323-31.

Liu, Q, S Guntuku, X S Cui, S Matsuoka, D Cortez, K Tamai, G Luo, et al. 2000. "Chk1 Is an Essential Kinase That Is Regulated by Atr and Required for the G(2)/M DNA Damage Checkpoint." Genes \& Development 14 (12): 1448-59.

Liu, Shizhou, Bunsyo Shiotani, Mayurika Lahiri, Alexandre Marechal, Alice Tse, Charles Chung Yun Leung, J. N. Mark Glover, Xiaohong H. Yang, and Lee Zou. 2011. "ATR Autophosphorylation as a Molecular Switch for Checkpoint Activation." Molecular Cell 43 (2): 192-202. doi:10.1016/j.molcel.2011.06.019. 
Lopes, M, C Cotta-Ramusino, A Pellicioli, G Liberi, P Plevani, M Muzi-Falconi, C S Newlon, and M Foiani. 2001. "The DNA Replication Checkpoint Response Stabilizes Stalled Replication Forks." Nature 412 (6846): 557-61. doi:10.1038/35087613.

Lucca, Chiara, Fabio Vanoli, Cecilia Cotta-Ramusino, Achille Pellicioli, Giordano Liberi, James Haber, and Marco Foiani. 2004. "Checkpoint-Mediated Control of Replisome-Fork Association and Signalling in Response to Replication Pausing." Oncogene 23 (6): 1206-13. doi:10.1038/sj.onc.1207199.

Lukas, Claudia, Jacob Falck, Jirina Bartkova, Jiri Bartek, and Jiri Lukas. 2003. "Distinct Spatiotemporal Dynamics of Mammalian Checkpoint Regulators Induced by DNA Damage." Nature Cell Biology 5 (3): 255-60. doi:10.1038/ncb945.

Lundberg, Ante S., and Robert A. Weinberg. 1998. "Functional Inactivation of the Retinoblastoma Protein Requires Sequential Modification by at Least Two Distinct Cyclin-Cdk Complexes." Molecular and Cellular Biology 18 (2): 753-61.

Ma, Cynthia X., Matthew J. C. Ellis, Gina R. Petroni, Zhanfang Guo, Shi-rong Cai, Christine E. Ryan, A. Craig Lockhart, et al. 2013. "A Phase II Study of UCN-01 in Combination with Irinotecan in Patients with Metastatic Triple Negative Breast Cancer." Breast Cancer Research and Treatment 137 (2): 483-92. doi:10.1007/s10549-012-2378-9.

Macůrek, Libor, Arne Lindqvist, Dan Lim, Michael A. Lampson, Rob Klompmaker, Raimundo Freire, Christophe Clouin, Stephen S. Taylor, Michael B. Yaffe, and René H. Medema. 2008. "Polo-like Kinase-1 Is Activated by Aurora A to Promote Checkpoint Recovery." Nature 455 (7209): 119-23. doi:10.1038/nature07185.

Mahon, Patrick C, Patrick Baril, Vipul Bhakta, Claude Chelala, Krishna Caulee, Tomohiko Harada, and Nicholas R Lemoine. 2007. "S100A4 Contributes to the Suppression of BNIP3 Expression, Chemoresistance, and Inhibition of Apoptosis in Pancreatic Cancer." Cancer Research 67 (14): 6786-95. doi:10.1158/0008-5472.CAN-070440.

Mailand, N, J Falck, C Lukas, R G Syljuâsen, M Welcker, J Bartek, and J Lukas. 2000. "Rapid Destruction of Human Cdc25A in Response to DNA Damage." Science (New York, N.Y.) 288 (5470): 1425-29.

Mailand, Niels, Simon Bekker-Jensen, Jiri Bartek, and Jiri Lukas. 2006a. "Destruction of Claspin by SCF 3 TrCP Restrains Chk1 Activation and Facilitates Recovery from Genotoxic Stress." Molecular Cell 23 (3): 307-18. doi:10.1016/j.molcel.2006.06.016.

Mailand, Niels, Simon Bekker-Jensen, Helene Faustrup, Fredrik Melander, Jiri Bartek, Claudia Lukas, and Jiri Lukas. 2007. "RNF8 Ubiquitylates Histones at DNA Double-Strand Breaks and Promotes Assembly of Repair Proteins." Cell 131 (5): 887-900. doi:10.1016/j.cell.2007.09.040.

Malhotra, Vikas, and Michael C. Perry. 2003. "Classical Chemotherapy: Mechanisms, Toxicities and the Therapeutc Window." Cancer Biology \& Therapy 2 (0): 1-3.

Malumbres, Marcos, and Mariano Barbacid. 2005. "Mammalian Cyclin-Dependent Kinases." Trends in Biochemical Sciences 30 (11): 630-41. doi:10.1016/j.tibs.2005.09.005.

Malumbres, Marcos, Edward Harlow, Tim Hunt, Tony Hunter, Jill M. Lahti, Gerard Manning, David O. Morgan, Li-Huei Tsai, and Debra J. Wolgemuth. 2009. "CyclinDependent Kinases: A Family Portrait." Nature Cell Biology 11 (11): 1275-76. doi:10.1038/ncb1109-1275.

Mamely, Ivan, Marcel Atm van Vugt, Veronique A J Smits, Jennifer I Semple, Bennie Lemmens, Anastassis Perrakis, René H Medema, and Raimundo Freire. 2006. "Polo-like Kinase-1 Controls Proteasome-Dependent Degradation of Claspin during Checkpoint Recovery." Current Biology: CB 16 (19): 1950-55. doi:10.1016/j.cub.2006.08.026.

Mannino, Jennifer L., Wan-Ju Kim, Meredith Wernick, Son V. Nguyen, Ray Braquet, Aaron W. Adamson, Zhining Den, Mark A. Batzer, Colin C. Collins, and Kevin D. Brown. 2001. "Evidence for Alternate Splicing within the mRNA Transcript 
Encoding the DNA Damage Response Kinase ATR." Gene 272 (1-2): 35-43. doi:10.1016/S0378-1119(01)00543-1.

Martín, Yusé, Raquel Domínguez-Kelly, and Raimundo Freire. 2011. "Novel Insights into Maintaining Genomic Integrity: Wee1 Regulating Mus81/Eme1." Cell Division 6 (1): 21. doi:10.1186/1747-1028-6-21.

Maya, Ruth, Moshe Balass, Seong-Tae Kim, Dganit Shkedy, Juan-Fernando Martinez Leal, Ohad Shifman, Miri Moas, et al. 2001. "ATM-Dependent Phosphorylation of Mdm2 on Serine 395: Role in p53 Activation by DNA Damage." Genes \& Development 15 (9): 1067-77. doi:10.1101/gad.886901.

McCubrey, James A., Linda S. Steelman, William H. Chappell, Stephen L. Abrams, Ellis W. T. Wong, Fumin Chang, Brian Lehmann, et al. 2007. "Roles of the Raf/MEK/ERK Pathway in Cell Growth, Malignant Transformation and Drug Resistance." Biochimica et Biophysica Acta (BBA) - Molecular Cell Research, Mitogen-Activated Protein Kinases: New Insights on Regulation, Function and Role in Human Disease, 1773 (8): 1263-84. doi:10.1016/j.bbamcr.2006.10.001.

McKnight, Josephine A. 2003. "Principles of Chemotherapy." Clinical Techniques in Small Animal Practice, Medical Oncology, 18 (2): 67-72. doi:10.1053/svms.2003.36617.

McNeely, Samuel, Chiara Conti, Tahir Sheikh, Himali Patel, Sonya Zabludoff, Yves G. Pommier, Gary K. Schwartz, and Archie Tse. 2010. "Chk1 Inhibition after Replicative Stress Activates a Double Strand Break Response Mediated by ATM and DNA-Dependent Protein Kinase." Cell Cycle 9 (5): 995-1004. doi:10.4161/cc.9.5.10935.

Medhurst, Annette L., Daniël O. Warmerdam, Ildem Akerman, Edward H. Verwayen, Roland Kanaar, Veronique A. J. Smits, and Nicholas D. Lakin. 2008. "ATR and Rad17 Collaborate in Modulating Rad9 Localisation at Sites of DNA Damage." Journal of Cell Science 121 (23): 3933-40. doi:10.1242/jcs.033688.

Meijer, L., A. Borgne, O. Mulner, J. P. Chong, J. J. Blow, N. Inagaki, M. Inagaki, J. G. Delcros, and J. P. Moulinoux. 1997. "Biochemical and Cellular Effects of Roscovitine, a Potent and Selective Inhibitor of the Cyclin-Dependent Kinases cdc2, cdk2 and cdk5." European Journal of Biochemistry / FEBS 243 (1-2): 52736.

Melo, Justine A., Jen Cohen, and David P. Toczyski. 2001. "Two Checkpoint Complexes Are Independently Recruited to Sites of DNA Damage in Vivo." Genes \& Development 15 (21): 2809-21. doi:10.1101/gad.903501.

Miyachi, Mitsuru, Naoki Kakazu, Shigeki Yagyu, Yoshiki Katsumi, Satoko Tsubai-Shimizu, Ken Kikuchi, Kunihiko Tsuchiya, Tomoko lehara, and Hajime Hosoi. 2009. "Restoration of p53 Pathway by Nutlin-3 Induces Cell Cycle Arrest and Apoptosis in Human Rhabdomyosarcoma Cells." Clinical Cancer Research 15 (12): 407784. doi:10.1158/1078-0432.CCR-08-2955.

Moore, M J, J Hamm, J Dancey, P D Eisenberg, M Dagenais, A Fields, K Hagan, et al. 2003. "Comparison of Gemcitabine versus the Matrix Metalloproteinase Inhibitor BAY 12-9566 in Patients with Advanced or Metastatic Adenocarcinoma of the Pancreas: A Phase III Trial of the National Cancer Institute of Canada Clinical Trials Group." Journal of Clinical Oncology: Official Journal of the American Society of Clinical Oncology 21 (17): 3296-3302. doi:10.1200/JCO.2003.02.098.

Morgan, David O. 1995. "Principles of CDK Regulation." Nature 374 (6518): 131-34. doi:10.1038/374131a0.

Mukherjee, Bipasha, Chase Kessinger, Junya Kobayashi, Benjamin P C Chen, David J Chen, Aloke Chatterjee, and Sandeep Burma. 2006. "DNA-PK Phosphorylates Histone H2AX during Apoptotic DNA Fragmentation in Mammalian Cells." DNA Repair 5 (5): 575-90. doi:10.1016/j.dnarep.2006.01.011.

Murakami, Monica S., Terry D. Copeland, and George F. Vande Woude. 1999. "Mos Positively Regulates Xe-Wee1 to Lengthen the First Mitotic Cell Cycle of Xenopus." Genes \& Development 13 (5): 620-31.

Murfuni, Ivana, Giorgia Basile, Shyamal Subramanyam, Eva Malacaria, Margherita Bignami, Maria Spies, Annapaola Franchitto, and Pietro Pichierri. 2013. "Survival 
of the Replication Checkpoint Deficient Cells Requires MUS81-RAD52 Function." PLoS Genet 9 (10): e1003910. doi:10.1371/journal.pgen.1003910.

Myers, Jeremy S, and David Cortez. 2006. "Rapid Activation of ATR by Ionizing Radiation Requires ATM and Mre11." The Journal of Biological Chemistry 281 (14): 934650. doi:10.1074/jbc.M513265200.

Nakano, Y, S Tanno, K Koizumi, T Nishikawa, K Nakamura, M Minoguchi, T Izawa, Y Mizukami, T Okumura, and Y Kohgo. 2007. "Gemcitabine Chemoresistance and Molecular Markers Associated with Gemcitabine Transport and Metabolism in Human Pancreatic Cancer Cells." British Journal of Cancer 96 (3): 457-63. doi:10.1038/sj.bjc.6603559.

Nam, E. A., R. Zhao, G. G. Glick, C. E. Bansbach, D. B. Friedman, and D. Cortez. 2011. "Thr-1989 Phosphorylation Is a Marker of Active Ataxia Telangiectasia-Mutated and Rad3-Related (ATR) Kinase." Journal of Biological Chemistry 286 (33): 28707-14. doi:10.1074/jbc.M111.248914.

Ng, Sylvia S. W., Ming-Sound Tsao, Sue Chow, and David W. Hedley. 2000. "Inhibition of Phosphatidylinositide 3-Kinase Enhances Gemcitabine-Induced Apoptosis in Human Pancreatic Cancer Cells." Cancer Research 60 (19): 5451-55.

Ng, Sylvia S. W., Ming-Sound Tsao, Trudey Nicklee, and David W. Hedley. 2001. "Wortmannin Inhibits PKB/Akt Phosphorylation and Promotes Gemcitabine Antitumor Activity in Orthotopic Human Pancreatic Cancer Xenografts in Immunodeficient Mice." Clinical Cancer Research 7 (10): 3269-75.

Nigro, Janice M., Suzanne J. Baker, Antonette C. Preisinger, J. Milburn Jessup, Richard Hosteller, Karen Cleary, Sandra H. Signer, et al. 1989. "Mutations in the p53 Gene Occur in Diverse Human Tumour Types." Nature 342 (6250): 705-8. doi:10.1038/342705a0.

O'Connell, Matthew J., Jeanette M. Raleigh, Heather M. Verkade, and Paul Nurse. 1997. "Chk1 Is a wee1 Kinase in the G2 DNA Damage Checkpoint Inhibiting cdc2 by Y15 Phosphorylation." The EMBO Journal 16 (3): 545-54. doi:10.1093/emboj/16.3.545.

Oliner, J D, J A Pietenpol, S Thiagalingam, J Gyuris, K W Kinzler, and B Vogelstein. 1993. "Oncoprotein MDM2 Conceals the Activation Domain of Tumour Suppressor p53." Nature 362 (6423): 857-60. doi:10.1038/362857a0.

Olmos, David, Douglas Barker, Rohini Sharma, Andre T. Brunetto, Timothy A. Yap, Anne B. Taegtmeyer, Jorge Barriuso, et al. 2011. "Phase I Study of GSK461364, a Specific and Competitive Polo-like Kinase 1 Inhibitor, in Patients with Advanced Solid Malignancies." Clinical Cancer Research: An Official Journal of the American Association for Cancer Research 17 (10): 3420-30. doi:10.1158/1078-0432.CCR10-2946.

Osman, Fekret, and Matthew C. Whitby. 2007. "Exploring the Roles of Mus81Eme1/Mms4 at Perturbed Replication Forks." DNA Repair, Replication Fork Repair Processes, 6 (7): 1004-17. doi:10.1016/j.dnarep.2007.02.019.

Parker, L L, and H Piwnica-Worms. 1992. "Inactivation of the p34cdc2-Cyclin B Complex by the Human WEE1 Tyrosine Kinase." Science (New York, N.Y.) 257 (5078): 1955-57.

Paull, T T, E P Rogakou, V Yamazaki, C U Kirchgessner, M Gellert, and W M Bonner. 2000. "A Critical Role for Histone H2AX in Recruitment of Repair Factors to Nuclear Foci after DNA Damage." Current Biology: CB 10 (15): 886-95.

Paulsen, Renee D., and Karlene A. Cimprich. 2007. "The ATR Pathway: Fine-Tuning the Fork." DNA Repair, Replication Fork Repair Processes, 6 (7): 953-66. doi:10.1016/j.dnarep.2007.02.015.

Peng, C Y, P R Graves, R S Thoma, Z Wu, A S Shaw, and H Piwnica-Worms. 1997. "Mitotic and G2 Checkpoint Control: Regulation of 14-3-3 Protein Binding by Phosphorylation of Cdc25C on Serine-216." Science (New York, N.Y.) 277 (5331): 1501-5.

Peng, Cheng-Yuan, Paul R. Graves, Richard S. Thoma, Zhiqi Wu, Andrey S. Shaw, and Helen Piwnica-Worms. 1997. "Mitotic and G2 Checkpoint Control: Regulation of 
14-3-3 Protein Binding by Phosphorylation of Cdc25C on Serine-216." Science 277 (5331): 1501-5. doi:10.1126/science.277.5331.1501.

Perez, Raymond P., Lionel D. Lewis, Andrew P. Beelen, Anthony J. Olszanski, Nicholas Johnston, C. Harker Rhodes, Bernard Beaulieu, Marc S. Ernstoff, and Alan Eastman. 2006. "Modulation of Cell Cycle Progression in Human Tumors: A Pharmacokinetic and Tumor Molecular Pharmacodynamic Study of Cisplatin Plus the Chk1 Inhibitor UCN-01 (NSC 638850)." Clinical Cancer Research 12 (23): 7079-85. doi:10.1158/1078-0432.CCR-06-0197.

Peschiaroli, Angelo, N. Valerio Dorrello, Daniele Guardavaccaro, Monica Venere, Thanos Halazonetis, Nicholas E. Sherman, and Michele Pagano. 2006. "SCF $\beta T r C P$ Mediated Degradation of Claspin Regulates Recovery from the DNA Replication Checkpoint Response." Molecular Cell 23 (3): 319-29. doi:10.1016/j.molcel.2006.06.013.

Plunkett, W, P Huang, Y Z Xu, V Heinemann, R Grunewald, and V Gandhi. 1995. "Gemcitabine: Metabolism, Mechanisms of Action, and Self-Potentiation." Seminars in Oncology 22 (4 Suppl 11): 3-10.

Polager, Shirley, and Doron Ginsberg. 2009. "p53 and E2f: Partners in Life and Death." Nature Reviews Cancer 9 (10): 738-48. doi:10.1038/nrc2718.

Pourquier, Philippe, Christopher Gioffre, Glenda Kohlhagen, Yoshimasa Urasaki, François Goldwasser, Lary W. Hertel, Shuyuan Yu, Richard T. Pon, William H. Gmeiner, and Yves Pommier. 2002. "Gemcitabine (2',2'-Difluoro-2'-Deoxycytidine), an Antimetabolite That Poisons Topoisomerase I." Clinical Cancer Research 8 (8): 2499-2504.

Prevo, Remko, Emmanouil Fokas, Philip M Reaper, Peter A Charlton, John R Pollard, W Gillies McKenna, Ruth J Muschel, and Thomas B Brunner. 2012a. "The Novel ATR Inhibitor VE-821 Increases Sensitivity of Pancreatic Cancer Cells to Radiation and Chemotherapy." Cancer Biology \& Therapy 13 (11): 1072-81. doi:10.4161/cbt.21093.

Rajeshkumar, N. V., E. De Oliveira, N. Ottenhof, J. Watters, D. Brooks, T. Demuth, S. D. Shumway, et al. 2011. "MK-1775, a Potent Wee1 Inhibitor, Synergizes with Gemcitabine to Achieve Tumor Regressions, Selectively in p53-Deficient Pancreatic Cancer Xenografts." Clinical Cancer Research 17 (9): 2799-2806. doi:10.1158/1078-0432.CCR-10-2580.

Rajeshkumar, N. V., Elizabeth De Oliveira, Niki Ottenhof, James Watters, David Brooks, Tim Demuth, Stuart D. Shumway, et al. 2011. "MK-1775, a Potent Wee1 Inhibitor, Synergizes with Gemcitabine to Achieve Tumor Regressions, Selectively in p53Deficient Pancreatic Cancer Xenografts." Clinical Cancer Research 17 (9): 2799_ 2806. doi:10.1158/1078-0432.CCR-10-2580.

Reaper, Philip M., Matthew R. Griffiths, Joanna M. Long, Jean-Damien Charrier, Somhairle MacCormick, Peter A. Charlton, Julian M. C. Golec, and John R. Pollard. 2011. "Selective Killing of ATM- or p53-Deficient Cancer Cells through Inhibition of ATR." Nature Chemical Biology 7 (7): 428-30. doi:10.1038/nchembio.573.

Reitsema, Tarren, Dmitry Klokov, Judit P Banáth, and Peggy L Olive. 2005. "DNA-PK Is Responsible for Enhanced Phosphorylation of Histone H2AX under Hypertonic Conditions." DNA Repair 4 (10): 1172-81. doi:10.1016/j.dnarep.2005.06.005.

Ren, Bing, Hieu Cam, Yasuhiko Takahashi, Thomas Volkert, Jolyon Terragni, Richard A. Young, and Brian David Dynlacht. 2002. "E2F Integrates Cell Cycle Progression with DNA Repair, Replication, and G2/M Checkpoints." Genes \& Development 16 (2): 245-56. doi:10.1101/gad.949802.

Riedl, Stefan J., and Guy S. Salvesen. 2007. "The Apoptosome: Signalling Platform of Cell Death." Nature Reviews Molecular Cell Biology 8 (5): 405-13. doi:10.1038/nrm2153.

Rogakou, E P, C Boon, C Redon, and W M Bonner. 1999. "Megabase Chromatin Domains Involved in DNA Double-Strand Breaks in Vivo." The Journal of Cell Biology 146 (5): 905-16. 
Rogakou, E P, D R Pilch, A H Orr, V S Ivanova, and W M Bonner. 1998. "DNA DoubleStranded Breaks Induce Histone H2AX Phosphorylation on Serine 139." The Journal of Biological Chemistry 273 (10): 5858-68.

Rogakou, Emmy P., Duane R. Pilch, Ann H. Orr, Vessela S. Ivanova, and William M. Bonner. 1998. "DNA Double-Stranded Breaks Induce Histone H2AX Phosphorylation on Serine 139." Journal of Biological Chemistry 273 (10): 585868. http://www.jbc.org/content/273/10/5858.

Rothblum-Oviatt, Cynthia J., Christine E. Ryan, and Helen Piwnica-Worms. 2001a. "14-33 Binding Regulates Catalytic Activity of Human Wee1 Kinase." Cell Growth Differentiation 12 (12): 581-89. http://cgd.aacrjournals.org/cgi/content/abstract/12/12/581.

Ryan, K M, A C Phillips, and K H Vousden. 2001. "Regulation and Function of the p53 Tumor Suppressor Protein." Current Opinion in Cell Biology 13 (3): 332-37.

S?rensen, Claus Storgaard, and Randi G. Syljuasen. 2012. "Safeguarding Genome Integrity: The Checkpoint Kinases ATR, CHK1 and WEE1 Restrain CDK Activity during Normal DNA Replication." Nucleic Acids Research 40 (2): 477-86. doi:10.1093/nar/gkr697.

Sampath, Deepa, Zheng Shi, and William Plunkett. 2002. "Inhibition of Cyclin-Dependent Kinase 2 by the Chk1-Cdc25A Pathway during the S-Phase Checkpoint Activated by Fludarabine: Dysregulation by 7-Hydroxystaurosporine." Molecular Pharmacology 62 (3): 680-88.

Sanchez, Y, C Wong, R S Thoma, R Richman, Z Wu, H Piwnica-Worms, and S J Elledge. 1997. "Conservation of the Chk1 Checkpoint Pathway in Mammals: Linkage of DNA Damage to Cdk Regulation through Cdc25." Science (New York, N.Y.) 277 (5331): 1497-1501.

Sandler, A B, J Nemunaitis, C Denham, J von Pawel, Y Cormier, U Gatzemeier, K Mattson, et al. 2000. "Phase III Trial of Gemcitabine plus Cisplatin versus Cisplatin Alone in Patients with Locally Advanced or Metastatic Non-Small-Cell Lung Cancer." Journal of Clinical Oncology: Official Journal of the American Society of Clinical Oncology 18 (1): 122-30.

Santoro, Maxine F., Robert R. Annand, Molly M. Robertson, Yun-Wen Peng, Matthew J. Brady, John A. Mankovich, Maria C. Hackett, et al. 1998. "Regulation of Protein Phosphatase 2A Activity by Caspase-3 during Apoptosis." Journal of Biological Chemistry 273 (21): 13119-28.

Sartori, Alessandro A, and Martin Steger. 2013. "Prolyl Isomerization." Cell Cycle 12 (17): 2717-18. doi:10.4161/cc.26077.

Sartori, Alessandro A., Claudia Lukas, Julia Coates, Martin Mistrik, Shuang Fu, Jiri Bartek, Richard Baer, Jiri Lukas, and Stephen P. Jackson. 2007. "Human CtIP Promotes DNA End Resection." Nature 450 (7169): 509-14. doi:10.1038/nature06337.

Schumacher, Guido, Masafumi Kataoka, Jack A. Roth, and Tapas Mukhopadhyay. 1999. "Potent Antitumor Activity of 2-Methoxyestradiol in Human Pancreatic Cancer Cell Lines." Clinical Cancer Research 5 (3): 493-99.

Seki, Akiko, Judith A. Coppinger, Chang-Young Jang, John R. Yates, and Guowei Fang. 2008. "Bora and the Kinase Aurora A Cooperatively Activate the Kinase Plk1 and Control Mitotic Entry." Science 320 (5883): 1655-58. doi:10.1126/science.1157425.

Shao, J., B. Zhou, Bernard Chu, and Y. Yen. 2006. "Ribonucleotide Reductase Inhibitors and Future Drug Design." Current Cancer Drug Targets 6 (5): 409-31. doi:10.2174/156800906777723949.

Shi, Z, A Azuma, D Sampath, Y X Li, P Huang, and W Plunkett. 2001. "S-Phase Arrest by Nucleoside Analogues and Abrogation of Survival without Cell Cycle Progression by 7-Hydroxystaurosporine." Cancer Research 61 (3): 1065-72.

Shieh, S Y, J Ahn, K Tamai, Y Taya, and C Prives. 2000. "The Human Homologs of Checkpoint Kinases Chk1 and Cds1 (Chk2) Phosphorylate p53 at Multiple DNA Damage-Inducible Sites." Genes \& Development 14 (3): 289-300. 
Shieh, Sheau-Yann, Jinwoo Ahn, Katsuyuki Tamai, Yoichi Taya, and Carol Prives. 2000. "The Human Homologs of Checkpoint Kinases Chk1 and Cds1 (Chk2) Phosphorylate p53 at Multiple DNA Damage-Inducible Sites." Genes \& Development 14 (3):

http://www.ncbi.nlm.nih.gov/pmc/articles/PMC316358/.

Shiloh, Y. 2001. "ATM and ATR: Networking Cellular Responses to DNA Damage." Current Opinion in Genetics \& Development 11 (1): 71-77.

Shiloh, Yosef. 2003. "ATM and Related Protein Kinases: Safeguarding Genome Integrity." Nature Reviews. Cancer 3 (3): 155-68. doi:10.1038/nrc1011.

Simpson-Lavy, Kobi J., and Michael Brandeis. 2011. "Phosphorylation of Cdc5 Regulates Its Accumulation." Cell Division 6 (1): 23. doi:10.1186/1747-1028-6-23.

Smits, Veronique A J, Philip M Reaper, and Stephen P Jackson. 2006. "Rapid PIKKDependent Release of Chk1 from Chromatin Promotes the DNA-Damage Checkpoint Response." Current Biology: CB $16 \quad$ (2): 150-59. doi:10.1016/j.cub.2005.11.066.

Smits, Veronique A. J., Daniel O. Warmerdam, Yuse Martin, and Raimundo Freire. 2010. "Mechanisms of ATR-Mediated Checkpoint Signalling." Frontiers in Bioscience (Landmark Edition) 15: 840-53.

Sogo, José M, Massimo Lopes, and Marco Foiani. 2002. "Fork Reversal and SsDNA Accumulation at Stalled Replication Forks Owing to Checkpoint Defects." Science (New York, N.Y.) 297 (5581): 599-602. doi:10.1126/science.1074023.

Speroni, Juliana, María Belén Federico, Sabrina F. Mansilla, Gastón Soria, and Vanesa Gottifredi. 2012. "Kinase-Independent Function of Checkpoint Kinase 1 (Chk1) in the Replication of Damaged DNA." Proceedings of the National Academy of Sciences 109 (19): 7344-49. doi:10.1073/pnas.1116345109.

Stevens, Craig, and Nicholas B. La Thangue. 2004. "The Emerging Role of E2F-1 in the DNA Damage Response and Checkpoint Control." DNA Repair, BRIDGE OVER BROKEN ENDS - The Cellular Response to DNA Breaks in Health and Disease, 3 (8-9): 1071-79. doi:10.1016/j.dnarep.2004.03.034.

Stevens, Craig, Linda Smith, and Nicholas B La Thangue. 2003. "Chk2 Activates E2F-1 in Response to DNA Damage." Nature Cell Biology 5 (5): 401-9. doi:10.1038/ncb974.

Strebhardt, Klaus, and Axel Ullrich. 2006. "Targeting Polo-like Kinase 1 for Cancer Therapy." Nature Reviews Cancer 6 (4): 321-30. doi:10.1038/nrc1841.

Stresemann, Carlo, and Frank Lyko. 2008. "Modes of Action of the DNA Methyltransferase Inhibitors Azacytidine and Decitabine." International Journal of Cancer. Journal International Du Cancer 123 (1): 8-13. doi:10.1002/ijc.23607.

Sugawara, N., and J. E. Haber. 1992. "Characterization of Double-Strand Break-Induced Recombination: Homology Requirements and Single-Stranded DNA Formation." Molecular and Cellular Biology 12 (2): 563-75. doi:10.1128/MCB.12.2.563.

Takaki, Tohru, Kristina Trenz, Vincenzo Costanzo, and Mark Petronczki. 2008. "Polo-like Kinase 1 Reaches beyond Mitosis--Cytokinesis, DNA Damage Response, and Development." Current Opinion in Cell Biology 20 (6): 650-60. doi:10.1016/j.ceb.2008.10.005.

Taya, Yoichi. 1997. "RB Kinases and RB-Binding Proteins: New Points of View." Trends in Biochemical Sciences 22 (1): 14-17. doi:10.1016/S0968-0004(96)10070-0.

Terrano, David T, Meenakshi Upreti, and Timothy C Chambers. 2010. "Cyclin-Dependent Kinase 1-Mediated $\mathrm{Bcl}-\mathrm{xL} / \mathrm{Bcl}-2$ Phosphorylation Acts as a Functional Link Coupling Mitotic Arrest and Apoptosis." Molecular and Cellular Biology 30 (3): 640-56. doi:10.1128/MCB.00882-09.

Tibbetts, R S, K M Brumbaugh, J M Williams, J N Sarkaria, W A Cliby, S Y Shieh, Y Taya, C Prives, and R T Abraham. 1999. "A Role for ATR in the DNA Damage-Induced Phosphorylation of p53." Genes \& Development 13 (2): 152-57.

Tse, Archie N., Richard Carvajal, and Gary K. Schwartz. 2007. "Targeting Checkpoint Kinase 1 in Cancer Therapeutics." Clinical Cancer Research 13 (7): 1955-60. doi:10.1158/1078-0432.CCR-06-2793. 
Tsuta, Koji, Diane C. Liu, Neda Kalhor, Ignacio I. Wistuba, and Cesar A. Moran. 2011. "Using the Mitosis-Specific Marker Anti-Phosphohistone H3 to Assess Mitosis in Pulmonary Neuroendocrine Carcinomas." American Journal of Clinical Pathology 136 (2): 252-59. doi:10.1309/AJCPDXFOPXGEFORP.

Tsvetkov, Lyuben, and David F. Stern. 2005. "Phosphorylation of Plk1 at S137 and T210 Is Inhibited in Response to DNA Damage." Cell Cycle (Georgetown, Tex.) 4 (1): 166-71.

Van der Donk, Wilfred A., Guixue Yu, Lucy Pérez, Raylene J. Sanchez, JoAnne Stubbe, Vicente Samano, and Morris J. Robins. 1998. "Detection of a New SubstrateDerived Radical during Inactivation of Ribonucleotide Reductase from Escherichia Coli by Gemcitabine 5'-Diphosphate†." Biochemistry 37 (18): 6419-26. doi:10.1021/bi9729357.

Van Vugt, Marcel A. T. M., Alexandra Brás, and René H. Medema. 2004. "Polo-like Kinase-1 Controls Recovery from a G2 DNA Damage-Induced Arrest in Mammalian Cells." Molecular Cell 15 (5): 799-811. doi:10.1016/j.molcel.2004.07.015.

Vassilev, Lyubomir T., Christian Tovar, Shaoqing Chen, Dejan Knezevic, Xiaolan Zhao, Hongmao Sun, David C. Heimbrook, and Li Chen. 2006. "Selective SmallMolecule Inhibitor Reveals Critical Mitotic Functions of Human CDK1." Proceedings of the National Academy of Sciences of the United States of America 103 (28): 10660-65. doi:10.1073/pnas.0600447103.

Vitale, Ilio, Lorenzo Galluzzi, Maria Castedo, and Guido Kroemer. 2011. "Mitotic Catastrophe: A Mechanism for Avoiding Genomic Instability." Nature Reviews Molecular Cell Biology 12 (6): 385-92. doi:10.1038/nrm3115.

Vogel, Celia, Anne Kienitz, Irmgard Hofmann, Rolf Müller, and Holger Bastians. 2004. "Crosstalk of the Mitotic Spindle Assembly Checkpoint with p53 to Prevent Polyploidy." Oncogene 23 (41): 6845-53. doi:10.1038/sj.onc.1207860.

Vogelstein, B, D Lane, and A J Levine. 2000. "Surfing the p53 Network." Nature 408 (6810): 307-10. doi:10.1038/35042675.

Vogelstein, Bert, David Lane, and Arnold J. Levine. 2000. "Surfing the p53 Network." Nature 408 (6810): 307-10. doi:10.1038/35042675.

Von der Maase, H, S W Hansen, J T Roberts, L Dogliotti, T Oliver, M J Moore, I Bodrogi, et al. 2000. "Gemcitabine and Cisplatin versus Methotrexate, Vinblastine, Doxorubicin, and Cisplatin in Advanced or Metastatic Bladder Cancer: Results of a Large, Randomized, Multinational, Multicenter, Phase III Study." Journal of Clinical Oncology: Official Journal of the American Society of Clinical Oncology 18 (17): 3068-77.

Voutsadakis, Ioannis A. 2011. "Molecular Predictors of Gemcitabine Response in Pancreatic Cancer." World Journal of Gastrointestinal Oncology 3 (11): 153-64. doi:10.4251/wjgo.v3.i11.153.

Wang, Jun, Gregory J. S. Lohman, and JoAnne Stubbe. 2007. "Enhanced Subunit Interactions with Gemcitabine-5'-Diphosphate Inhibit Ribonucleotide Reductases." Proceedings of the National Academy of Sciences 104 (36): 14324-29. doi:10.1073/pnas.0706803104.

Wang, Xiaofei, Clare H. McGowan, Ming Zhao, Liusheng He, Jocelyn S. Downey, Colleen Fearns, Yibin Wang, Shi Huang, and Jiahuai Han. 2000. "Involvement of the MKK6-p38? Cascade in ?-Radiation-Induced Cell Cycle Arrest." Molecular and Cellular Biology 20 (13): 4543-52.

Wang, Y, C Jacobs, K E Hook, H Duan, R N Booher, and Y Sun. 2000. "Binding of 14-33beta to the Carboxyl Terminus of Wee1 Increases Wee1 Stability, Kinase Activity, and G2-M Cell Population." Cell Growth \& Differentiation: The Molecular Biology Journal of the American Association for Cancer Research 11 (4): 211-19.

Watanabe, N, M Broome, and T Hunter. 1995. "Regulation of the Human WEE1Hu CDK Tyrosine 15-Kinase during the Cell Cycle." The EMBO Journal 14 (9): 1878-91.

Watanabe, Nobumoto, Harumi Arai, Yoshifumi Nishihara, Makoto Taniguchi, Naoko Watanabe, Tony Hunter, and Hiroyuki Osada. 2004. "M-Phase Kinases Induce 
Phospho-Dependent Ubiquitination of Somatic Wee1 by SCF $\beta$-TrCP." Proceedings of the National Academy of Sciences of the United States of America 101 (13): 4419-24. doi:10.1073/pnas.0307700101.

Wiest, Tina, Elisabeth Schwarz, Christel Enders, Christa Flechtenmacher, and Franz $X$ Bosch. 2002. "Involvement of Intact HPV16 E6/E7 Gene Expression in Head and Neck Cancers with Unaltered p53 Status and Perturbed pRb Cell Cycle Control." Oncogene 21 (10): 1510-17. doi:10.1038/sj.onc.1205214.

Wu, C L, S D Kirley, H Xiao, Y Chuang, D C Chung, and L R Zukerberg. 2001. "Cables Enhances cdk2 Tyrosine 15 Phosphorylation by Wee1, Inhibits Cell Growth, and Is Lost in Many Human Colon and Squamous Cancers." Cancer Research 61 (19): 7325-32.

Wu, Chuan Fen, Ruoning Wang, Qianjin Liang, Jianjiao Liang, Wenke Li, Sung Yun Jung, Jun Qin, Sue-Hwa Lin, and Jian Kuang. 2010. "Dissecting the M Phase-specific Phosphorylation of Serine-Proline or Threonine-Proline Motifs." Molecular Biology of the Cell 21 (9): 1470-81. doi:10.1091/mbc.E09-06-0486.

Xiao, Andrew, Hua Wu, Pier Paolo Pandolfi, David N Louis, and Terry Van Dyke. 2002. "Astrocyte Inactivation of the pRb Pathway Predisposes Mice to Malignant Astrocytoma Development That Is Accelerated by PTEN Mutation." Cancer Cell 1 (2): 157-68. doi:10.1016/S1535-6108(02)00029-6.

Yamauchi, T, B J Nowak, M J Keating, and W Plunkett. 2001. "DNA Repair Initiated in Chronic Lymphocytic Leukemia Lymphocytes by 4-Hydroperoxycyclophosphamide Is Inhibited by Fludarabine and Clofarabine." Clinical Cancer Research: An Official Journal of the American Association for Cancer Research 7 (11): 3580-89.

Yazdi, Parvin T, Yi Wang, Song Zhao, Nimitt Patel, Eva Y-H P Lee, and Jun Qin. 2002. "SMC1 Is a Downstream Effector in the ATM/NBS1 Branch of the Human S-Phase Checkpoint." Genes \& Development 16 (5): 571-82. doi:10.1101/gad.970702.

Yazlovitskaya, Eugenia M., and Diane L. Persons. 2003. "Inhibition of Cisplatin-Induced ATR Activity and Enhanced Sensitivity to Cisplatin." Anticancer Research 23 (3B): 2275-79.

Zabludoff, Sonya D., Chun Deng, Michael R. Grondine, Adam M. Sheehy, Susan Ashwell, Benjamin L. Caleb, Stephen Green, et al. 2008. "AZD7762, a Novel Checkpoint Kinase Inhibitor, Drives Checkpoint Abrogation and Potentiates DNA-Targeted Therapies." Molecular Cancer Therapeutics 7 (9): 2955-66. doi:10.1158/15357163.MCT-08-0492.

Zhang, Jianming, Priscilla L. Yang, and Nathanael S. Gray. 2009. "Targeting Cancer with Small Molecule Kinase Inhibitors." Nature Reviews Cancer 9 (1): 28-39. doi:10.1038/nrc2559.

Zhang, Y, and Y Xiong. 2001. "A p53 Amino-Terminal Nuclear Export Signal Inhibited by DNA Damage-Induced Phosphorylation." Science (New York, N.Y.) 292 (5523): 1910-15. doi:10.1126/science.1058637.

Zhang, You-Wei, Tony Hunter, and Robert T Abraham. 2006. "Turning the Replication Checkpoint on and off." Cell Cycle (Georgetown, Tex.) 5 (2): 125-28.

Zhao, $\mathrm{H}$, and $\mathrm{H}$ Piwnica-Worms. 2001. "ATR-Mediated Checkpoint Pathways Regulate Phosphorylation and Activation of Human Chk1." Molecular and Cellular Biology 21 (13): 4129-39. doi:10.1128/MCB.21.13.4129-4139.2001.

Zhao, Hui, and Helen Piwnica-Worms. 2001. "ATR-Mediated Checkpoint Pathways Regulate Phosphorylation and Activation of Human Chk1." Molecular and Cellular Biology 21 (13): 4129-39. doi:10.1128/MCB.21.13.4129-4139.2001.

Zhou, Bin-Bing S., and Stephen J. Elledge. 2000. "The DNA Damage Response: Putting Checkpoints in Perspective." Nature 408 (6811): 433-39. doi:10.1038/35044005.

Zou, Lee, David Cortez, and Stephen J. Elledge. 2002. "Regulation of ATR Substrate Selection by Rad17-Dependent Loading of Rad9 Complexes onto Chromatin." Genes \& Development 16 (2): 198-208. doi:10.1101/gad.950302.

Zou, Lee, and Stephen J. Elledge. 2003. "Sensing DNA Damage Through ATRIP Recognition of RPA-ssDNA Complexes." Science 300 (5625): 1542-48. doi:10.1126/science.1083430. 


\section{Appendix}

\section{Efficiency of inhibitors}

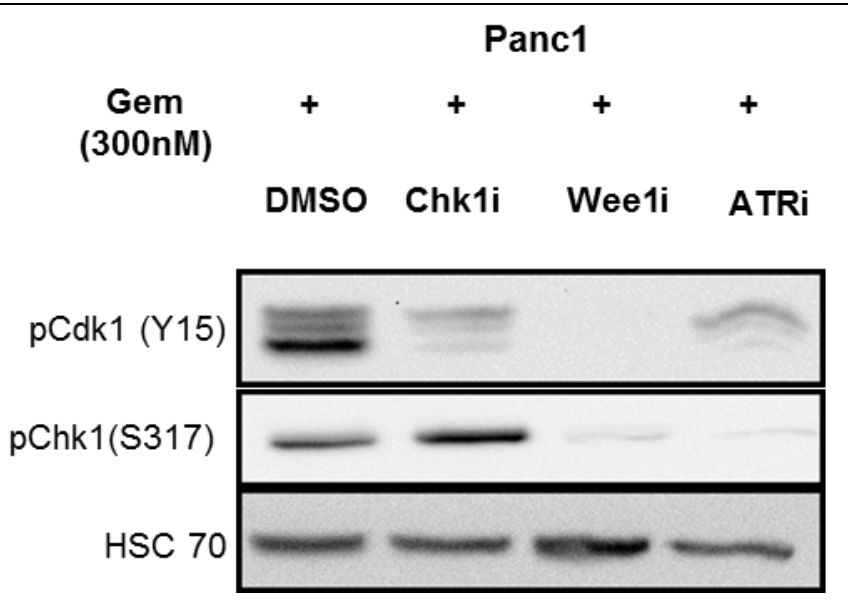

Figure VIII-I Checkpoint kinase inhibitors efficiently inhibit their target kinases.

Panc1 cells were treated with $5 \mu \mathrm{M}$ Chk1i, $1 \mu \mathrm{M}$ Wee1i and $10 \mu \mathrm{M}$ ATRi in the presence of 300nM gemcitabine for $24 \mathrm{~h}$. Blots were stained for the phosphorylation of substrates of each kinaseCdk1 for Wee1, Chk1 and Chk1 for ATR. HSC 70 was stained as loading control. Chk1 controls Cdk activity through phosphorylation of Cdc25 (Sørensen and Syljuåsen 2011).

\section{Knockdown efficiency}

\section{Panc1}

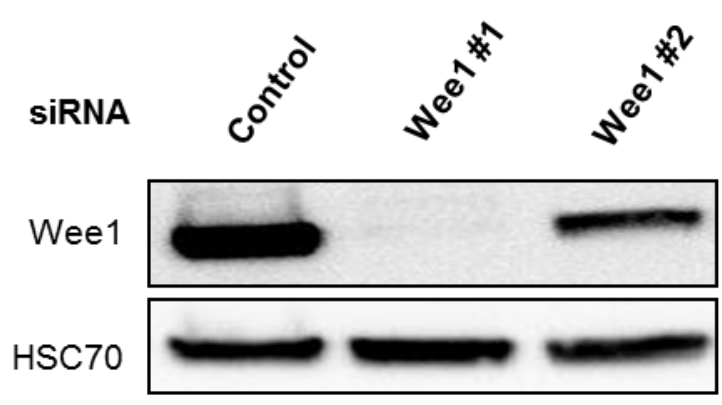

Figure VIII-II Wee1 \#1 siRNA was quite efficient in removing the Wee1 protein.

Panc1 cells were transfected with the negative control no. 1 (Control), Wee1 (Wee1 \#1, \#2) siRNAs and incubated for $48 \mathrm{~h}$. Afterwards, cells were treated with gemcitabine for $24 \mathrm{~h}$ and then harvested. Cell lysate was immunostained for Wee1 and loading control HSC70. 


\section{Panc1}

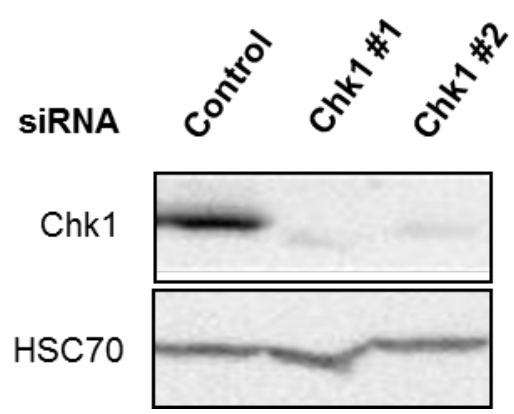

Figure VIII-III Two different siRNAs against Chk1 are efficient in knockdown of Chk1 protein.

Panc1 cells were transfected with the negative control no. 2 (Control), Chk1 (Chk1 \#1, \#2) siRNAs and incubated for $48 \mathrm{~h}$. Afterwards, cells were treated with gemcitabine for $24 \mathrm{~h}$ and then harvested. Cell lysate was immunostained for total levels of Chk1 protein and loading control HSC70.

\section{Double thymidine block}

We observed that inhibition of Wee1 in the absence or presence of gemcitabine increases the premature entry of cells into mitosis. Treatment with Wee1 inhibitor and gemcitabine causes a significant increase in the number of cells with unreplicated DNA in mitosis as early as after $8 \mathrm{~h}$ of treatment.

(a)
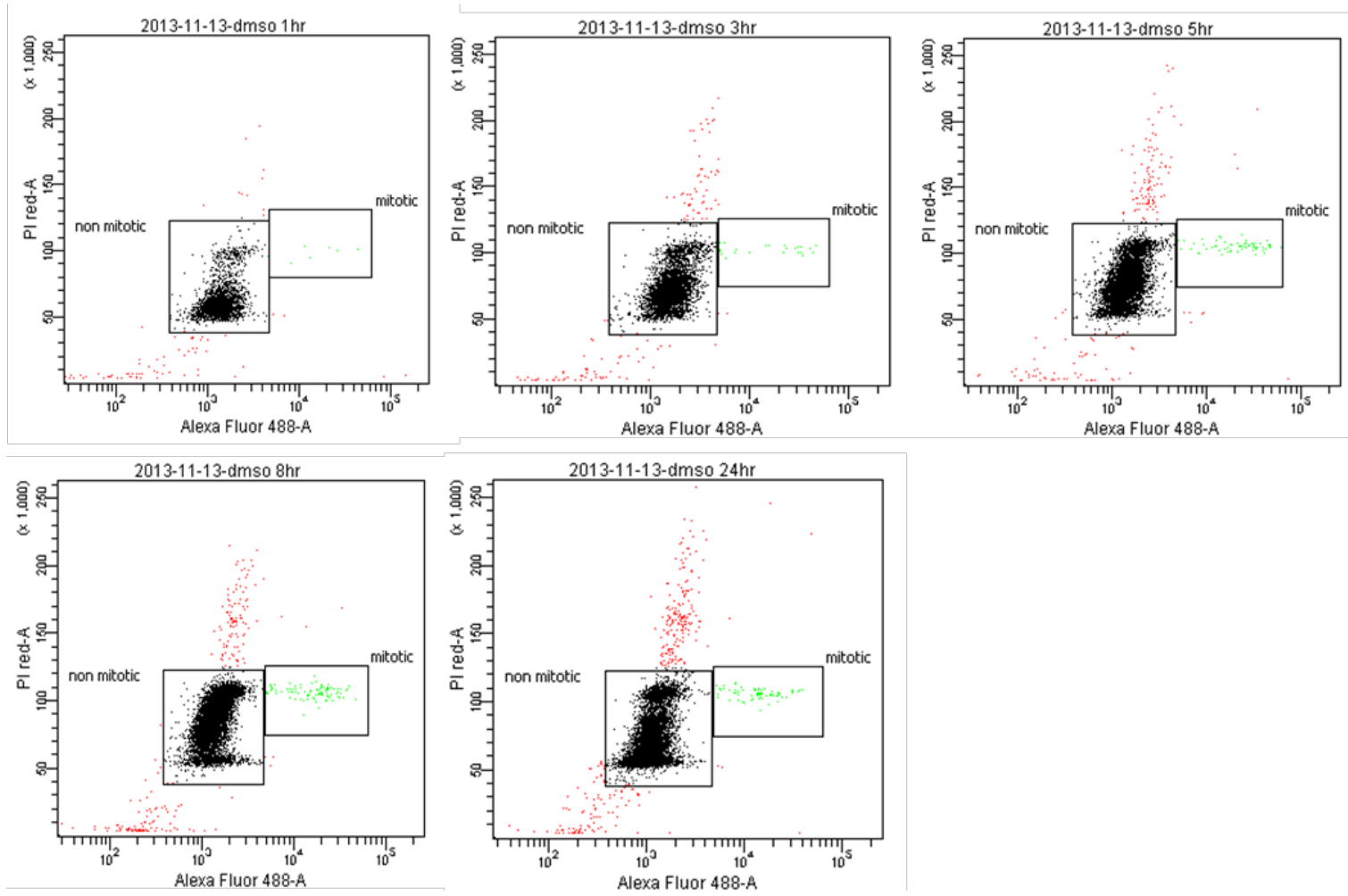
(b)
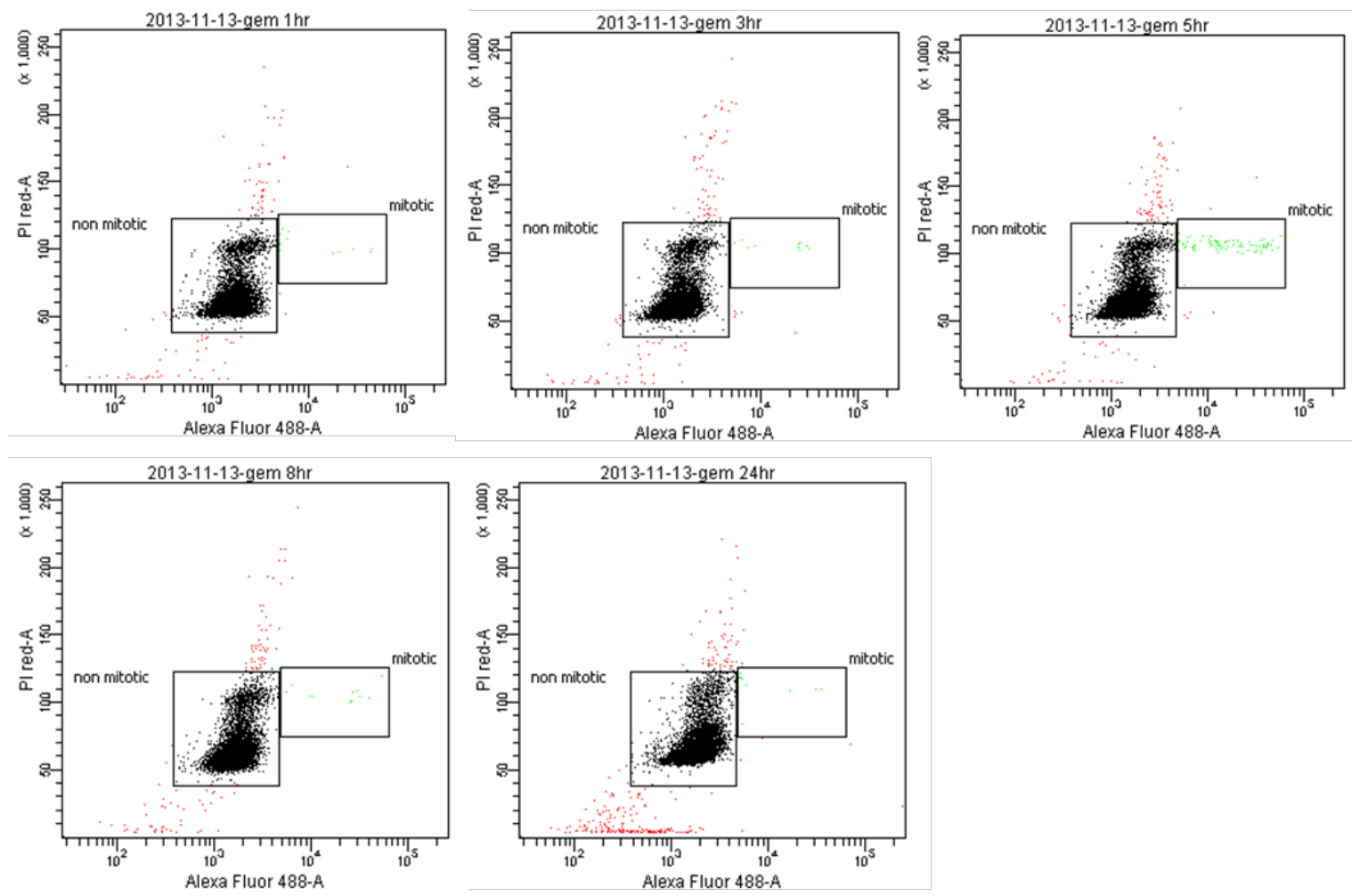

(c)
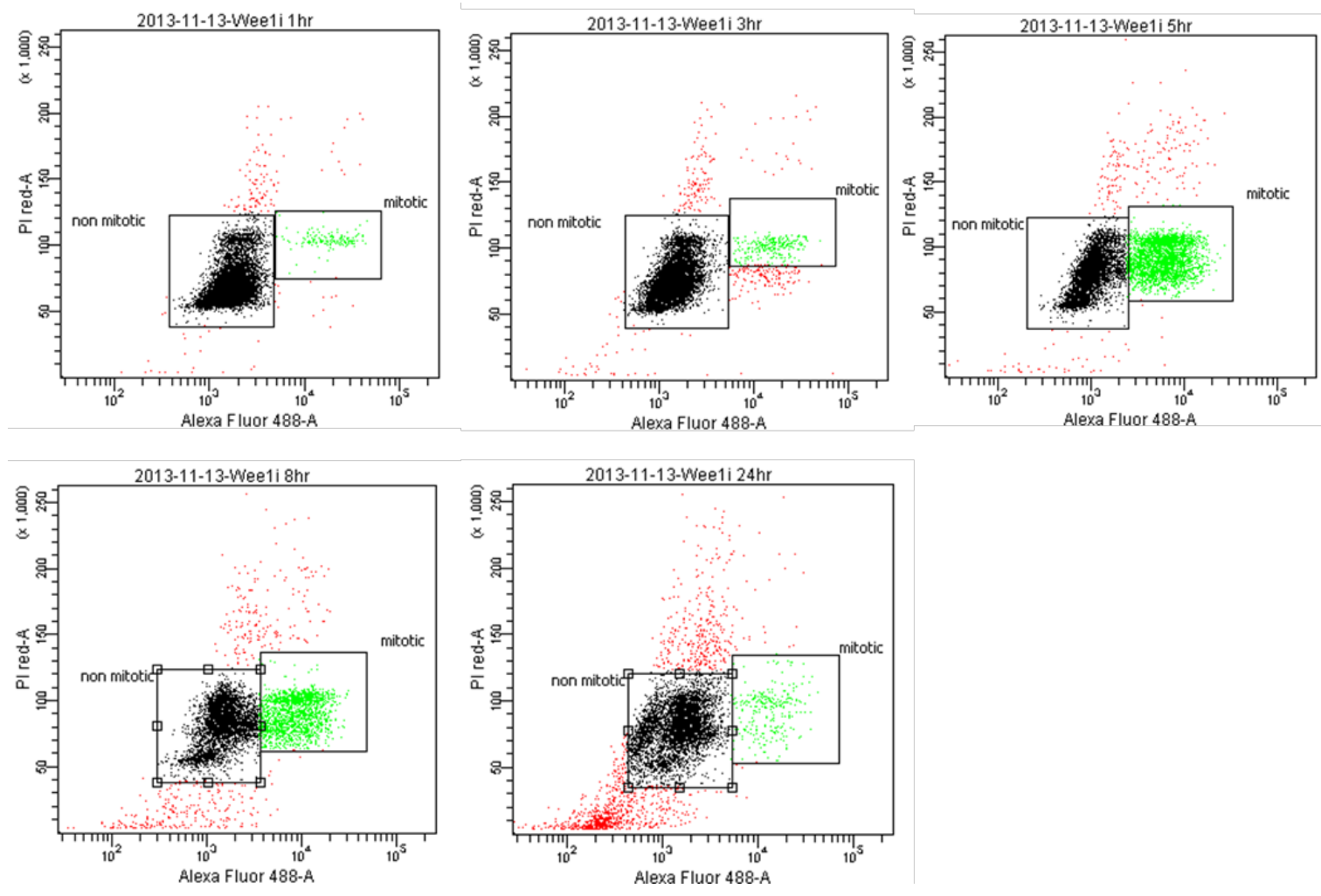
(d)
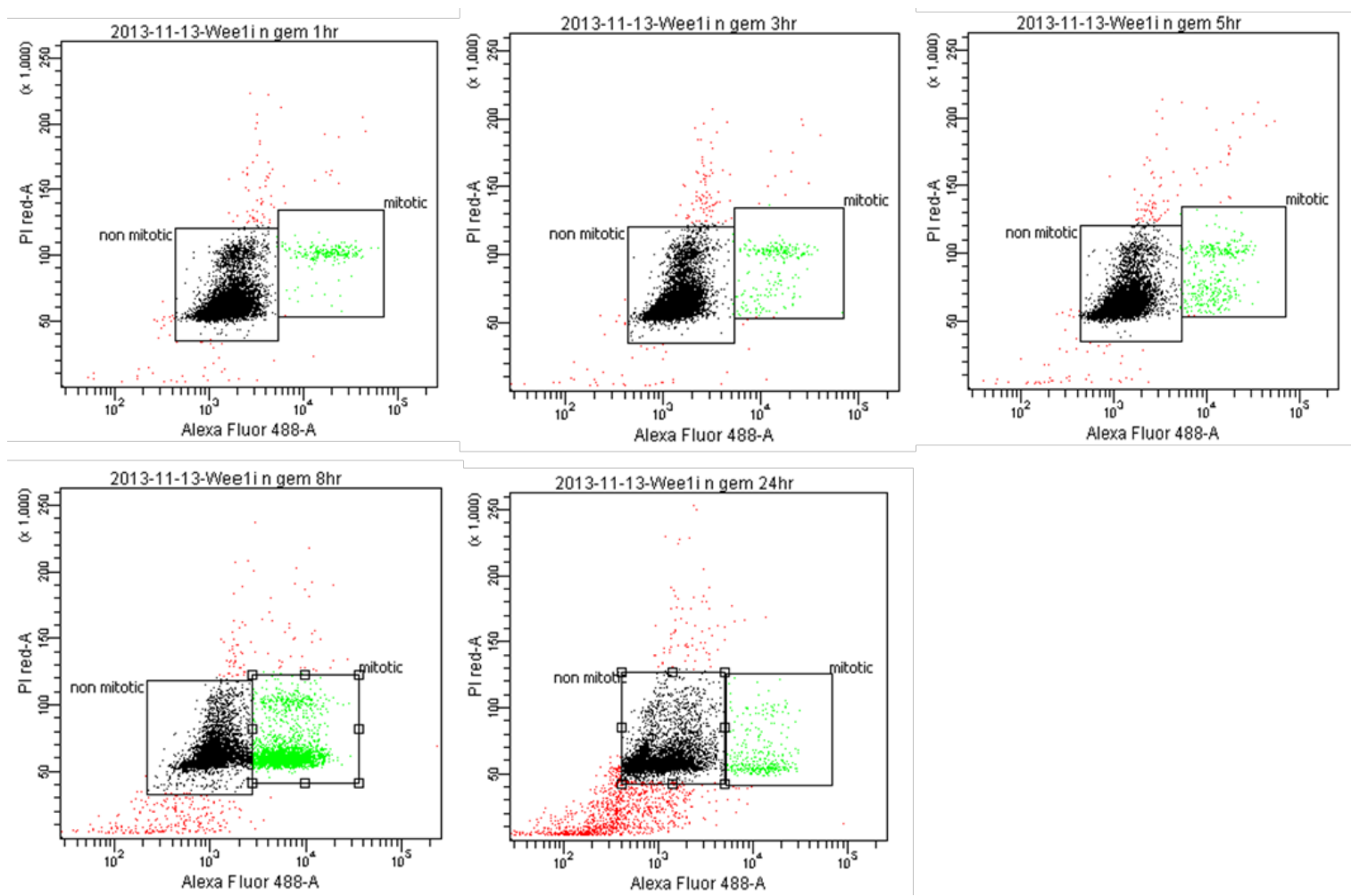

Figure VIII-IV Cells with Wee1 inhibition in the absence or presence of gemcitabine show entry into premature mitosis.

U2OS cells were synchronized using double thymidine block. Afterwards, treatment with gemcitabine or Wee1 inhibitor or a combination of both drugs was done and cells were harvested at different time points. Y-axis represents PI intensity which corresponds to the DNA content of the cells. X-axis represents MPM-2 intensity which corresponds to cells present in mitosis. Green dots represent the cells stained positive for MPM-2 intensity and the dots present near 50 units of PI intensity correspond to the cells entering mitosis prematurely. 